\title{
Duality functors for quantum groupoids
}

\author{
Sophie Chemla and Fabio Gavarini
}

\begin{abstract}
We present a formal algebraic language to deal with quantum deformations of Lie-Rinehart algebras — or Lie algebroids, in a geometrical setting. In particular, extending the ice-breaking ideas introduced by $\mathrm{Xu}$ in [34], we provide suitable notions of "quantum groupoids". For these objects, we detail somewhat in depth the formalism of linear duality; this yields several fundamental antiequivalences among (the categories of) the two basic kinds of "quantum groupoids". On the other hand, we develop a suitable version of a "quantum duality principle" for quantum groupoids, which extends the one for quantum groups — dealing with Hopf algebras — originally introduced by Drinfeld (cf. [8], §7) and later detailed in [12].
\end{abstract}

Mathematics Subject Classification (2010). 17B37, 16T05; 16T15, 17B62, 53D55.

Keywords. Bialgebroids, Lie-Rinehart (bi)algebras, quantum groupoids, quantum duality.

\section{Introduction}

The classical theory of Lie groups, or of algebraic groups, has a quantum counterpart in the theory of "quantum groups". In Drinfeld's language, quantum groups are suitable topological Hopf algebras which are formal deformations either of the algebra of functions on a formal group, or of the universal enveloping algebras of a Lie algebra. These deformations add further structure on the classical object: the formal group inherits a structure of Poisson formal group, and the Lie algebra a structure of Lie bialgebra. Linear duality for topological Hopf algebras reasonably adapts to quantum groups, lifting the analogous duality for their semiclassical limits. On the other hand, Drinfeld revealed a more surprising feature of quantum groups, later named "quantum duality", which somehow lifts the Poisson duality among Poisson (formal) groups. Namely, there exists an equivalence of categories between quantized enveloping algebras and quantized formal groups, which shifts from a quantization of a given Lie bialgebra $L$ to one of the dual Lie bialgebra $L^{*}$.

Another extension of Lie group theory is that of Lie-Rinehart algebras (sometimes loosely called "Lie algebroids"), developed by Rinehart, Huebschmann and others. The notion of Lie-Rinehart algebra $(L,[],, \omega)$ over a commutative ring $A$ lies inbetween $A$-Lie algebras and $k$-Lie algebras of derivations of the form $\operatorname{Der}(A)$. 
Well-known examples come from geometry, such as the global sections of a Lie algebroid, for example the 1-forms over a Poisson manifold (cf. [7], [15], [10]).

The natural algebraic gadgets attached with a Lie-Rinehart algebra are its universal enveloping algebra $V^{\ell}(L)$ and its algebra of jets $J^{r}(L)$, which are in linear duality with each other. Any Lie-Rinehart algebra $L$ can also be seen as a right Lie-Rinehart algebra: thus one can also consider its right enveloping algebra, call it $V^{r}(L)$, anti-isomorphic to $V^{\ell}(L)$, and its dual $J^{\ell}(L)$.

All these algebraic objects - $V^{\ell}(L), V^{r}(L), J^{r}(L)$ and $J^{\ell}(L)$ - are (topological) bialgebroid - left ones when a superscript " $\ell$ " occurs, and right when " $r$ " does. Indeed, they also have an additional property, about their Hopf-Galois map, such that these left/right bialgebroids are actually left or right Hopf left/right bialgebroids an important generalization of Hopf algebras.

Linear duality for (left/right) bialgebroids is twofold: any (left/right) bialgebroid $U$ is naturally a left $A$-module and a right $A$-module, thus one may consider its left dual $U_{*}$ as well as its right dual $U^{*}$. Under mild conditions, $U^{*}$ and $U_{*}$ are naturally (right/left) bialgebroids (see [17]). The $\left(V^{\ell}(L), J^{r}(L)\right)$ is tied together by such a linear duality, and similarly for $\left(V^{r}(L), J^{\ell}(L)\right)$.

When looking for quantizations of Lie-Rinehart algebras, one should consider formal deformations of either $V^{\ell / r}(L)$ or $J^{r / \ell}(L)$, among left/right (topological) bialgebroids: these deformations automatically inherit from their semiclassical limits the additional property of being left/right Hopf left/right bialgebroids. We shall loosely call such deformations "quantum groupoids".

The first step in this direction was made by Ping Xu (cf. [34]): he introduced a notion of quantization of $V^{\ell}(L)$, called quantum universal enveloping algebroid (LQUEAd in short). Then he noticed that any such quantization endows $L$ with a richer structure of Lie-Rinehart bialgebra. This is a direct extension of the notion of Lie bialgebra, in particular, it is a self-dual notion, so if $L$ is a Lie-Rinehart bialgebra then its dual $L^{*}$ is a Lie-Rinehart bialgebra as well (see [20]). Finally, $\mathrm{Xu}$ also provided an example of construction of a non-trivial LQUEAd $\mathcal{D}_{X}[[h]]^{\mathcal{F}}$, by "twisting" the trivial deformation $\mathcal{D}_{X}[[h]]$ of $\mathcal{D}_{X}:=V^{\ell}(\Gamma(T X))$, where $X$ is a Poisson manifold.

The purpose of this paper is to move some further steps in the theory of "quantum groupoids". After recalling some basics of the theory of Lie-Rinehart algebras and bialgebras (Sec. 2), we introduce also some basics of the theory of bialgebroids (Sec. 3): in particular, we dwell on the relevant examples, i.e. universal enveloping algebras and jet spaces for Lie-Rinehart algebras.

We then introduce "quantum groupoids" (Sec. 4). Besides Xu's original notion of LQUEAd, we introduce its right counterpart (in short RQUEAd): a topological right bialgebroid which is a formal deformation of some $V^{r}(L)$. Similarly, we introduce quantizations of jet spaces; a topological right bialgebroid which is a formal deformation of some $J^{r}(L)$ will be called a right quantum formal series 
algebroid (RQFSAd in short); similarly, the left-handed version of this notion gives rise to the definition of left quantum formal series algebroid (LQFSAd in short). Altogether, this gives us four kinds of quantum groupoids; each one of these induces a Lie-Rinehart bialgebra structures on the original Lie-Rinehart algebra one deals with, extending what happens with LQUEAd's.

As a next step, we discuss linear duality for quantum groupoids (Sec. 5). The natural language is that of linear duality for bialgebroids, with some precisions. First, by infinite rank reasons we are lead to consider topological duals. Second, both left and right duals are available, thus taking duals might cause a proliferation of objects. Nevertheless, we can keep this phenomenon under control, so eventually we can bound ourselves to deal with only a handful of duality functors.

In the end, our main result on the subject claims the following: our duality functors provide (well-defined) anti-equivalences between the category of all LQUEAd's and the category of all RQFSAd's (on a same, fixed ring $A_{h}$ ), and similarly also anti-equivalences between the category of all RQUEAd's and the category of all LQFSAd's (on $A_{h}$ again). In addition, if one starts with a given quantum groupoid, which induces a specific (Lie-Rinehart) bialgebra structure on the underlying LieRinehart algebra, then the dual quantization yields the same or the coopposite LieRinehart bialgebra structure - see Theorems 5.1.5 and 5.2.2 for further precisions.

Finally (Sec. 6), we develop a suitable "Quantum Duality Principle" for quantum groupoids. Indeed, we introduce functors "à la Drinfeld", denoted by ()$^{\vee}$ and ( )', which turns (L/R)QFSAd's into (L/R)QUEAd's and viceversa, so to provide an equivalence between the category of LQFSAd's and that of LQUEAd's, and a similar equivalence between RQFSAd's and RQUEAd's. In addition, if one starts with a quantization of some Lie-Rinehart bialgebra $L$, then the (appropriate) Drinfeld's functor gets out of it a quantization of the dual Lie-Rinehart bialgebra $L^{*}$.

For the functor ()$^{\vee}$, Drinfeld's original definition for quantum groups can be easily extended to quantum groupoids. Instead, this is not the case for the functor ()$^{\prime}$ : therefore we have resort to a different characterization (for quantum groups) of it, and adopt that as a definition (for quantum groupoids): this requires linear duality, which sets a strong link with the first part of the paper.

It is worth remarking that linear duality for quantum groupoids interchanges "left" and "right"; instead, quantum duality takes either one to itself: at the end of the day, this means that if one aims to have both linear duality and quantum duality then he/she is forced to deal with all four types of quantum groupoids that we introduced - none of them can be left apart.

At the end (Sec. 7) we present an example, just to illustrate some of our main results on a single — and simple, yet significant enough — toy model.

Acknowledgements. The authors thank Niels Kowalzig for his valuable comments and hints. 


\section{Lie-Rinehart algebras and bialgebras}

Throughout this paper, $k$ will be a field and $A$ will be a unital, associative $k-$ algebra; we assume $k$ to have characteristic zero (though for most definitions and constructions this is not necessary). Moreover, for all objects defined in this section we assume in addition that $A$ is also commutative.

2.1. Lie-Rinehart algebras. To begin with, we introduce the notion of (left) LieRinehart algebra ( or "Lie algebroid").

Definition 2.1.1. A (left) Lie-Rinehart algebra (see [30]) is a triple $(A, L, \omega)$ where: $L$ is a $k$-Lie algebra, $L$ is an $A$-module, and $\omega$ is an $A$-linear morphism of Lie $k$-algebras from $L$ to $\operatorname{Der}(A)$, called anchor (map), such that the following compatibility relation holds:

$$
\forall D, D^{\prime} \in L, \forall f \in A, \quad\left[D, f D^{\prime}\right]=\omega(D)(f) D^{\prime}+f\left[D, D^{\prime}\right]
$$

In particular, if $L$ is finitely generated projective as an $A$-module, then $(A, L, \omega)$ will be called a finite projective Lie-Rinehart algebra.

Notation. When there is no ambiguity, the Lie-Rinehart algebra $(A, L, \omega)$ will be written $L$.

Examples 2.1.2. Any Lie algebra over $A$ is a Lie-Rinehart algebra whose anchor map $\omega$ is 0 (and conversely). On the other hand, $(A, \operatorname{Der}(A), i d)$ is a Lie-Rinehart algebra too. Another example is the $A$-module $D_{A}$ of Kähler differentials on any Poisson algebra $A$ (see [15]).

In the setup of differential geometry, natural examples of Lie-Rinehart algebras arise as spaces of global sections of Lie algebroids; for instance, such an example (of Lie algebroid) is given by the vector bundle $\Omega_{P}^{1}$ of differential forms of degree 1 on a Poisson manifold $P$ (see, e.g., [7]).

2.1.3. Differentials for Lie-Rinehart algebras. Given a finite projective LieRinehart algebra $(A, L, \omega)$, it is known that $\bigwedge_{A} L^{*}=\oplus_{n} \bigwedge_{A}^{n} L^{*}$ admits a differential $d_{L}$ that makes it into a differential algebra. Here $d_{L}: \bigwedge_{A}^{n} L^{*} \longrightarrow$ $\bigwedge_{A}^{n+1} L^{*}$ is defined as follows: for all $\lambda \in \bigwedge_{A}^{n} L^{*}$ and for all $\left(X_{1}, X_{2}, \ldots, X_{n+1}\right) \in$ $L^{n+1}$, one has

$$
\begin{aligned}
\left(d_{L} \lambda\right)\left(X_{1}, \ldots, X_{n+1}\right) & =\sum_{i=1}^{n+1}(-1)^{i+1} \omega\left(X_{i}\right)\left(\lambda\left(X_{1}, \ldots, \widehat{X_{i}}, \ldots, X_{n+1}\right)\right. \\
& +\sum_{i<j}(-1)^{i+j} \lambda\left(\left[X_{i}, X_{j}\right], X_{1}, \ldots, \widehat{X_{i}}, \ldots, \widehat{X}_{j}, \ldots, X_{n+1}\right)
\end{aligned}
$$

In the case where $L=T X$, the differential $d_{L}$ coincides with the de Rham differential. 
Definition 2.1.4. Let $(A, L, \omega)$ be a (left) Lie-Rinehart algebra. The (left) universal enveloping algebra of $L$ is the $k$-algebra $V^{\ell}(L):=T_{k}^{+}(A \oplus L) / I$ where $T_{k}^{+}(A \oplus L)$ is the positive part of the tensor $k$-algebra over $A \oplus L$ and $I$ is the two-sided ideal in $T_{k}^{+}(A \oplus L)$ generated by the elements

$$
\begin{array}{r}
a \otimes b-a b, a \otimes \xi-a \xi, \quad \xi \otimes \eta-\eta \otimes \xi-[\xi, \eta], \quad \xi \otimes a-a \otimes \xi-\omega(\xi)(a) \\
\forall a, b \in A, \xi, \eta \in L .
\end{array}
$$

Remarks 2.1.5. (a) Note that $V^{\ell}(L)$ is a filtered ring, its (increasing) filtration $\left\{V_{n}^{\ell}(L)\right\}_{n \in \mathbb{N}}$ being defined by $V_{0}^{\ell}(L):=A, V_{n+1}^{\ell}(L):=V_{n}^{\ell}(L)+V_{n}^{\ell}(L) \cdot L(n \in \mathbb{N})$. We denote by $\operatorname{Gr}\left(V^{\ell}(L)\right)$ the associated graded algebra. It is known (cf. [30]) that if $L$ is projective as an $A$-module, then $\operatorname{Gr}\left(V^{\ell}(L)\right) \cong S_{A}(L)$. Moreover, $\iota_{A}: A \longrightarrow V^{\ell}(L)$ and $\iota_{L}: L \longrightarrow V^{\ell}(L)$ are monomorphisms.

(b) The Lie-Rinehart algebras $L=(L, A,[],, \omega)$ and $L^{o p}:=(L, A,-[],,-\omega)$ are isomorphic via the isomorphims $F$ defined by $F(D):=-D$ for all $D \in L$ and $F(a):=a$ for all $a \in A$.

(c) If $X$ is a (smooth) manifold and $A=\mathcal{C}^{\infty}(X)$, then $V^{\ell}(\operatorname{Der}(A))$ for the LieRinehart algebra $(A, \operatorname{Der}(A), i d)$ is the $k$-algebra of global differential operators on $X$.

2.1.6. From a finite projective Lie-Rinehart algebra to a free Lie-Rinehart algebra. Most of the time, we will work with finite projective Lie-Rinehart algebras. This is a reasonable hypothesis as Lie-Rinehart algebras coming from the geometry are finite projective. Several times in this article, we will prove results for (finite) free Lie-Rinehart algebra and then extend them to finite projective Lie-Rinehart algebras. We now explain the key step for this.

Let $L$ be a finite projective Lie-Rinehart algebra. There exist a finite projective $A$-module $Q$ such that $F=L \oplus Q$ is a finite rank free $A$-module. We can endow $F$ with the following Lie-Rinehart algebra structure: for all $D, D_{1}, D_{2} \in L$, $E, E_{1}, E_{2} \in Q$, we set

$$
\omega_{F}(D+E):=\omega_{L}(D), \quad\left[D_{1}+E_{1}, D_{2}+E_{2}\right]:=\left[D_{1}, D_{2}\right]
$$

that is, the structure of $L$ is extended trivially to $F=L \oplus Q$. Then $V^{\ell}(F)=$ $V^{\ell}(L) \otimes_{A} S(Q)$.

The $A$-module $L_{Q}:=L \oplus Q \oplus L \oplus Q \oplus \cdots=F \oplus F \oplus F \oplus \cdots$ is a free $A$-module. Define $R=Q \oplus L \oplus Q \oplus L \oplus \cdots$; then $R$ is a free $A$-module such that $L_{Q}=L \oplus R$ is a free $A$-module (cf. [14]). We set on $L_{Q}$ the Lie-Rinehart algebra structure (for all $D, D_{1}, D_{2} \in L, B, B_{1}, B_{2} \in R$ )

$$
\omega_{L_{Q}}(D+B):=\omega_{L}(D), \quad\left[D_{1}+B_{1}, D_{2}+B_{2}\right]:=\left[D_{1}, D_{2}\right]
$$

in other words, the Lie-Rinehart algebra structure of $L$ is extended trivially to $L_{Q}=L \oplus R$. 
Definition 2.1.7. Let an $A$-basis $\left\{b_{1}, \ldots, b_{n}\right\}$ of $F$ be given. Then one can construct a basis $\left\{v_{t}\right\}_{t \in T}$ of $R$ and an $A$-basis $\left\{e_{t}\right\}_{t \in T}$ of $L_{Q}$ both indexed by $T:=\mathbb{N} \times$ $\{1, \ldots, n\}$. Any such basis for $L_{Q}$ will be called a good basis. For later use, if $i=\left(i_{1}, i_{2}\right) \in T$ we set $\varpi\left(e_{i}\right):=i_{1}$.

We set $Y=\oplus_{i=1}^{n} k b_{i}$ and $Z=\oplus_{t \in T} k v_{t} \simeq Y \oplus Y \oplus Y \cdots$ so that $F=A \otimes_{k} Y$ and $R=A \otimes_{k} Z$. We have then $V^{\ell}\left(L_{Q}\right)=V^{\ell}(L) \otimes_{k} S(Z)$.

2.1.8. Right Lie-Rinehart algebras. For the sake of completeness, we have to mention that one can also, in a symmetric way, consider the notion of right LieRinehart algebra, as follows:

Definition 2.1.9. A right Lie-Rinehart algebra is a triple $(A, L, \omega)$ where $L$ is a $k-$ Lie algebra, $L$ is a right $A$-module, and $\omega$ is an $A$-linear morphism of Lie $k$-algebras from $L$ to $\operatorname{Der}(A)$, called anchor (map), such that the following compatibility relation holds:

$$
\forall D, D^{\prime} \in L, \quad \forall f \in A, \quad\left[D, D^{\prime} \cdot f\right]=D^{\prime} \cdot \omega(D)(f)+\left[D, D^{\prime}\right] \cdot f
$$

Remark 2.1.10. As $A$ is commutative, a Lie-Rinehart algebra can be considered as a right Lie-Rinehart algebra and viceversa. However, the enveloping algebra defined by the notion of right Lie-Rinehart algebra is different from that defined by a (left) Lie-Rinehart algebra.

Definition 2.1.11. Let $(A, L, \omega)$ be a right Lie-Rinehart algebra. The (right) universal enveloping algebra of $L$ is the $k$-algebra $V^{r}(L):=T_{k}^{+}(A \oplus L) / I$ where $T_{k}^{+}(A \oplus L)$ is the positive part of the tensor $k$-algebra over $A \oplus L$ and $I$ is the two-sided ideal in $T_{k}^{+}(A \oplus L)$ generated by the elements

$$
\begin{aligned}
a \otimes b-a b, \quad \xi \otimes a-\xi \cdot a, \quad \xi \otimes \eta-\eta \otimes \xi-[\xi, \eta], & \xi \otimes a-a \otimes \xi-\omega(\xi)(a) \\
& \forall a, b \in A, \xi, \eta \in L .
\end{aligned}
$$

Next result clarifies the link between left and right enveloping algebras of a single Lie-Rinehart algebra $L$. Hereafter, $L^{o p}$ denotes the "opposite" Lie-Rinehart algebra to $L-$ cf. Remarks 2.1.5 - while $\mathfrak{A}^{o p}$ denotes the opposite of any (associative) algebra $\mathfrak{A}$.

Proposition 2.1.12. For any Lie-Rinehart algebra $L$, the algebras $V^{r}(L)^{o p}$ and $V^{\ell}\left(L^{o p}\right)$ are equal, and there is an algebra isomorphism $\Xi: V^{\ell}(L) \longrightarrow V^{r}(L)^{\mathrm{op}}$, $a \mapsto a, D \mapsto-D$ (for all $a \in A, D \in L)$. 
2.2. Lie-Rinehart bialgebras. We are now ready to introduce the notion of LieRinehart bialgebra (cf. [26], [20], [16]).

Definition 2.2.1. A Lie-Rinehart bialgebra is a pair $\left(L_{1}, L_{2}\right)$ of finitely generated projective $A$-modules in duality — that is, $L_{1} \cong L_{2}^{*}$ and $L_{2} \cong L_{1}^{*}$ - each of them being endowed with Lie-Rinehart algebra structures such that the differential $d_{1}$ on $\bigwedge_{A} L_{1}$ arising from the Lie-Rinehart structure on $L_{2} \cong L_{1}^{*}$ is a derivation of the Lie bracket of $L_{1}$, that is

$$
d_{1}([X, Y])=\left[d_{1}(X), Y\right]+\left[X, d_{1}(Y)\right] \quad \text { for all } X, Y \in L_{1} .
$$

In general, if $L$ is a finitely generated projective $A$-module, then its linear dual $L^{*}$ (as an $A$-module) is finitely generated projective as well: in this case, in the following we shall say that " $L$ is a Lie-Rinehart bialgebra" to mean that $\left(L, L^{*}\right)$ has a structure of Lie-Rinehart bialgebra, and we shall denote the differential of $\bigwedge_{A} L$ mentioned above by $d_{L^{*}}$ or $\delta_{L}$.

Remarks 2.2.2. (a) The conditions of Definition 2.2.1 do not change if one switches $L_{1}$ and $L_{2}$ (cf. [20]).

(b) It follows from the definition that the differential $\delta_{L}$ of $L$ in a Lie-Rinehart bialgebra $\left(L, L^{*}\right)$ is uniquely determined by its restriction to $A$ and $L$ - the degree 0 and degree 1 pieces of $\bigwedge_{A} L$.

(c) If $\left(L, L^{*}\right)$ is a Lie-Rinehart bialgebra, we can read off the explicit relation between the Lie-Rinehart structure of $L^{*}$ - its anchor map $\omega_{L^{*}}$ and its Lie bracket $[,]_{L^{*}}$ - and the differential $\delta_{L}$ of $L$ as follows: if $D^{*}, E^{*} \in L^{*}, X \in L, a \in A$, then

$$
\begin{aligned}
\omega_{L^{*}}\left(D^{*}\right)(a) & =\left\langle\delta_{L}(a), D^{*}\right\rangle, \\
\left\langle X,\left[D^{*}, E^{*}\right]_{L^{*}}\right\rangle+\left\langle\delta_{L}(X), D^{*} \wedge E^{*}\right\rangle & =\omega_{L^{*}}\left(D^{*}\right)\left(\left\langle X, E^{*}\right\rangle\right)-\omega_{L^{*}}\left(E^{*}\right)\left(\left\langle X, D^{*}\right\rangle\right),
\end{aligned}
$$

where $\langle$,$\rangle denotes the natural pairing between L$ and $L^{*}$. Indeed, one can use these formulas either to deduce $\omega_{L^{*}}$ and $[,]_{L^{*}}$ from $\delta_{L}$, or to deduce the latter from $\omega_{L^{*}}$ and $[,]_{L^{*}}$

(d) Let $\left(L, L^{*}\right)$ be a Lie-Rinehart bialgebra. Denote by $d$ the differential on $\bigwedge_{A} L^{*}$ arising from the Lie-Rinehart structure on $L$ and $d_{*}\left(=\delta_{L}\right)$ the differential on $\bigwedge_{A} L$ coming from the Lie-Rinehart structure on $L^{*}$. Then $A$ inherits a Poisson algebra structure by $\{f, g\}:=\left\langle d f, d_{*} g\right\rangle$ for all $f, g \in A$. (see [20], [33]); moreover, one has $[d f, d g]=d\{f, g\}$ and $d_{*}\{f, g\}=-\left[d_{*} f, d_{*} g\right]$.

(e) If $\left(L, L^{*}\right)$ is a Lie-Rinehart bialgebra, then $\left(L^{o p}, L^{*}\right),\left(L,\left(L^{*}\right)^{o p}\right)$ and $\left(L^{o p},\left(L^{*}\right)^{o p}\right)$ are Lie-Rinehart bialgebras too. Identifying any Lie-Rinehart bialgebra, as a pair, with the left-hand of the pair, say $L \equiv\left(L, L^{*}\right)$, we write $L^{o p} \equiv\left(L^{o p}, L^{*}\right)$ - the "opposite" to $L ;\left(L,\left(L^{*}\right)^{o p}\right) \equiv L_{\text {coop }}$ - the "coopposite"; and $L_{\text {coop }}^{o p} \equiv\left(L^{o p},\left(L^{*}\right)^{o p}\right)$ 一 the "opposite-coopposite".

(f) By means of the so called r-matrices one can introduce the class of coboundary Lie-Rinehart bialgebras, and among them the triangular ones: we refer the interested readers to [34]. 


\section{Left and right bialgebroids}

Let again $k$ be a field, and $A$ a unital, associative $k$-algebra. We define $A^{e}:=A \otimes_{k} A^{o p}$.

3.1. A-rings, $A$-corings. We begin this section introducing the notions of $A-$ ring and $A$-coring, which are direct generalizations of the notions of algebra and coalgebra over a commutative ring.

Definition 3.1.1. Let $A$ be a $k$-algebra as above. An $A$-ring is a triple $\left(H, m_{H}, \iota\right)$ where $H$ is an $A^{e}$-module, $m_{H}: H \otimes_{A} H \longrightarrow H$ and $\iota: A \longrightarrow H$ are $A^{e}$-module morphisms such that

$m_{H} \circ\left(m_{H} \otimes i d_{H}\right)=m_{H} \circ\left(i d_{H} \otimes m_{H}\right), \quad m_{H} \circ\left(\iota \otimes i d_{H}\right)=i d_{H}=m_{H} \circ\left(i d_{H} \otimes \iota\right)$

where in the second set of identities we make the identifications $H \otimes_{A} A \cong H$ and $A \otimes_{A} H \cong H$.

It is well known (see [4]) that $A$-rings $H$ correspond bijectively to $k$-algebra homomorphisms $\iota: A \longrightarrow H$. With this characterization, the $A^{e}$-module structure on $H$ can be expressed as follows: $a \cdot h \cdot b=\iota(a) h \iota(b)$ for $a, b \in A, h \in H$. The dual notion (" $A$-coring") is the following:

Definition 3.1.2. An $A$-coring is a triple $(C, \Delta, \epsilon)$ where $C$ is an $A^{e}$-module (with left action $L_{A}$ and right action $R_{A}$ ), $\Delta: C \longrightarrow C \otimes_{A} C$ and $\epsilon: C \longrightarrow A$ are $A^{e}$-module morphisms such that

$\left(\Delta \otimes i d_{C}\right) \circ \Delta=\left(i d_{C} \otimes \Delta\right) \circ \Delta, \quad L_{A} \circ\left(\epsilon \otimes i d_{C}\right) \circ \Delta=i d_{C}=R_{A} \circ\left(i d_{C} \otimes \epsilon\right) \circ \Delta$

As usual, we adopt Sweedler's $\Sigma$-notation $\Delta(c)=c_{(1)} \otimes c_{(2)}$ or $\Delta(c)=c^{(1)} \otimes$ $c^{(2)}$ for $c \in C$.

Let $A$ be as above, and consider now $A^{e}$ as base $k$-algebra. An $A^{e}$-ring $H$ can be described by a $k$-algebra morphism $\iota: A^{e} \longrightarrow H$. Let us consider its restrictions $s^{\ell}:=\iota\left(-\otimes_{k} 1_{H}\right): A \longrightarrow H, t^{\ell}:=\iota\left(1_{H} \otimes_{k}-\right): A \longrightarrow H$, which are called respectively source and target maps. Thus an $A^{e}$-ring $H$ carries two (left) $A$ module structures and two (right) $A^{o p}$-module structures: for all $a, a^{\prime} \in A, h \in H$, we write

$$
a \triangleright h \triangleleft \tilde{a}:=s^{\ell}(a) t^{\ell}(\tilde{a}) h, \quad a \triangleright h \triangleleft \tilde{a}:=h t^{\ell}(a) s^{\ell}(\tilde{a})
$$

As usual, the tensor product of $H$ with itself (as an $A$-bimodule, i.e. an $A^{e}$-module) is defined as

$$
H_{\triangleleft} \otimes_{A} H:=H \otimes_{k} H /\left\{(u \triangleleft a) \otimes u^{\prime}-u \otimes\left(a \triangleright u^{\prime}\right)\right\}_{a \in A, u, u^{\prime} \in H}
$$

Now we define the left Takeuchi product (of the $A^{e}$-ring $H$ with itself) $H_{\triangleleft} \times_{\triangleright} H \subseteq H_{\triangleleft} \otimes_{\triangleright} H$

$H_{\triangleleft} \times_{\triangleright} H:=\left\{\sum_{i} u_{i} \otimes u_{i}^{\prime} \in H_{\triangleleft} \otimes_{\triangleright} H \mid \sum_{i}\left(a \triangleright u_{i}\right) \otimes_{A} u_{i}^{\prime}=\sum_{i} u_{i} \otimes\left(u_{i}^{\prime} \triangleleft a\right), \forall a \in A\right\}$ 
By construction, $H_{\triangleleft} \times_{\triangleright} H$ has a natural structure of $A^{e}$-module, induced by that of $H_{\triangleleft} \otimes_{A} H$. Even more, $H_{\triangleleft} \times_{A} H$ is an $A^{e}$-ring, via factorwise multiplication, with unit element $1_{H} \otimes 1_{H}$ and with $\iota_{H_{\triangleleft} \times{ }^{\triangleright}}(a \otimes \tilde{a}):=s^{\ell}(a) \otimes t^{\ell}(\tilde{a})$. Note that this instead is not the case for $H_{\triangleleft} \otimes_{A} H$.

3.2. Left bialgebroids. We introduce now the notion of left bialgebroid, as well as some related items (see [32], [25], [34], [4] and [22], Chapter 2, for a detailed history of this notion). We begin with the very definition:

Definition 3.2.1. A left $A$-bialgebroid is a $k$-module $H$ that carries simultaneously a structure of an $A^{e}$-ring $\left(H, s^{\ell}, t^{\ell}\right)$ and of an $A$-coring $\left(H, \Delta_{\ell}, \epsilon\right)$ subject to the following compatibility relations:

(i) The $A^{e}$-module structure on the $A$-coring $\left(H, \Delta_{\ell}, \epsilon\right)$ is that of $\triangleright H_{\triangleleft}$, namely (for all $a, \tilde{a} \in A, h \in H) a \triangleright h \triangleleft \tilde{a}:=s^{\ell}(a) t^{\ell}(\tilde{a}) h$.

(ii) The coproduct map $\Delta^{\ell}$ is a unital $k$-algebra morphism taking values in $H_{\triangleleft} \times_{\triangleright} H$

(iii) The (left) counit map $\epsilon$ has the following property: for $a, \tilde{a} \in A, u, u^{\prime} \in H$, one has

$$
\begin{gathered}
\epsilon\left(s^{\ell}(a) t^{\ell}(\tilde{a}) u\right)=a \epsilon(u) \tilde{a}, \\
\epsilon\left(u u^{\prime}\right)=\epsilon\left(u s^{\ell}\left(\epsilon\left(u^{\prime}\right)\right)\right)=\epsilon\left(u t^{\ell}\left(\epsilon\left(u^{\prime}\right)\right)\right), \\
\epsilon(1)=1 .
\end{gathered}
$$

Remarks 3.2.2. A left bialgebroid $H$ over $A$ has the following properties (for $a \in A$, $u \in H)$ :

(a) $\Delta_{\ell}(a \triangleright u \triangleleft \tilde{a})=\left(a \triangleright u_{(1)}\right) \otimes\left(u_{(2)} \triangleleft \tilde{a}\right)$,

$\Delta_{\ell}(a \triangleright u \triangleleft \tilde{a})=\left(u_{(1)} \triangleleft \tilde{a}\right) \otimes\left(a \triangleright u_{(2)}\right)$

(using $\Sigma$-notation $\Delta(u)=u_{(1)} \otimes u_{(2)}$ as usual, cf. Definition 3.1.2)

(b) $H$ acts on $A$ on the left (cf. [22]) by $u . a:=\epsilon\left(u s^{\ell}(a)\right)=\epsilon\left(u t^{\ell}(a)\right)$; we shall also use the notation $u(a):=u \cdot a$, and call this the left anchor of the left bialgebroid $H$ (cf. [34]).

(c) $t^{\ell}(\epsilon(x)) \otimes 1=t^{\ell}\left(\epsilon\left(x_{(1)}\right)\right) \otimes s^{\ell}\left(\epsilon\left(x_{(2)}\right)\right)=1 \otimes s^{\ell}(\epsilon(x)) \quad$ for all $x \in H$.

(d) as a matter of notation, if $\left(H, A, s^{\ell}, t^{\ell}, \Delta, \epsilon\right)$ is a left bialgebroid, we set $H^{+}:=\operatorname{Ker}(\epsilon)$.

Definition 3.2.3. Let $\mathcal{H}=\left(H, A, s^{\ell}, t^{\ell}, \Delta, \epsilon\right)$ and $\hat{\mathcal{H}}=\left(\hat{H}, \hat{A}, \hat{s}^{\ell}, \hat{t}^{\ell}, \hat{\Delta}, \hat{\epsilon}\right)$ be two left bialgebroids. A morphism of left bialgebroids from $\mathcal{H}$ to $\hat{\mathcal{H}}$ is a pair $(f, F)$ 
where $f: A \longrightarrow \hat{A}$ is a morphism of algebras, $F: H \longrightarrow \hat{H}$ is a morphism of rings and of corings, and $F \circ s^{\ell}=\hat{s}^{\ell} \circ f, F \circ t^{\ell}=\hat{t}^{\ell} \circ f$.

We denote by (LBialg) the category of left bialgebroids, whose objects are left bialgebroids and morphisms are defined as above. Inside it, $\left(\operatorname{LBialg}_{A}\right)$ is the subcategory whose objects are all the left bialgebroids over $A$, and whose morphisms are all the morphisms in (LBialg) of the form (id, $F)$.

3.2.4. Twistors of left bialgebroids. Let $H$ be a left bialgebroid. Given $\mathcal{F}=$ $\sum_{i} x_{i} \otimes y_{i} \in H_{\triangleleft} \otimes_{\triangleright} H$ (with $x_{i}, y_{i} \in H$ ), define $s_{\mathcal{F}}^{\ell}: A \longrightarrow H$ by $s_{\mathcal{F}}^{\ell}(a):=$ $\sum_{i} s^{\ell}\left(x_{i}(a)\right) y_{i}$ and $t_{\mathcal{F}}^{\ell}: A \longrightarrow H$ by $t_{\mathcal{F}}^{\ell}(a)=\sum_{i} t^{\ell}\left(y_{i}(a)\right) x_{i}$. Moreover, for any $a, b \in A$ set $a *_{\mathcal{F}} b:=s_{\mathcal{F}}^{\ell}(a)(b)=\sum_{i} x_{i}(a) y_{i}(b)$.

Proposition 3.2.5. (cf. [34]) Assume that $\mathcal{F} \in \underset{A}{H \otimes H}$ satisfies the following conditions:

(i) $(\Delta \otimes i d)(\mathcal{F}) \cdot \mathcal{F}_{1,2}=(i d \otimes \Delta)(\mathcal{F}) \cdot \mathcal{F}_{2,3} \quad$ inside $H \otimes_{A} H \otimes_{A} H$

(ii) $m((\epsilon \otimes i d)(\mathcal{F}))=1_{H}, \quad m((i d \otimes \epsilon) \mathcal{F})=1_{H}$ where $\mathcal{F}_{1,2}=\mathcal{F} \otimes 1_{H} \in H \otimes_{A} H \otimes_{A} H$ and $\mathcal{F}_{2,3}=1_{H} \otimes \mathcal{F} \in H \otimes_{A} H \otimes_{A} H$.

Then one has $\mathcal{F} \cdot\left(t_{\mathcal{F}}^{\ell}(a) \otimes 1_{H}-1_{H} \otimes s_{\mathcal{F}}^{\ell}(a)\right)=0$ inside $H \otimes_{A} H$ (for all $a \in A$ ). Moreover, if $\mathcal{F}$ satisfies (i) and (ii) above, then

(a) $\left(A, *_{\mathcal{F}}\right)$ is an associative algebra, denoted $A_{\mathcal{F}}$, and $a *_{\mathcal{F}} 1=a=1 *_{\mathcal{F}} a$ for all $a \in A$;

(b) $s_{\mathcal{F}}^{\ell}: A_{\mathcal{F}} \longrightarrow H$ is an algebra morphism and $t_{\mathcal{F}}^{\ell}: A_{\mathcal{F}} \longrightarrow H$ is an algebra antimorphism.

Now let $M$ be a module over $H$ (as an algebra): then $M$ has also a natural $A^{e}$ module structure. If $\mathcal{F}$ is a twistor of $H$, then $M$ has also a natural $A_{\mathcal{F}}^{e}$-module structure. Consequently, if $M_{1}$ and $M_{2}$ are two $H$-modules, the tensor products $M_{1 \triangleleft} \otimes_{\triangleright} M_{2}$ and $M_{1 \triangleleft}{\underset{A \mathcal{F}}{\mathcal{F}}}_{\triangleright} M_{2}$ are well defined.

Corollary 3.2.6. (cf. [34]) Let $M_{1}$ and $M_{2}$ be two left H-modules. Then there exists a well defined k-linear map $\mathcal{F}^{\#}: M_{1 \triangleleft} \bigotimes_{A_{\mathfrak{F}}} M_{2} \longrightarrow M_{1 \triangleleft} \bigotimes_{A} M_{2}$ given by $m_{1} \otimes m_{2} \mapsto \mathcal{F} \cdot\left(m_{1} \otimes m_{2}\right)$.

We say that $\mathcal{F}$ is invertible if $\mathcal{F}^{\#}$ is a $k$-vector space isomorphism for any choice of $M_{1}$ and $M_{2}$. In this case, taking $M_{1}=M_{2}=H$ we get a $k$-linear isomorphism $\mathcal{F}^{\#}: H \otimes_{A_{\mathcal{F}}} H \longrightarrow H \otimes_{A} H$.

Definition 3.2.7. An element $\mathcal{F} \in H \otimes H$ is called a twistor (of $H$ ) if it satisfies equations (i) and (ii) in Proposition 3.2.5 and it is invertible (in the just explained sense). 
Let now $\mathcal{F}$ be a twistor of $H$. Then we may define a new coproduct $\Delta_{\mathcal{F}}: H \longrightarrow$ $H \otimes_{A_{\mathcal{F}}} H$ of $H$ by the formula $\Delta_{\mathcal{F}}(x):=\left(\mathcal{F}^{\#}\right)^{-1}(\Delta(x) \cdot \mathcal{F})$. The key result is then the following (see [34]):

Theorem 3.2.8. Let $\left(H, A, s^{\ell}, t^{\ell}, m, \Delta, \epsilon\right)$ be a left bialgebroid. Then $\left(H, A_{\mathcal{F}}, s_{\mathcal{F}}^{\ell}, t_{\mathcal{F}}^{\ell}, m, \Delta_{\mathcal{F}}, \epsilon\right)$ is a left bialgebroid as well.

3.2.9. Left bialgebroid structures on universal enveloping algebras $V^{\ell}(L)$. Given a Lie-Rinehart algebra $L$, there is a standard left bialgebroid structure on $V^{\ell}(L)$.

Source and target maps are equal and given by $\iota_{A}: A \longrightarrow V^{\ell}(L)$. Then the $A^{e}$-module structure $\nabla^{\ell}(L)_{\triangleleft}$ is given by $a \triangleright u \triangleleft \tilde{a}:=a \tilde{a} u$. The coproduct $\Delta_{\ell}$ : $V^{\ell}(L) \longrightarrow V^{\ell}(L)_{\triangleleft} \otimes_{\triangleright} V^{\ell}(L)$ and the counit map $\epsilon: V^{\ell}(L) \longrightarrow A$ are determined by

$$
\begin{array}{ll}
\Delta_{\ell}(a)=a \otimes 1, \quad \Delta_{\ell}(X)=X \otimes 1+1 \otimes X, \quad \epsilon(a)=a, & \epsilon(X)=0 \\
& \forall a \in A, X \in L .
\end{array}
$$

Note that the anchor map $\omega$ endows $A$ with an obvious left $V^{\ell}(L)$-module structure, given by $u . a:=\omega(u)(a)$ for $u \in V^{\ell}(L), a \in A$, that coincides with the anchor of the left bialgebroid $V^{\ell}(L)$; cf. Remarks 3.2.2(b).

More in general, left $V^{\ell}(L)$-module structures on $A$ correspond to left bialgebroid structures on $V^{\ell}(L)$ over $A$ (see [22]). Finally, one can recover the anchor of $L$ from the left bialgebroid structure of $V^{\ell}(L)$ as follows: $\omega_{L}(X)(a)=\epsilon_{V} \ell_{(L)}(X a)$ for all $X \in L, a \in A$.

Remark 3.2.10. Let $(A, L)$ and $\left(A^{\prime}, L^{\prime}\right)$ be two Lie-Rinehart algebras. Endow $V^{\ell}(L)$ and $V^{\ell}\left(L^{\prime}\right)$ with their standard left bialgebroid structure. A Lie-Rinehart algebra morphism from $(A, L)$ to $\left(A^{\prime}, L^{\prime}\right)$ as it was defined in [15] - see also "morphisms of Lie pseudo-algebras" as they are defined in [13] — gives rise to a left bialgebroid morphism from $V^{\ell}(L)$ to $V^{\ell}\left(L^{\prime}\right)$.

Our next theorem is a suitable version for left bialgebroids of the well-known Cartier-Milnor-Moore theorem (for Hopf algebras). A similar result is given in [28], yet in this paper we do need (later on) that kind of result exactly as stated here below.

Theorem 3.2.11. Assume that $A$ is a unital commutative algebra over the field $k$.

(a) Let $\left(U, A, s^{\ell}, t^{\ell}, \Delta_{\ell}, \epsilon\right)$ be a left bialgebroid such that $s^{\ell}=t^{\ell}$. Set

$$
P^{\ell}(U):=\left\{u \in U \mid \Delta_{\ell}(u)=u \otimes 1+1 \otimes u\right\}
$$

(the set of "left primitive elements" of $U$ ). Then the pair $\left(A, P^{\ell}(U)\right)$ is a LieRinehart algebra.

(b) Assume in addition that $P^{\ell}(U)$ is projective as an $A-m o d u l e$, and that $P^{\ell}(U)$ and $s^{\ell}(A)$ generate $U$ as an algebra. Then $U$ is isomorphic to $V^{\ell}\left(P^{\ell}(U)\right)$ as a left bialgebroid. 
Proof. (a) On $P^{\ell}(U)$ we set the following $A$-module structure: $a \cdot D:=s^{\ell}(a) D$ for all $a \in A, D \in P^{\ell}(U)$. Moreover, if $D, D^{\prime} \in P^{\ell}(U)$ then $\left[D, D^{\prime}\right]:=D \cdot D^{\prime}-$ $D^{\prime} \cdot D \in P^{\ell}(U)$, by direct check: this defines a Lie bracket on $P^{\ell}(U)$. Finally, we define $\omega: P^{\ell}(U) \longrightarrow \operatorname{Der}(A)$ by $D \mapsto\left(b \stackrel{\omega(D)}{\longrightarrow} \epsilon\left(D s^{\ell}(b)\right)\right)$. It is proved in [22] (Proposition 4.2.1) that $\left(A, P^{\ell}(U), \omega\right)$ is a Lie-Rinehart algebra.

(b) By assumption, the natural algebra morphism from $T_{k}\left(A \oplus P^{\ell}(U)\right)$ to $U$ is surjective and it induces a surjective algebra morphism $f: V^{\ell}\left(P^{\ell}(U)\right) \rightarrow U$. As $P^{\ell}(U)$ and $s^{\ell}(A)$ generates $V^{\ell}\left(P^{\ell}(U)\right)$ as an algebra, this map is also a morphism of corings. By the same argument as in [27], Lemma 5.3.3, one shows that $f$ is also injective because $\left.f\right|_{P^{\ell}(U)}$ is injective (which is obvious).

3.3. Right bialgebroids. Just like for left bialgebroids, one can consider the notion of right bialgebroids (cf. [17] and [4]). We will need a second type of "Takeuchi product". In order to distinguish it from the previous one, we shall now denote the base $k$-algebra by $B$ instead of $A$. Hereafter, $B$ is a (unital, associative) $k$-algebra, and we use notations as in $\S 3.1$. Let $H$ be a $B^{e}$-ring given by a $k$-algebra morphism $\eta^{r}: B^{e} \longrightarrow H$, a source map $s^{r}:=\eta^{r}(-\otimes 1)$ and a target map $t^{r}:=\eta^{r}(1 \otimes-)$. We consider now the right $B^{e}$-module structure on $H$ given by $h \cdot(b \otimes \tilde{b}):=h \cdot \eta^{r}(b \otimes \tilde{b})$, for $b, \tilde{b} \in B, h \in H$. Then the tensor product of $H$ with itself (as a $B$-bimodule, i.e. a $B^{e}$-module) is defined as

$$
H \triangleleft_{B} \otimes_{\triangleright} H:=H \otimes_{k} H /\left\{(u \triangleleft b) \otimes u^{\prime}-u \otimes\left(b \triangleright u^{\prime}\right)\right\}_{b \in B, u, u^{\prime} \in H}
$$

Now we define the right Takeuchi product (of the $B^{e}$-ring $H$ with itself) $H_{\triangleleft}{ }_{B} H \subseteq$ $H \triangleleft_{B} \otimes_{\triangleright} H$

$$
H \underset{B}{\times} H:=\left\{\sum_{i} u_{i} \otimes u_{i}^{\prime} \in H_{\triangleleft} \stackrel{B}{\otimes} H \mid \sum_{i}\left(a \triangleright u_{i}\right) \otimes u_{i}^{\prime}=\sum_{i} u_{i} \otimes\left(u_{i}^{\prime} \triangleleft a\right)\right\}
$$

Definition 3.3.1. A right $B$-bialgebroid is a $k$-module $H$ that carries simultaneously a structure of a $B^{e}$-ring $\left(H, s^{r}, t^{r}\right)$ and of a $B$-coring $\left(H, \Delta_{r}, \partial\right)$ subject to the following compatibility relations:

(i) The $B^{e}$-module structure on the $B$-coring $\left(H, s^{r}, t^{r}\right)$ is that of $H_{\triangleleft}$, namely (for all $b, \tilde{b} \in B, h \in H,) b \bullet h \triangleleft \tilde{b}:=h s^{r}(\tilde{b}) t^{r}(b)=h \eta(\tilde{b} \otimes b)$.

(ii) The coproduct map $\Delta_{r}$ is a unital $k$-algebra morphism taking values in $H \stackrel{B}{\times}$ \ $H$. 
(iii) The (right) counit map $\partial$ has the following property: for all $b, \tilde{b} \in B$, $u, u^{\prime} \in H$, one has

$$
\begin{gathered}
\partial\left(u s^{r}(\tilde{b}) t^{r}(b)\right)=b \partial(u) \tilde{b}, \\
\partial\left(u u^{\prime}\right)=\partial\left(s^{r}(\partial(u)) u^{\prime}\right)=\partial\left(t^{r}(\partial(u)) u^{\prime}\right), \\
\partial(1)=1
\end{gathered}
$$

Remarks 3.3.2. (a) The definition of a right bialgebroid is obtained from the definition of a left bialgebroid by exchanging the role of black triangles and white triangles $(\triangleright, \triangleleft)$. Consequently, the properties of a right bialgebroid are obtained from those of a left bialgebroid - see Remarks 3.2.2 - by exchanging the roles of black triangles and white triangles.

(b) (cf. [22]) The "opposite" of a left bialgebroid $U=\left(U, A, s^{\ell}, t^{\ell}, \Delta_{\ell}, \epsilon\right)$ is defined as $U^{o p}:=\left(U^{o p}, A, t^{\ell}, s^{\ell}, \Delta_{\ell}, \epsilon\right)$ : this can be shown to be a right bialgebroid. The "coopposite" is given by $U_{\text {coop }}:=\left(U, A^{o p}, t^{\ell}, s^{\ell}, \Delta_{\ell}^{\text {coop }}, \epsilon\right)$ with $\Delta_{\ell}^{\text {coop }}: U \longrightarrow$ $\triangleright U \otimes_{A^{o p}} U_{\triangleleft}, u \mapsto u_{(2)} \otimes u_{(1)}$; this is still a left bialgebroid. As a consequence, $U_{c o o p}^{o p}$ is a right bialgebroid.

The definition of a right bialgebroid morphism is analogous to that of a left bialgebroid morphism. We denote by (RBialg) the category of right bialgebroids, whose objects are right bialgebroids and morphisms are defined mimicking Definition 3.2.3. Inside it, (RBialg) ${ }_{A}$ is the subcategory whose objects are all the right bialgebroids over $A$ and whose morphisms are all those in (RBialg) of the form (id, $F$ ).

3.3.3. Right bialgebroid structures on universal enveloping algebras $V^{r}(L)$. Given a Lie-Rinehart algebra $L$, now considered as a right one, its right universal enveloping algebra $V^{r}(L)$ bears a natural structure of right bialgebroid over $A$ obtained by pulling-back the left bialgebroid structure of $V^{\ell}(L)$ via the isomorphism $V^{r}(L) \cong V^{\ell}(L)^{o p}$. More explicitly, the $A^{e}$-module structure $\neg V^{r}(L)_{\triangleleft}$ is given by $a \triangleright u \triangleleft \tilde{a}:=u a \tilde{a}$; the coproduct $\Delta_{r}: V^{r}(L) \longrightarrow V^{r}(L) \bigotimes_{A} \otimes^{r}(L)$ and the counit $\partial: V^{r}(L) \longrightarrow A$ are determined by

$$
\begin{array}{ll}
\Delta_{r}(a)=a \otimes 1, \quad \Delta_{r}(X)=X \otimes 1+1 \otimes X, \quad \partial(a)=a, & \partial(X)=0 \\
& \forall a \in A, X \in L .
\end{array}
$$

Finally, one can recover the anchor map of $L$ from the right bialgebroid structure of $V^{r}(L)$ as $\omega_{L}(X)(a)=-\partial_{V} r_{(L)}(a X)$ for all $X \in L, a \in A$. 
We also have an analogue for right bialgebroids of Theorem 3.2.11 (with similar proof):

Theorem 3.3.4. Assume that $A$ is a unital commutative algebra over the field $k$. (a) Let $\left(W, A, s^{r}, t^{r}, \Delta_{r}, \partial\right)$ be a right bialgebroid such that $s^{r}=t^{r}$. Set

$$
P^{r}(W):=\left\{w \in W \mid \Delta_{r}(w)=w \otimes 1+1 \otimes w\right\}
$$

(the set of "right primitive elements" of $W$ ). Then the pair $\left(A, P^{r}(W)\right)$ is a right Lie-Rinehart algebra for the following right action and anchor map

$$
w \cdot a:=w s^{r}(a), \quad \omega(D)(a):=-\partial\left(s^{r}(a) D\right), \quad \forall w \in W, \forall D \in P^{r}(W), \forall a \in A
$$

(b) Assume in addition that $P^{r}(W)$ is projective as an A-module, and that $P^{r}(W)$ and $s^{r}(A)$ generate $W$ as an algebra. Then $W$ is isomorphic to $V^{r}\left(P^{r}(W)\right)$ as a right bialgebroid.

Remark 3.3.5. The previous result improves a bit as follows.

Let $\left(W, A, s^{r}, t^{r}, \Delta_{r}, \partial\right)$ be a right bialgebroid for which $A$ is commutative and $s^{r}=t^{r}$. Let $Q \subseteq P^{r}(U)$ be a right Lie-Rinehart subalgebra of $P^{r}(W)$ such that (i) $Q$ is a projective $A$-module and (ii) $Q$ and $A$ generate $W$ as an algebra. Then $W$ is isomorphic to $V^{r}(Q)$ as a right bialgebroid, and $Q=P^{r}(W)$.

An entirely similar remark also applies to Theorem 3.2.11.

3.4. Duals of bialgebroids. We shall now consider left and right dual of (left and right) bialgebroids, and investigate their main properties. We begin with dual of left bialgebroids, then we pass on to dual of right ones.

Definition 3.4.1. (a) Let $U$ be a left $A$-bialgebroid, with structure maps as before.

The left dual and the right dual of $U$ respectively are the spaces

$$
\begin{aligned}
U_{*}: & =\left\{\phi: U \longrightarrow A \mid \phi\left(u^{\prime}+u^{\prime \prime}\right)=\phi\left(u^{\prime}\right)+\phi\left(u^{\prime \prime}\right), \phi\left(s^{\ell}(a) u\right)=a \phi(u)\right\} \\
& =\operatorname{Hom}_{A}\left({ }_{\triangleright} U,{ }_{A} A\right) \\
U^{*}: & =\left\{\phi: U \longrightarrow A \mid \phi\left(u^{\prime}+u^{\prime \prime}\right)=\phi\left(u^{\prime}\right)+\phi\left(u^{\prime \prime}\right), \phi\left(t^{\ell}(a) u\right)=\phi(u) a\right\} \\
& =\operatorname{Hom}_{A}\left(U_{\triangleleft}, A_{A}\right)
\end{aligned}
$$

(b) Let $W$ be a right $A$-bialgebroid, with structure maps as before. The left dual and the right dual of $W$ respectively are the spaces

$$
\begin{aligned}
{ }^{*} W & :=\left\{\psi: W \longrightarrow A \mid \psi\left(w^{\prime}+w^{\prime \prime}\right)=\psi\left(w^{\prime}\right)+\psi\left(w^{\prime \prime}\right), \psi\left(w t^{r}(a)\right)=a \psi(w)\right\} \\
& =\operatorname{Hom}_{A}\left(\downarrow W,{ }_{A} A\right) \\
{ }^{*} W: & =\left\{\psi: W \longrightarrow A \mid \psi\left(w^{\prime}+w^{\prime \prime}\right)=\psi\left(w^{\prime}\right)+\psi\left(w^{\prime \prime}\right), \psi\left(w s^{r}(a)\right)=\psi(w) a\right\} \\
& =\operatorname{Hom}_{A}\left(W_{\triangleleft}, A_{A}\right)
\end{aligned}
$$


3.4.2. Bialgebroid structures on dual spaces. Let $U$ be a left $A$-bialgebroid as above. We shall now introduce on its dual spaces $U_{*}$ and $U^{*}$ a structure of right $A$-bialgebroid; most of the structure is well-defined in general, but for the coproduct we need an additional assumption, namely $U$ as an $A$-module (on the left, or the right, see below) has to be projective.

Product structure. First we recall (see [17], and also [22]) that $U_{*}$ and $U^{*}$ can be equipped with a product, for which the counit map $\epsilon$ is a two-sided unit. For $\phi, \phi^{\prime} \in U_{*}, \psi, \psi^{\prime} \in U^{*}, u \in U$, set

$$
\begin{aligned}
\left(\phi \phi^{\prime}\right)(u) \equiv m_{U_{*}}\left(\phi \otimes \phi^{\prime}\right)(u): & =\phi^{\prime}\left(m_{U^{o p}}\left(i d \otimes\left(t^{\ell} \circ \phi\right)\right)\left(\Delta_{\ell}(u)\right)\right) \\
& =\phi^{\prime}\left(t^{\ell}\left(\phi\left(u_{(2)}\right)\right) \cdot u_{(1)}\right) \\
\left(\psi \psi^{\prime}\right)(u) \equiv m_{U^{*}}\left(\psi \otimes \psi^{\prime}\right)(u) & :=\psi^{\prime}\left(m_{U}\left(\left(s^{\ell} \circ \psi\right) \otimes i d\right)\left(\Delta_{\ell}(u)\right)\right) \\
& =\psi^{\prime}\left(s^{\ell}\left(\psi\left(u_{(1)}\right)\right) \cdot u_{(2)}\right)
\end{aligned}
$$

$A$-module structures. For the left dual space $U_{*}$, the left dual source map $s_{*}^{r}$ : $A \longrightarrow U_{*}$ and the right dual target map $t_{*}^{r}: A \longrightarrow U_{*}$ are defined as follows:

$$
\left(s_{*}^{r}(a)\right)(u):=\epsilon\left(t^{\ell}(a) u\right)=\epsilon(u) a, \quad\left(t_{*}^{r}(a)\right)(u):=\epsilon\left(u t^{\ell}(a)\right) \quad \forall a \in A, u \in U .
$$

Then one has, in the usual way, two left and two right actions of $A$ on $U_{*}$, given by

$$
\begin{aligned}
& (a \triangleright \phi)(u):=\left(s_{*}^{r}(a) \phi\right)(u)=\phi\left(t^{\ell}(a) u\right), \\
& (\phi \triangleleft a)(u):=\left(t_{*}^{r}(a) \phi\right)(u)=\phi\left(u t^{\ell}(a)\right) \\
& (a \triangleright \phi)(u):=\left(\phi t_{*}^{r}(a)\right)(u)=\phi\left(u s^{\ell}(a)\right), \\
& (\phi \triangleleft a)(u):=\left(\phi s_{*}^{r}(a)\right)(u)=\phi(u) a
\end{aligned}
$$

Similarly, for the right dual $U^{*}$ the source $s_{r}^{*}: A \longrightarrow U^{*}$ and the target $t_{r}^{*}: A \longrightarrow U^{*}$ are

$$
\left(s_{r}^{*}(a)\right)(u):=\epsilon\left(u s^{\ell}(a)\right), \quad\left(t_{r}^{*}(a)\right)(u):=\epsilon\left(s^{\ell}(a) u\right)=a \epsilon(u) \quad \forall a \in A, u \in U .
$$

Then one has, like before, two left and two right $A$-actions on $U^{*}$, given by

$$
\begin{aligned}
(a \triangleright \psi)(u) & :=\left(s_{r}^{*}(a) \psi\right)(u)=\psi\left(u s^{\ell}(a)\right), \\
(\psi \triangleleft a)(u) & :=\left(t_{r}^{*}(a) \psi\right)(u)=\psi\left(s^{\ell}(a) u\right) \\
(a \triangleright \psi)(u) & :=\left(\psi t_{r}^{*}(a)\right)(u)=a \psi(u), \\
(\psi \triangleleft a)(u) & :=\left(\psi s_{r}^{*}(a)\right)(u)=\psi\left(u t^{\ell}(a)\right)
\end{aligned}
$$


Coproduct structure. Now assume that ${ }_{\triangleright} U$ as an A-module be projective. Then we now endow the left dual $U_{*}$ with a coproduct $\Delta_{r}$ which, eventually, makes it into a right bialgebroid.

Consider the injective map $\chi: U_{* \triangleleft} \otimes{ }_{\triangleright} U_{*} \longrightarrow \operatorname{Hom}_{(A,-)}\left(\triangleright\left(U_{\triangleleft} \otimes \triangleright U\right),{ }_{A} A\right)$ given by

$$
\phi \otimes \phi^{\prime} \mapsto \chi\left(\phi \otimes \phi^{\prime}\right)\left(u \otimes u^{\prime} \mapsto \chi\left(\phi \otimes \phi^{\prime}\right)\left(u \otimes u^{\prime}\right):=\phi^{\prime}\left(u s^{\ell}\left(\phi\left(u^{\prime}\right)\right)\right)\right)
$$

Now, if $U$ is finite projective (as an $A$-module) then the previous map is even an isomorphism. If instead $U$ is projective but not finite, one can endow $U_{* \triangleleft} \otimes U_{*}$ with a suitable topology (typically, the "weak" one), and denote by $U_{*} \widetilde{\otimes} \nabla_{*}$ the corresponding completion: then the above map extends - by continuity, using the notion of basis for a projective module (cf. [2]) - to an isomorphism from $U_{* \triangleleft} \widetilde{\otimes} U_{*}$ to $\operatorname{Hom}_{(A,-)}\left(\triangleright\left(U_{\triangleleft} \otimes \triangleright U\right),{ }_{A} A\right)$. This allows us to define a coproduct $\Delta_{r}$ on $U_{*}$ as the transpose of the multiplication on $U$, namely

$$
\begin{aligned}
\Delta_{r}: U_{*} \longrightarrow \operatorname{Hom}_{(A,-)}\left(\triangleright\left(U_{\triangleleft} \otimes \triangleright U\right),{ }_{A} A\right) \underset{\chi^{-1}}{\cong} U_{* \triangleleft} \widetilde{\otimes_{\triangleright}} U_{*}, \\
\phi \mapsto \Delta_{r}(\phi)\left(u \otimes u^{\prime} \mapsto \phi\left(u u^{\prime}\right)\right)
\end{aligned}
$$

This coproduct makes $U_{*}$ into a (topological) $A$-coring, with counit $\eta_{*}: U_{*} \longrightarrow A$, $\eta_{*}(\phi):=\phi(1)$. Similarly, if $U_{\triangleleft}$ as an $A$-module is projective, then for its right dual $U^{*}$ a coproduct is defined as follows. Consider the injective map $\vartheta: U_{\triangleleft}^{*} \otimes U^{*} \longrightarrow$ $\operatorname{Hom}_{(-, A)}\left(\left(U_{\triangleleft} \otimes \triangleleft U\right)_{\triangleleft}, A_{A}\right)$ given by

$$
\psi \otimes \psi^{\prime} \mapsto \vartheta\left(\psi \otimes \psi^{\prime}\right)\left(u \otimes u^{\prime} \mapsto \vartheta\left(\psi \otimes \psi^{\prime}\right)\left(u \otimes u^{\prime}\right):=\psi\left(u^{\prime} t^{\ell}\left(\psi^{\prime}(u)\right)\right)\right)
$$

Again, if $U$ is finite projective (as an $A$-module) then this map is an isomorphism. If instead $U$ is projective but not finite, one can endow $U^{*} \triangleleft \otimes U^{*}$ with a suitable topology (like the weak one), and denote by $U^{*} \widetilde{\otimes}_{\nabla} U^{*}$ the corresponding completion: then the above map extends — by continuity — to an isomorphism $\widetilde{\vartheta}$ from $U^{*} \widetilde{\otimes}_{\triangleleft} U^{*}$ to $\operatorname{Hom}_{(-, A)}\left(\left(U_{\triangleleft} \otimes \triangleleft U\right)_{\triangleleft}, A_{A}\right)$. Thus we can define a coproduct $\Delta_{r}$ on $U^{*}$ as the transpose of the opposite multiplication on $U$, namely

$$
\begin{aligned}
& \Delta_{r}: U^{*} \longrightarrow \operatorname{Hom}_{(-, A)}\left(\left(U_{\triangleleft} \otimes \triangleleft U\right)_{\triangleleft}, A_{A}\right) \underset{\widetilde{\vartheta}-1}{\cong} U^{*} \widetilde{\otimes}_{\triangleright} U^{*}, \\
& \psi \mapsto \Delta_{r}(\psi)\left(u \otimes u^{\prime} \mapsto \psi\left(u^{\prime} u\right)\right)
\end{aligned}
$$

This makes $U^{*}$ into a (topological) $A$-coring, with counit $\partial_{*}: U^{*} \longrightarrow A$ given by $\partial_{*}(\psi):=\psi(1)$.

Conclusion. If $U$ is any left bialgebroid over $A$, projective as an $A$-module, then $U_{*}$ and $U^{*}$ with the structures introduced above are both (topological) right bialgebroids over $A$. 
Similarly, we consider the case of a right $A$-bialgebroid $W$, and we introduce canonical structures of (topological) left $A$-bialgebroids on its left and right dual spaces $* W$ and ${ }^{*} W$ : indeed, everything is strictly similar to what occurs in the previous case for $U$, so we skip details.

Notation. In the following, if $v$ is an element of some (left or right) $A$-module, and $\phi$ is an element of the (left or right) dual of that module, we shall write $\langle\phi, v\rangle:=\phi(v)$ or $\langle v, \phi\rangle:=\phi(v)$.

Remark 3.4.3. If $U$ is a left bialgebroid which is projective of finite type as an $A$ module, then it is isomorphic to ${ }^{*}\left(U_{*}\right)$ and to $*\left(U^{*}\right)-$ as a left bialgebroid. This follows from the equalities

$$
\left\langle u, \phi s_{*}^{r}(a)\right\rangle=\langle u, \phi| a, \quad\left\langle u, \psi t_{r}^{*}(a)\right\rangle=a\langle u, \psi\rangle \quad \forall a \in A, u \in U, \phi \in U_{*}, \psi \in U^{*}
$$

We introduce now the natural vocabulary of "pairings", which we shall use in computations.

Definition 3.4.4. (a) Let $\left(U, s_{\ell}, t_{\ell}\right)$ and $\left(W, s^{r}, t^{r}\right)$ be two $A^{e}$-modules. An $A^{e}$-left pairing is a $k$-bilinear map $\langle\rangle:, U \times W \longrightarrow A$ such that, for any $u \in U, w \in W$ and $a \in A$, one has

$$
\begin{gathered}
\langle u, a \triangleright w\rangle=\left\langle u, s^{r}(a) w\right\rangle=\left\langle t_{\ell}(a) u, w\right\rangle=\langle u \triangleleft a, w\rangle \\
\langle u, w \triangleleft a\rangle=\left\langle u, t^{r}(a) w\right\rangle=\left\langle u t_{\ell}(a), w\right\rangle=\langle a \triangleright u, w\rangle \\
\langle u, a \triangleright w\rangle=\left\langle u, w t^{r}(a)\right\rangle=\left\langle u s_{\ell}(a), w\right\rangle=\langle u \triangleleft a, w\rangle \\
\langle u, w \triangleleft a\rangle=\left\langle u, w s^{r}(a)\right\rangle=\langle u, w\rangle a \\
\langle a \triangleright u, w\rangle=\left\langle s^{\ell}(a) u, w\right\rangle=a\langle u, w\rangle
\end{gathered}
$$

Then there exist natural morphisms of $A^{e}$-modules $W \longrightarrow U_{*}$ and $U \longrightarrow{ }^{*} W$. The pairing is non degenerate if the left and right kernels of this pairing are trivial, that is to say

$$
\langle u, w\rangle=0, \forall w \in W \Longrightarrow u=0, \quad\langle u, w\rangle=0, \forall u \in U \Longrightarrow w=0
$$

In other words, the pairing is non degenerate if and only if the above maps $W \longrightarrow U_{*}$ and $U \longrightarrow{ }^{*} W$ (which are morphisms of $A^{e}$-modules) are injective. 
(b) Let $\left(U, s^{\ell}, t^{\ell}\right)$ and $\left(W, s_{r}, t_{r}\right)$ be two $A^{e}$-modules. An $A^{e}$-right pairing is a $k$-bilinear map $\langle\rangle:, U \times W \longrightarrow A$ such that, for any $u \in U, w \in W$ and $a \in A$, one has

$$
\begin{gathered}
\langle u, w \triangleleft a\rangle=\left\langle u, t_{r}(a) w\right\rangle=\left\langle s^{\ell}(a) u, w\right\rangle=\langle a \triangleright u, w\rangle \\
\langle u, a \triangleright w\rangle=\left\langle u, s_{r}(a) w\right\rangle=\left\langle u s^{\ell}(a), w\right\rangle=\langle u \triangleleft a, w\rangle \\
\langle u, w \triangleleft a\rangle=\left\langle u, w s_{r}(a)\right\rangle=\left\langle u t^{\ell}(a), w\right\rangle=\langle a \triangleright u, w\rangle \\
\langle u, a \triangleright w\rangle=\left\langle u, w t_{r}(a)\right\rangle=a\langle u, w\rangle \\
\langle u \triangleleft a, w\rangle=\left\langle t^{\ell}(a) u, w\right\rangle=\langle u, w\rangle a
\end{gathered}
$$

Then there exist natural morphisms of $A^{e}$-modules $W \longrightarrow U^{*}$ and $U \longrightarrow{ }_{*} W$. The pairing is non degenerate if the left and right kernels of this pairing are trivial, that is to say

$$
\langle u, w\rangle=0, \forall w \in W \Longrightarrow u=0, \quad\langle u, w\rangle=0, \forall u \in U \Longrightarrow w=0
$$

In other words, the pairing is non degenerate if and only if the above maps $W \longrightarrow U^{*}$ and $U \longrightarrow{ }_{*} W$ (which are morphisms of $A^{e}$-modules) are injective.

Definition 3.4.5. (a) Let $\left(U, s_{\ell}, t_{\ell}, \Delta, \epsilon\right)$ be a left $A$-bialgebroid and $\left(W, s^{r}, t^{r}, \Delta, \eta\right)$ be a right $A$-bialgebroid. A bialgebroid left pairing is a non degenerate $A^{e}$-left pairing $\langle\rangle:, U \times W \longrightarrow A$ such that

$$
\begin{array}{ll}
\left\langle u u^{\prime}, w\right\rangle=\left\langle u, w_{(2)} t^{r}\left(\left\langle u^{\prime}, w_{(1)}\right)\right)\right\rangle=\left\langle u s_{\ell}\left(\left\langle u^{\prime}, w_{(1)}\right)\right), w_{(2)}\right\rangle, & \langle 1, w\rangle=\eta(w) \\
\left\langle u, w w^{\prime}\right\rangle=\left\langle t^{\ell}\left(\left\langle u_{(2)}, w\right\rangle\right) u_{(1)}, w^{\prime}\right\rangle=\left\langle u_{(1)}, s_{r}\left(\left\langle u_{(2)}, w\right\rangle\right) w^{\prime}\right\rangle, & \langle u, 1\rangle=\epsilon(u)
\end{array}
$$

for any $u, u^{\prime} \in U$ and any $w, w^{\prime} \in W$. In other words, the natural maps $W \longrightarrow U_{*}$ and $U \longrightarrow{ }^{*} W$ are (injective) morphisms of right and left bialgebroids respectively.

(b) Let $\left(U, s^{\ell}, t^{\ell}, \Delta, \epsilon\right)$ be a left $A$-bialgebroid and $\left(W, s_{r}, t_{r}, \Delta, \eta\right)$ be a right $A-$ bialgebroid. A bialgebroid right pairing is a non degenerate $A^{e}$-right pairing $\langle$,$\rangle :$ $U \times W \longrightarrow A$ such that

$$
\begin{array}{ll}
\left\langle u u^{\prime}, w\right\rangle=\left\langle u t_{\ell}\left(\left\langle u^{\prime}, w_{(2)}\right)\right), w_{(1)}\right\rangle=\left\langle u, w_{(1)} s^{r}\left(\left\langle u^{\prime}, w_{(2)}\right)\right)\right\rangle, & \langle 1, w\rangle=\eta(w) \\
\left\langle u, w w^{\prime}\right\rangle=\left\langle s^{\ell}\left(\left\langle u_{(1)}, w\right\rangle\right) u_{(2)}, w^{\prime}\right\rangle=\left\langle u_{(2)}, t_{r}\left(\left\langle u_{(1)}, w\right\rangle\right) w^{\prime}\right\rangle, & \langle u, 1\rangle=\epsilon(u)
\end{array}
$$

for any $u, u^{\prime} \in U$ and any $w, w^{\prime} \in W$. In other words, the natural maps $W \longrightarrow U^{*}$ and $U \longrightarrow{ }_{*} W$ are (injective) morphisms of right and left bialgebroids respectively. 
Remarks 3.4.6. (a) If $U$ is a left bialgebroid, then the couple $\left(U, U^{*}\right)$ bears a bialgebroid right pairing, whereas $\left(U, U_{*}\right)$ bears a bialgebroid left pairing.

(b) Let $U$ be a left bialgebroid. Then the left bialgebroids $\left(U^{*}\right)_{\text {coop }}^{o p}$ and ${ }_{*}\left(U_{\text {coop }}^{o p}\right)$ are isomorphic: indeed, the right $A^{e}$-pairings between $U$ and $U^{*}$ and between * $\left(U_{\text {coop }}^{o p}\right)$ and $U_{\text {coop }}^{o p}$ give rise to the same formulas. Similarly, the left bialgebroids $\left(U_{*}\right)_{\text {coop }}^{o p}$ and $*\left(U_{\text {coop }}^{o p}\right)$ are isomorphic.

\subsection{The jet space(s) of a Lie-Rinehart algebra.}

3.5.1. Bialgebroids of jets: the right version. Let $(L, A)$ be a Lie-Rinehart algebra, projective as an $A$-module. Consider its enveloping algebra $V^{\ell}(L)$ endowed with its standard left bialgebroid structure and define the right jet space of the Lie-Rinehart algebra $L$ as

$$
J^{r}(L):=V^{\ell}(L)^{*}=\operatorname{Hom}_{(-, A)}\left(V^{\ell}(L)_{\triangleleft}, A_{A}\right)
$$

As in \$3.4.2, a multiplication in $J^{r}(L)$ can be given by $\left(\phi \phi^{\prime}\right)(u)=\phi\left(u_{(1)}\right) \phi^{\prime}\left(u_{(2)}\right)$ for $\phi, \phi^{\prime} \in J^{r}(L), u \in V^{\ell}(L)$. In particular, this multiplication is commutative, and the counit map of $V^{\ell}(L)$ is the unit element of $J^{r}(L)$. Also, the map $\partial=\partial_{J^{r}(L)}$ : $J^{r}(L) \longrightarrow A, \phi \mapsto \partial(\phi):=\phi\left(1_{V^{\ell}(L)}\right)$, will play the role of counit map of $J^{r}(L)$; hereafter, we write $\mathfrak{J}_{J^{r}(L)}:=\operatorname{Ker}\left(\partial_{J^{r}}{ }_{(L)}\right)$. Moreover, we have a structure of $A^{e}$-ring on $J^{r}(L)$, whose source and target maps are given - for all $a \in A, u \in V^{\ell}(L)$ by the formulas $\left(s^{r}(a)\right)(u):=\epsilon\left(u s^{\ell}(a)\right),\left(t^{r}(a)\right)(u):=\epsilon\left(s^{\ell}(a) u\right)=a \epsilon(u)$. Note that $J^{r}(L)$ is complete for the $\mathfrak{J}_{J^{r}(L)}$-adic topology.

To define a coproduct on $J^{r}(L):=V^{\ell}(L)^{*}$ we adapt the construction in $\$ 3.4 .2$ (cf. also [22], [24], [6]). Consider the injective map $\vartheta: J^{r}(L) \triangleleft \otimes_{\triangleleft} J^{r}(L)=$ $V^{\ell}(L)_{\triangleleft}^{*} \otimes V^{\ell}(L)^{*} \longrightarrow\left(V^{\ell}(L)_{\triangleleft} \otimes V^{\ell}(L)\right)^{*}$ given (as in \$3.4.2) by $\psi \otimes \psi^{\prime} \mapsto$ $\vartheta\left(\psi \otimes \psi^{\prime}\right)\left(u \otimes u^{\prime} \mapsto \vartheta\left(\psi \otimes \psi^{\prime}\right)\left(u \otimes u^{\prime}\right):=\psi\left(u^{\prime} t^{\ell}\left(\psi^{\prime}(u)\right)\right)\right)$.

Consider in $J^{r}(L) \otimes J^{r}(L)$ the $\mathfrak{J}_{\otimes}$-adic filtration, with $\mathfrak{J}_{\otimes}:=\mathfrak{J}_{J} r_{(L)} \otimes$ $J^{r}(L)+J^{r}(L) \otimes \mathfrak{J}_{J} r_{(L)}=\operatorname{Ker}\left(\partial_{J^{r}(L)} \otimes \partial_{J^{r}(L)}\right)$, and the corresponding topology defined by it in $J^{r}(L), \otimes J^{r}(L)$; then denote by $J^{r}(L), \widetilde{\otimes} J^{r}(L)$ the $\mathfrak{J}_{\otimes}$-adic completion of $J^{r}(L) \triangleleft \otimes_{\triangleleft} J^{r}(L)$. The completion of $\vartheta, \widetilde{\vartheta}: J^{r}(L) \triangleleft \widetilde{\otimes} J^{r}(L) \longrightarrow$ $\left(V^{\ell}(L)_{\triangleleft} \otimes V^{\ell}(L)\right)^{*}$, is an isomorphism. Therefore, we can complete the procedure explained in $\$ 3.4 .2$ and define a coproduct $\Delta: J^{r}(L) \longrightarrow J^{r}(L) \widetilde{\otimes}_{\nabla} J^{r}(L)$ on $J^{r}(L):=V^{\ell}(L)^{*}$ as $\Delta:=\widetilde{\vartheta}^{-1} \circ \nabla$ where $\nabla: J^{r}(L):=V^{\ell}(L)^{*} \longrightarrow$ $\left(V^{\ell}(L)_{\triangleleft} \otimes V^{\ell}(L)\right)^{*}$ is given by $\nabla: \psi \mapsto \nabla(\psi)\left(u \otimes u^{\prime} \mapsto \psi\left(u^{\prime} u\right)\right)$ for all $\psi \in J^{r}(L), u, u^{\prime} \in V^{\ell}(L)$. As an outcome, we have

$$
\Delta(\psi)=\psi_{(1)} \otimes \psi_{(2)} \in J^{r}(L) \widetilde{\otimes}_{\downarrow} J^{r}(L) \quad \text { with } \quad \psi_{(1)}\left(u t^{\ell}\left(\psi_{(2)}\left(u^{\prime}\right)\right)\right)=\psi\left(u u^{\prime}\right)
$$

This $\Delta$ makes $J^{r}(L)$ into a (topological) $A$-coring, with counit map $\partial=\partial_{J^{r}(L)}$ : $J^{r}(L) \longrightarrow A$ given as above by $\phi \mapsto \partial(\phi):=\phi\left(1_{V} \ell_{(L)}\right)$. All in all, this makes $J^{r}(L)$ into a right bialgebroid over $A$. 
Remarks 3.5.2. We have to mention some extra features of the right bialgebroid $J^{r}(L):=V^{\ell}(L)^{*}$, namely the following:

(a) as $J^{r}(L)$ is commutative, it is equal to $J^{r}(L)^{o p}$ hence it is also a left bialgebroid;

(b) it is known that $J^{r}(L)$ is a Hopf algebroid (see [22], [6], [29]): in particular, there exists a standard right bialgebroid isomorphism - called the "antipode" — from $J^{r}(L)$ to $J^{r}(L)_{\text {coop }}$.

3.5.3. Bialgebroids of jets: the left version. Let again $L$ be a Lie-Rinehart algebra over $A$, again projective as an $A$-module. Considering now $L$ as a right $A$-module, we look at its right enveloping algebra $V^{r}(L)$, endowed with its natural structure of right bialgebroid (cf. §3.3.3).

We define the left jet space of the Lie-Rinehart algebra $L$ as the left dual space

$$
J^{\ell}(L):={ }_{*} V^{r}(L)=\operatorname{Hom}_{(-, A)}\left(V^{r}(L)_{\triangleleft}, A_{A}\right)
$$

Again from $\$ 3.4 .2$ we have a multiplication in $J^{\ell}(L)$ given (for $\psi, \psi^{\prime} \in J^{\ell}(L)$, $\left.u \in V^{r}(L)\right)$ by $\left(\psi \psi^{\prime}\right)(u)=\psi\left(u_{(1)}\right) \psi^{\prime}\left(u_{(2)}\right)$; in particular this multiplication is commutative in $J^{\ell}(L)$, and the unit element of $J^{\ell}(L)$ is the counit map of $V^{r}(L)$. Moreover, the map $\epsilon=\epsilon_{J^{\ell}(L)}: J^{\ell}(L) \longrightarrow A, \psi \mapsto \epsilon(\psi):=\psi\left(1_{V^{r}(L)}\right)$, works as counit map of $J^{\ell}(L)$; in the sequel we write $\mathfrak{J}_{J} \ell_{(L)}:=\operatorname{Ker}\left(\epsilon_{J} \ell_{(L)}\right)$.

Still from $\$ 3.4 .2$ we get a structure of $A^{e}$-ring on $J^{\ell}(L)$, with source and target maps given by $\left(s^{\ell}(a)\right)(u):=\partial(a u),\left(t^{\ell}(a)\right)(u):=\partial(u) a,-$ for all $a \in A, u \in$ $V^{r}(L)$.

Finally, we can also endow $J^{\ell}(L)$ with a suitable (topological) coproduct, just adapting the recipe given in \$3.4.2. Eventually, all this makes $J^{\ell}(L)$ into a (topological) left bialgebroid.

Remark 3.5.4. As $J^{\ell}(L)=J^{r}\left(L^{o p}\right)_{\text {coop }}^{o p}$, it follows from Remarks 3.5.2 (b) that our $J^{\ell}(L):={ }_{*} V^{r}(L)$ is also a Hopf algebroid: in particular, there exists a standard right bialgebroid isomorphism — the "antipode" of $J^{\ell}(L)$ - from $J^{\ell}(L)$ to $J^{\ell}(L)_{\text {coop }}$.

3.5.5. Further jet spaces, and comparison. Besides the jet spaces $J^{r}(L)$ and $J^{\ell}(L)$, further possibilities exist. All in all we can consider four different types of "jet bialgebroids", namely

$V^{\ell}(L)^{*}=: J^{r}(L), \quad * V^{r}(L)=: J^{\ell}(L), \quad V^{\ell}(L)_{*}=:{ }^{r} J(L), \quad{ }^{*} V^{r}(L)=:{ }^{\ell} J(L)$

One can also establish some relevant links among all these bialgebroids of jets: for instance, we have already noticed that that $J^{\ell}(L) \cong J^{r}\left(L^{o p}\right)_{\text {coop }}^{o p} \cong J^{r}\left(L^{o p}\right)_{\text {coop }}$ (cf. also Remark 3.4.6). We also saw above that $V^{\ell}(L)_{*}=\left(V^{\ell}(L)^{*}\right)_{\text {coop }}=$ $J^{r}(L)_{\text {coop }} \cong J^{r}(L)$ (cf. Remarks 3.5.2) and ${ }^{*} V^{r}(L)=\left({ }_{*} V^{r}(L)\right)_{\text {coop }}=$ $J^{\ell}(L)_{\text {coop }} \cong J^{\ell}(L)$ (cf. Remark 3.5.4). Thus, in the end, jet bialgebroids of type $J^{r}(L)$ or $J^{\ell}(L)$ are enough to consider all possible situations, for every possible $L$. 

$J^{\ell}(L)$ :

We introduce now suitable "topological duals" for jet bialgebroids $J^{r}(L)$ and

Definition 3.5.6. Let $K=J^{r}(L)$ be a right jet bialgebroid, for some Lie-Rinehart algebra $L$. Set $I:=\left\{\lambda \in J^{r}(L) \mid\langle 1, \lambda\rangle=0\right\}=\operatorname{Ker}\left(\partial_{J^{r}(L)}\right)-$ which is a (twosided) ideal in $J^{r}(L)$, as one easily sees. Then we introduce the following subsets of ${ }^{*} K$ and ${ }_{*} K$ :

$$
{ }^{\star} K:=\left\{u \in{ }^{*} K \mid u\left(I^{n}\right)=0 \forall n \gg 0\right\}, \quad{ }_{\star} K:=\left\{u \in{ }_{*} K \mid u\left(I^{n}\right)=0 \forall n \gg 0\right\}
$$

Similarly, if $K:=J^{\ell}(L)$ is a left jet bialgebroid, and $I:=\operatorname{Ker}\left(\partial_{J}{ }_{(L)}\right)$, we define

$$
K^{\star}:=\left\{u \in K^{*} \mid u\left(I^{n}\right)=0 \forall n \gg 0\right\}, \quad K_{\star}:=\left\{u \in K_{*} \mid u\left(I^{n}\right)=0 \forall n \gg 0\right\}
$$

It should be clear by the very definition that, in the first case, ${ }^{\star} K$, resp. ${ }_{\star} K$, is nothing but the subset of those functions in ${ }^{*} K$, resp. in ${ }_{*} K$, which are continuous with respect to the $I$-adic topology in ${ }^{*} K$, resp. in ${ }_{*} K$, and the discrete topology in $A$. Similarly for $K^{\star}$ and $K_{\star}$ in the second case. The key reason of interest for these objects lies in the following, well-known result:

Theorem 3.5.7. Let L be a Lie-Rinehart algebra which, as an A-module, is finite projective.

(a) Consider the right bialgebroid $J^{r}(L):=V^{\ell}(L)^{*}$. Then ${ }_{\star} J^{r}(L)$, as a left bialgebroid, is isomorphic to $V^{\ell}(L)$ : more precisely, the canonical map $V^{\ell}(L) \longrightarrow{ }_{\star}\left(V^{\ell}(L)^{*}\right)={ }_{\star} J^{r}(L)$ given by evaluation is an isomorphism of left bialgebroids.

Similarly, replacing $J^{r}(L):=V^{\ell}(L)^{*}$ with the right bialgebroid $V^{\ell}(L)_{*}$ one has a corresponding isomorphism of left bialgebroids $V^{\ell}(L) \longrightarrow{ }^{\star}\left(V^{\ell}(L)_{*}\right)$ still given by evaluation.

(b) Consider the left bialgebroid $J^{\ell}(L):={ }_{*} V^{r}(L)$. Then $J^{\ell}(L)^{\star}$, as a right bialgebroid, is isomorphic to $V^{r}(L)$ : more precisely, the canonical map $V^{r}(L) \longrightarrow\left({ }_{*} V^{r}(L)\right)^{\star}=J^{\ell}(L)^{\star}$ given by evaluation is an isomorphism of right bialgebroids.

Similarly, replacing $J^{\ell}(L):={ }_{*} V^{r}(L)$ with the left bialgebroid ${ }^{*} V^{r}(L)$ one has a corresponding isomorphism of right bialgebroids $V^{r}(L) \longrightarrow\left({ }^{*} V^{r}(L)\right)_{\star}$ still given by evaluation.

Remark 3.5.8. The standard isomorphism between $J^{r}(L):=V^{\ell}(L)^{*}$ and $J^{r}(L)_{\text {coop }}$ $=V^{\ell}(L)_{*}$ (see Remarks 3.5.2(b)) induces an isomorphism ${ }^{\star}\left(\left(V^{\ell}(L)^{*}\right) \cong V^{\ell}(L)\right.$. Similarly, we have also an analogous isomorphism $\left({ }_{*} V^{r}(L)\right)_{\star} \cong V^{r}(L)$.

Remark 3.5.9. Let $L$ be a finite projective Lie-Rinehart algebra and $Q$ be a (finite projective) $A$-module such that $L \oplus Q=F$ is a finite rank free $A$-module. We resume notation of \$2.1.6: so we take an $A$-basis $\left\{b_{1}, \ldots, b_{n}\right\}$ of $F$, and we set 
$Y=k b_{1} \oplus \cdots \oplus k b_{n}$, so that $F=A \otimes_{k} Y$; moreover, $L_{Q}=L \oplus\left(A \otimes_{k} Z\right)$ is a Lie-Rinehart algebra with $Z=Y \oplus Y \oplus Y \oplus \cdots$. One has $S(Y)^{\otimes \infty}:=S(Z)=$ $S(Y) \otimes S(Y) \otimes \cdots$ (recall that elements of an infinite tensor product of algebras are sums of tensor products with only finitely many factors different from 1). For $T \in\{Y, Z\}$, we let $\epsilon: S(T) \longrightarrow k-$ the counit map of $S(T)-$ be the unique $k$-algebra morphism given by $S(t):=0$ for $t \in T$, and we set $S(T)^{+}:=\operatorname{Ker}(\epsilon)$.

For any $n$, denote by $J_{f, n}^{r}\left(L_{Q}\right) \equiv V^{\ell}\left(L_{Q}\right)_{f, n}^{*}$ the subset of $V^{\ell}\left(L_{Q}\right)^{*}$ whose elements are all the $\lambda \in V^{\ell}\left(L_{Q}\right)^{*}$ such that $\left.\lambda\right|_{V^{\ell}(L) \otimes S(Y)^{\otimes n} \otimes S(Z)^{+}}=0$ and set $J_{f}^{r}\left(L_{Q}\right) \equiv V^{\ell}\left(L_{Q}\right)_{f}^{*}:=\bigcup_{n \in \mathbb{N}} J_{f, n}^{r}\left(L_{Q}\right)$. Then one can describe $J_{f, n}^{r}\left(L_{Q}\right)$ as $J_{f, n}^{r}\left(L_{Q}\right)=J^{r}(L) \widetilde{\otimes} \widetilde{S}\left(Y^{*}\right)^{\otimes^{n}} \widetilde{\otimes} 1 \widetilde{\otimes} 1 \widetilde{\otimes} \cdots$, where $\widetilde{S}\left(Y^{*}\right)$ denotes the completion of $S\left(Y^{*}\right)$ with respect to the weak topology; so we have also

$$
J_{f}^{r}\left(L_{Q}\right) \cong \sum_{n \in \mathbb{N}} J_{f, n}^{r}\left(L_{Q}\right)=\sum_{n \in \mathbb{N}} J^{r}(L) \widetilde{\otimes} \widetilde{S}\left(Y^{*}\right)^{\widetilde{\otimes}^{n}} \widetilde{\otimes} 1 \widetilde{\otimes} 1 \widetilde{\otimes} \cdots
$$

This $J_{f}^{r}\left(L_{Q}\right)$ is a sub-bialgebroid of $J^{r}\left(L_{Q}\right)$ : indeed, its right bialgebroid structure is described by

$$
\begin{gathered}
s_{r}: A \longrightarrow J_{f}^{r}\left(L_{Q}\right), \quad a \mapsto s_{r}(a) \otimes 1, \quad t_{r}: A \longrightarrow J_{f}^{r}\left(L_{Q}\right), \quad a \mapsto t_{r}(a) \otimes 1 \\
(\phi \otimes s)\left(\phi^{\prime} \otimes s^{\prime}\right):=\phi \phi^{\prime} \otimes s s^{\prime}, \quad \Delta(\phi \otimes s):=\left(\phi_{(1)} \otimes s_{(1)}\right) \otimes\left(\phi_{(2)} \otimes s_{(2)}\right), \\
\partial(\phi \otimes s):=\partial(\phi) \epsilon(s)
\end{gathered}
$$

for all $a \in A, \phi, \phi^{\prime} \in J^{r}(L), s, s^{\prime} \in \sum_{n \in \mathbb{N}} \widetilde{S}\left(Y^{*}\right)^{\widetilde{\otimes}^{n}} \widetilde{\otimes} 1 \widetilde{\otimes} 1 \widetilde{\otimes} \cdots(n \in \mathbb{N})$.

Last, let ${ }^{{ }} J_{f}^{r}\left(L_{Q}\right)$ be the subset of all $\delta \in{ }^{\star} J_{f}^{r}\left(L_{Q}\right)$ such that

$$
\left.\delta\right|_{J^{r}(L) \widetilde{\otimes} S\left(Y^{*}\right)^{\otimes n} \widetilde{\otimes} S\left(Z^{*}\right)^{+}}=0
$$

for $n \gg 0$. It is easy to see that ${ }^{\star}{ }_{f} J_{f}^{r}\left(L_{Q}\right)$ is a left sub-bialgebroid of ${ }^{\star} J_{f}^{r}\left(L_{Q}\right)$, isomorphic to $V^{\ell}\left(L_{Q}\right)$.

\section{Quantum groupoids}

In this section we introduce quantum groupoids - i.e. topological bialgebroids which are formal deformations of those attached to Lie-Rinehart algebras. Then we show that taking suitable "(linear) duals" we get an antiequivalence among the categories of objects of these two types.

4.0.10. The $h$-adic topology. If $V$ is any $k[[h]]-$ module, it is endowed with the following decreasing filtration: $V \supseteq h V \supseteq h^{2} V \supseteq \cdots \supseteq h^{n} V \supseteq h^{n+1} V \supseteq \cdots \supseteq$. Then $V$ is also endowed with the $h$-adic topology, which is the unique one for which $V$ is a topological $k[[h]]$-module in which $\left\{h^{m} V\right\}_{m \in \mathbb{N}}$ is a basis of neighborhoods 
of 0 . Indeed, $V$ is then a pseudo-metric space, as the $h$-adic topology is the one induced by the following pseudo-metric:

$$
\begin{array}{r}
d(x, y):=\|x-y\|=2^{-m} \text { with } m:=\sup \left\{s \in \mathbb{N} \mid(x-y) \in h^{s} V\right\} \\
\forall x, y \in V .
\end{array}
$$

The topological space $V$ is Hausdorff if and only if the pseudo-metric $d$ is a metric: in turn, this occurs if and only if $\bigcap_{m \in \mathbb{N}} h^{m} V=\{0\}$, which means that each point in $V$ forms a closed subset.

4.1. Quantum groupoids. In this subsection we introduce the notion of "quantum groupoids": these are special "quantum bialgebroids", namely (topological) bialgebroids which are formal deformations of those of type $V^{\ell}(L), V^{r}(L), J^{r}(L)$ or $J^{\ell}(L)$. We begin with the ones associated with the first two cases:

Definition 4.1.1. A left quantum universal enveloping algebroid (=LQUEAd) is a topological left bialgebroid $\left(H_{h}, A_{h}, s_{h}^{\ell}, t_{h}^{\ell}, m_{h}, \Delta_{h}, \epsilon_{h}\right)$ over a topological $k[[h]]-$ algebra $A_{h}$ such that:

(i) $A_{h}$ is isomorphic to $A[[h]]$ as a topological $k[[h]]$-module, for some $k-$ algebra $A$, and this isomorphism induces an algebra isomorphism $A_{h} / h A_{h} \cong$ $A[[h]] / h A[[h]] \cong A$;

(ii) $H_{h}$ is isomorphic to $V^{\ell}(L)[[h]]$ as a topological $k[[h]]$-module where $V^{\ell}(L)$ is the left bialgebroid associated with some Lie-Rinehart $A$-algebra $L$, as in $\S 3.2 .9$;

(iii) $H_{h} / h H_{h} \cong V^{\ell}(L)[[h]] / h V^{\ell}(L)[[h]]$ is isomorphic to $V^{\ell}(L)$ as a left $A$ bialgebroid via the isomorphism $A_{h} / h A_{h} \cong A[[h]] / h A[[h]] \cong A$ mentioned in (i);

(iv) denote by $H_{h \triangleleft} \widehat{\Theta}_{A_{h}}{ }_{\triangleright} H_{h}$ the completion of $H_{h \triangleleft}{\underset{A}{A_{h}}}_{\triangleright} H_{h}$ with respect to the $h$-adic topology, and define the ( $h$-adically completed) Takeuchi product as

$$
\begin{aligned}
& H_{h \triangleleft} \underset{A_{h}}{\widehat{x}} H_{h}:= \\
& \quad\left\{\sum_{i} u_{i} \otimes u_{i}^{\prime} \in H_{h \triangleleft} \widehat{\otimes}_{A_{h}} \triangleright H_{h} \mid \sum_{i}\left(a \triangleright u_{i}\right) \otimes u_{i}^{\prime}=\sum_{i} u_{i} \otimes\left(u_{i}^{\prime} \triangleleft a\right)\right\}
\end{aligned}
$$

then the coproduct $\Delta_{h}$ of $H_{h}$ takes values in $H_{h \triangleleft} \widehat{A}_{A_{h}} \widehat{Q}_{\triangleright} H_{h}$.

In this setting, we shall say that $H_{h}$ is a quantization, or a quantum deformation, of $V^{\ell}(L)$; we shall resume it in short using notation $V^{\ell}(L)_{h}:=H_{h}$.

In a similar, parallel way, we define the notion of right quantum universal enveloping algebroid (=RQUEAd) as well, just replacing "left" with "right" and $V^{\ell}(L)$ with $V^{r}(L)$, cf. §3.3.3. 
We define morphisms among left, resp. right, quantum universal enveloping algebroids like in Definition 3.2.3; moreover, we use notation (LQUEAd), resp. RQUEAd, to denote the category of all left, resp. right, quantum universal enveloping algebroids. If $A_{h}$ is a fixed ground $k[[h]]-$ algebra, then we write (LQUEAd) $A_{h}$, resp. (RQUEAd) $A_{h}$, to denote the subcategory - in (LQUEAd), resp. (RQUEAd) - whose objects are all the left, resp. right, quantum universal enveloping algebroids over $A_{h}$, and whose morphisms are selected as in Definition 3.2.3.

Remarks 4.1.2. (a) $U$ is a LQUEAd $\Longleftrightarrow U_{c o o p}^{o p}$ is a RQUEAd $\Longleftrightarrow U^{o p}$ is a RQUEAd.

(b) If $\left(V^{\ell}(L)_{h}, A_{h}, s_{h}^{\ell}, t_{h}^{\ell}, m_{h}, \Delta_{h}, \epsilon_{h}\right)$ is any LQUEAd, then $A_{h}$ is a deformation of the algebra $A$ : then, as usual, one can define a Poisson structure on the base algebra $A$ as follows:

$$
\{f, g\}:=\frac{f^{\prime} *_{h} g^{\prime}-g^{\prime} *_{h} f^{\prime}}{h} \bmod h A_{h} \quad \forall f, g \in A
$$

where $f^{\prime} \in A_{h}$ and $g^{\prime} \in A_{h}$ are such that $f^{\prime} \bmod h A_{h}=f$ and $g^{\prime} \bmod h A_{h}=g$. The same observations makes sense if one has to do with a RQUEAd $V^{r}(L)_{h}$.

(c) The definitions given so far make sense for any Lie-Rinehart algebra $L$. However, from now on we shall assume in addition that $L$, as an A-module, is finitely generated projective.

The following theorem is proved in [34] (Theorem 5.16):

Theorem 4.1.3. Let $\left(V^{\ell}(L)_{h}, A_{h}, s_{h}^{\ell}, t_{h}^{\ell}, m_{h}, \Delta_{h}, \epsilon_{h}\right)$ be a LQUEAd. Define

$$
\begin{aligned}
\delta(a) & :=\frac{t_{h}^{\ell}\left(a^{\prime}\right)-s_{h}^{\ell}\left(a^{\prime}\right)}{h} \bmod h V^{\ell}(L)_{h} & \forall a \in A \\
\delta(X) & :=\Delta^{[1]}(X)_{2,1}-\Delta^{[1]}(X) \in V^{\ell}(L) \underset{A}{\otimes} V^{\ell}(L) & \forall X \in L \\
\text { with } & \Delta^{[1]}(X) & :=\frac{\Delta_{h}\left(X^{\prime}\right)-X^{\prime} \otimes 1-1 \otimes X^{\prime}}{h} \bmod h\left(V^{\ell}(L)_{h} \widehat{\otimes} \hat{A}_{h} V^{\ell}(L)_{h}\right) \\
\text { and } & \Delta^{[1]}(X)_{2,1} & :=\sum_{[X]} X_{[2]} \otimes X_{[1]} \quad \text { if } \quad \Delta^{[1]}(X)=\sum_{[X]} X_{[1]} \otimes X_{[2]}
\end{aligned}
$$

where $X^{\prime} \in V^{\ell}(L)_{h}$ is any lift of $X$ (i.e. $\left.X^{\prime} \bmod h V^{\ell}(L)_{h}=X\right)$ and $a^{\prime} \in A_{h}$ is any lift of $a$.

Then $\delta(a) \in L$ and $\delta(X) \in \bigwedge_{A}^{2} L$; this gives to $L$ the structure of a Lie-Rinehart bialgebra. Also, the Poisson structure on A induced by this Lie-Rinehart bialgebra (cf. Remarks 2.2.2 (c)) coincides with the one obtained as the classical limit of the base*-algebra $A_{h}$ (cf. Remarks 4.1.2 (b)).

Remark 4.1.4. In the above statement, we took formulas opposite to those in [34]: indeed, this allows us to deduce the very last claim. 
Example 4.1.5. (cf. [34]) Let $P$ be a smooth manifold, $D$ the algebra of global differential operators on $P$ and $A:=\mathcal{C}^{\infty}(P)$. Let $D[[h]]$ be the trivial deformation of $D$. Let

$$
\mathcal{F}=1 \otimes 1+h B_{1}+\cdots \in\left(D \otimes_{A} D\right)[[h]] \cong D[[h]] \widehat{\otimes}_{A[[h]]} D[[h]]
$$

be a formal series of bidifferential operators. It is easy to see that $\mathcal{F}$ is a twistor (cf. Definition 3.2.7) iff the multiplication on $A[[h]]$ defined by $f *_{h} g=\mathcal{F}(f, g)$ for all $f, g \in A[[h]]$, is associative, with identity being the constant function 1, i.e. iff $*_{h}$ is a star product on $P$. The twisted bialgebroid structure on $D_{h}:=D[[h]]$ can be easily described: $A_{h}=A[[h]]$ has the star product defined above, $s_{h}^{\ell}: A_{h} \longrightarrow D_{h}$ and $t_{h}^{\ell}: A_{h} \longrightarrow D_{h}$ are given by $s_{h}^{\ell}(f) g=f *_{h} g, t_{h}^{\ell}(f) g=g *_{h} f$, for $f$, $g \in A$, the coproduct $\Delta_{h}: D_{h} \longrightarrow D_{h} \widehat{\otimes}_{A_{h}} D_{h}$ is $\Delta_{h}(x):=\mathcal{F}^{\#-1}(\Delta(x) \cdot \mathcal{F})$ for $x \in D_{h}$.

In Section 7 later on we shall explicitly provide a specific example of this kind.

Theorem 4.1.3 has a natural counterpart for RQUEAd's as follows:

Theorem 4.1.6. Let $\left(V^{r}(L)_{h}, A_{h}, s_{h}^{r}, t_{h}^{r}, m_{h}, \Delta_{h}, \epsilon_{h}\right)$ be a RQUEAd. Define

$$
\begin{array}{rlrl}
\delta(a) & :=\frac{s_{h}^{r}(a)-t_{h}^{r}(a)}{h} \bmod h V^{r}(L)_{h} & \forall a \in A \\
\delta(X) & :=\Delta^{[1]}(X)_{2,1}-\Delta^{[1]}(X) \in V^{r}(L) \underset{A}{\otimes} V^{r}(L) \quad \forall X \in L \\
\text { with } \quad \Delta^{[1]}(X) & :=\frac{\Delta_{h}\left(X^{\prime}\right)-X^{\prime} \otimes 1-1 \otimes X^{\prime}}{h} \bmod h\left(V^{r}(L)_{h} \widehat{\otimes} \widehat{A}_{h} V^{r}(L)_{h}\right) \\
\text { and } \quad \Delta^{[1]}(X)_{2,1} & :=\sum_{[X]} X_{[2]} \otimes X_{[1]} \quad \text { if } \quad \Delta^{[1]}(X)=\sum_{[X]} X_{[1]} \otimes X_{[2]}
\end{array}
$$

where $X^{\prime} \in V^{r}(L)_{h}$ is any lift of $X$ (i.e. $\left.X^{\prime} \bmod h V^{r}(L)_{h}=X\right)$ and $a^{\prime} \in A_{h}$ is any lift of $a$.

Then $\delta(a) \in L$ and $\delta(X) \in \bigwedge_{A}^{2} L$; this gives to $L$ the structure of a LieRinehart bialgebra. Moreover, the Poisson structure induced on A by this LieRinehart bialgebra structure is opposite to the one obtained as the classical limit of the base *-algebra $A_{h}$ (cf. Remarks 4.1.2 (b)).

Remark 4.1.7. The previous result can be proved just like Theorem 4.1.3 in [34]. Otherwise, one can deduce Theorem 4.1.6 from Theorem 4.1.3 applied to $V^{\ell}(L)_{h}:=V^{r}(L)_{h}^{o p}$, which is a LQUEAd - cf. Remarks 4.1.2 (a). In particular, the Lie-Rinehart bialgebra structure induced on $L$ by the RQUEAd $V^{r}(L)_{h}$ is opposite to that induced by the LQUEAd $V^{\ell}(L)_{h}:=V^{r}(L)_{h}^{o p}$.

We introduce now a second type of "quantum bialgebroids", namely quantizations of jets: 
Definition 4.1.8. A right quantum formal series algebroid (=RQFSAd) is a topological right bialgebroid $\left(K_{h}, A_{h}, s_{h}^{r}, t_{h}^{r}, m_{h}, \Delta_{h}, \partial_{h}\right)$ over a topological $k[[h]]$-algebra $A_{h}$ such that:

(i) $A_{h}$ is isomorphic to $A[[h]]$ as a topological $k[[h]]$-module, for some $k-$ algebra $A$, and this isomorphism induces an algebra isomorphism $A_{h} / h A_{h} \cong$ $A[[h]] / h A[[h]] \cong A$;

(ii) $K_{h}$ is isomorphic to $J^{r}(L)[[h]]$ as a topological $k[[h]]$-module where $J^{r}(L)$ is the right bialgebroid of jets associated with some finite projective Lie-Rinehart $A$-algebra $L$ as in $\S 3.5 .1$;

(iii) $K_{h} / h K_{h} \cong J^{r}(L)[[h]] / h J^{r}(L)[[h]]$ is isomorphic to $J^{r}(L)$ as a right $A-$ bialgebroid via the isomorphism $A_{h} / h A_{h} \cong A[[h]] / h A[[h]] \cong A$ mentioned in (i);

(iv) letting $I_{h}:=\left\{\psi \in K_{h} \mid \partial(\psi) \in h A_{h}\right\}$ — which is easily seen to be a twosided ideal in $K_{h}$ - we have that $K_{h}$ is complete in the $I_{h}$-adic topology;

(v) denote by $K_{h} \underset{A_{h}}{\mathbb{\otimes}} \checkmark K_{h}$ the completion of $K_{h} \underset{A_{h}}{\otimes} \mathbb{V}_{h}$ with respect to the topology defined by the filtration $\left(\sum_{p+q=n} I_{h}^{p} \otimes I_{h}^{q}\right)_{n \in \mathbb{N}} ;$ also, define the Takeuchi product as

$K_{h \triangleleft} \underset{A_{h}}{\widetilde{\times}} \triangleright K_{h}:=\left\{\sum_{i} u_{i} \otimes u_{i}^{\prime} \in K_{h} \underset{A_{h}}{\widetilde{\otimes}} K_{h} \mid \sum_{i}\left(a \triangleright u_{i}\right) \otimes u_{i}^{\prime}=\sum_{i} u_{i} \otimes\left(u_{i}^{\prime} \triangleleft a\right)\right\}$

then the coproduct $\Delta_{h}$ of $K_{h}$ takes values in $K_{h \triangleleft} \underset{A_{h}}{\widetilde{\times}}, K_{h}$.

In this setting, we shall say that $K_{h}$ is a quantization, or a quantum deformation, of $J^{r}(L)$; we shall resume it in short using notation $J^{r}(L)_{h}:=K_{h}$.

In a similar, parallel way, we define the notion of left formal series algebroid (=LQFSAd) as well, just replacing "left" with "right" and $J^{r}(L)$ with $J^{\ell}(L)$.

We define morphisms among right, resp. left, quantum formal series algebroids like in Definition 3.2.3; moreover, we use notation (RQFSAd), resp. LQFSAd, to denote the category of all right, resp. left, quantum formal series algebroids. If $A_{h}$ is a fixed ground (topological) $k[[h]]-$ algebra, then we write (RQFSAd) $A_{h}$, resp. (LQFSAd) $A_{h}$, to denote the subcategory - in (RQFSAd), resp. (LQFSAd) whose objects are all the right, resp. left, quantum formal series algebroids over $A_{h}$, and whose morphisms are selected as in Definition 3.2.3.

Remarks 4.1.9. (a) From the analysis in $\S 3.5 .5$ we can argue that one could define a RQFSAd also as a deformation of the right bialgebroid $V^{\ell}(L)_{*}$, and a LQFSAd as a deformation of the left bialgebroid ${ }^{*} V^{r}(L)$. On the other hand, the very conclusion of §3.5.5 itself also tells us that it is enough to consider the notions of RQFSAd and LQFSAd introduced in Definition 4.1.8 above.

(b) $K_{h}$ is a LQFSAd $\Longleftrightarrow\left(K_{h}^{o p}\right)_{\text {coop }}$ is a RQFSAd $\Longleftrightarrow K_{h}^{o p}$ is a RQFSAd.

4.1.10. Further "half Hopf" structures on quantum groupoids. By construction, our quantum groupoids are just (left or right) bialgebroids, namely deformations of 
such (left or right) bialgebroids as $V^{\ell / r}(L)$ and $J^{\ell / r}(L)$. However, $V^{\ell / r}(L)$ and $J^{\ell / r}(L)$ actually bear further structure, which "automatically inheriteld" by their quantizations too. To explain it, we fix some terminology.

Let

$$
\checkmark U \underset{A^{o p}}{\otimes} U_{\triangleleft}:=U \underset{k}{\otimes} U / \operatorname{Span}\left(\{(a \triangleright u) \otimes v-u \otimes(v \triangleleft a)\}_{u, v \in U}^{a \in A}\right)
$$

for some left bialgebroid $U$ over $A$; then define a "Hopf-Galois" map

$$
\checkmark U \underset{A^{o p}}{\otimes} U_{\triangleleft} \stackrel{\alpha_{\ell}}{\longrightarrow} U_{\triangleleft} \underset{A}{\otimes} U, \quad u \otimes v \mapsto u_{(1)} \otimes u_{(2)} v .
$$

Similarly, one can consider an analogous tensor product ${ }_{\triangleright} U \underset{A^{o p}}{\otimes} U_{\triangleleft}$ and a corresponding "Hopf-Galois" map $U_{A^{o p}} U_{\triangleleft} \stackrel{\alpha_{r}}{\longrightarrow} U_{\triangleleft} \otimes_{A} U, u \otimes v \mapsto v_{(1)} u \otimes v_{(2)}$. On the other hand, for a right bialgebroid $W$ over $B$ one consider suitable tensor products

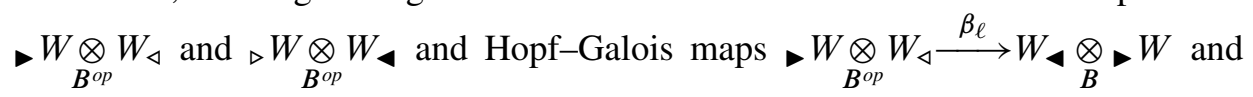
$\triangleright W_{B^{o p}}^{\otimes} W_{\triangleleft} \stackrel{\beta_{r}}{\longrightarrow} W_{\triangleleft} \underset{B}{\otimes} W$ involving them. Then $U$ is called a left, resp. a right, Hopf left bialgebroid iff the map $\alpha_{\ell}$, resp. $\alpha_{r}$, is a bijection; similarly, $W$ is called a left Hopf right bialgebroid, respectively a left, resp. a right, Hopf right bialgebroid, iff the map $\beta_{\ell}$, resp. $\beta_{r}$, is a bijection (cf. [4], [22], [24], [23]).

It is known that $V^{\ell}(L)$, resp. $V^{r}(L)$, is both a left and right Hopf left, resp. right, bialgebroid. The same holds for $J^{\ell}(L)$ and $J^{r}(L)$ too - actually because they have even stronger properties, namely they are Hopf algebroids, in the sense of Böhm-Szlachányi (cf. [5], [3], [25], [19], [22]).

Any quantum groupoid has its own Hopf-Galois maps, whose semiclassical specialization are the analogous maps for its semiclassical limit: e.g., the HopfGalois map $\alpha_{\ell}$ for any $V^{\ell}(L)_{h}$ yields by specialization the same name HopfGalois map for $V^{\ell}(L)$. The latter map is bijective (since $V^{\ell}(L)$ is a left Hopf left bialgebroid) hence, by a standard argument, its deformation - the map $\alpha_{\ell}$ for $V^{\ell}(L)_{h}$ - is bijective too: thus in turn $V^{\ell}(L)_{h}$ is a left Hopf left bialgebroid as well! With similar reasonings, looking all types of quantum groupoids we find that any $V^{\ell}(L)_{h}$ and any $J^{\ell}(L)_{h}$ are both right and left Hopf left bialgebroids, while any $V^{r}(L)_{h}$ and any $J^{r}(L)_{h}$ are both left and right Hopf right bialgebroids.

4.1.11. Liftings in a $(\mathbf{R} / \mathbf{L}) \mathbf{Q F S A d}$. Let $L$ be a Lie-Rinehart algebra which is finite projective (as an $A$-module). Set $\mathfrak{J}_{J} r_{(L)}:=\operatorname{Ker}\left(\partial_{J^{r}(L)}\right)$ : then $\mathfrak{J}_{J^{r}(L)} / \mathfrak{J}_{J^{r}(L)}^{2} \cong L^{*}$ as $A$-modules, by definitions. Given $\Phi \in L^{*}$, we shall call a lift of $\Phi$ in $J^{r}(L)$ any $\phi \in \mathfrak{J}_{J^{r}(L)}$ such that through the above isomorphism one has $\phi \bmod \mathfrak{J}_{J^{r}(L)}^{2}=\Phi$. Now let $K_{h}=J^{r}(L)_{h}$ be a RQFSAd, deformation of $J^{r}(L)$. For any $\Phi \in L^{*}$, we shall call a lift of $\Phi$ in $J^{r}(L)_{h}$ any element $\phi^{\prime} \in J^{r}(L)_{h}$ such that $\phi^{\prime} \bmod h J^{r}(L)_{h}$ is a lift of $\Phi$ in $J^{r}(L)$. In short, this means $\Phi \equiv\left(\phi^{\prime} \bmod h J^{r}(L)_{h}\right) \bmod \mathfrak{J}_{J^{r}(L)}^{2}$. 
Also, if $a \in A$ we call a lift of $a$ in $A_{h}$ any $a^{\prime} \in A_{h}$ such that $a^{\prime} \bmod h A_{h}=a$.

Changing "right" into "left", similar remarks and terminology apply for defining "lifts" of elements of $J^{\ell}(L)$ in some associated LQFSAd, say $J^{\ell}(L)_{h}$.

The next result introduces semiclassical structures induced on a Lie-Rinehart algebra $L$ by quantizations of the form $J^{r}(L)$ or $J^{\ell}(L)$. Indeed, this is the dual counterpart of Theorem 4.1.3.

Theorem 4.1.12. Let $J^{r}(L)_{h}$ be a RQFSAd, namely a deformation of $J^{r}(L)$ as above. Then L inherits from this quantization a structure of Lie-Rinehart bialgebra, namely the unique one such that the Lie bracket and the anchor map of $L^{*}$ are given (notation as above) by

$$
\begin{aligned}
{[\Phi, \Psi] } & :=\left(\frac{\phi^{\prime} \psi^{\prime}-\psi^{\prime} \phi^{\prime}}{h} \bmod h J^{r}(L)_{h}\right) \bmod \mathfrak{J}_{J^{r}(L)}^{2} \\
\omega(\Phi)(a) & :=\left(\frac{\phi^{\prime} r\left(a^{\prime}\right)-r\left(a^{\prime}\right) \phi^{\prime}}{h} \bmod h J^{r}(L)_{h}\right) \bmod \mathfrak{J}_{J^{r}(L)} \\
& =\partial\left(\frac{\phi^{\prime} r\left(a^{\prime}\right)-r\left(a^{\prime}\right) \phi^{\prime}}{h}\right) \bmod h A_{h}
\end{aligned}
$$

for all $\Phi, \Psi \in L^{*}$ and $a \in A$, where $\phi^{\prime}$ and $\psi^{\prime}$ are liftings in $J^{r}(L)_{h}$ of $\Phi$ and $\Psi$ respectively, $a^{\prime}$ is a lifting in $A_{h}$ of $a \in A$, and finally $r\left(a^{\prime}\right)$ stands for either $s_{h}^{r}\left(a^{\prime}\right)$ or $t_{h}^{r}\left(a^{\prime}\right)$.

Proof. First, it is easy to see that the maps [, ] and $\omega$ as given in the statement are well-defined, i.e. they do not depend on the choice of liftings, nor of the choice of either of $s_{h}^{r}\left(a^{\prime}\right)$ or $t_{h}^{r}\left(a^{\prime}\right)$ acting as $r\left(a^{\prime}\right)$. Moreover, by construction we have $[\Phi, \Psi] \in \mathfrak{J}_{J^{r}(L)} / \mathfrak{J}_{J^{r}(L)}^{2} \cong L^{*}$. Also, again by construction we have $\omega(\Phi)(a) \in$ $J^{r}(L) / \mathfrak{J}_{J^{r}(L)}$; now the latter space identifies with $\partial\left(J^{r}(L)\right)=A$, thus $\omega(\Phi)(a) \in$ $A$ via these identifications, so that $\omega(\Phi)$ is a $k$-linear endomorphism of $A$.

Now, the definition of both [, ] and $\omega$ is made via a commutator in $J^{r}(L)_{h}$. As the commutator - in any associative $k$-algebra - is a $k$-bilinear Lie bracket and satisfies the Leibniz identity (involving the associative product), one can easily argue at once from definitions that $L^{*}$ with the given bracket and anchor map is indeed a Lie-Rinehart algebra (over $A$ ).

What is more demanding is to prove that with this structure the pair $\left(L, L^{*}\right)$ of Lie-Rinehart $A$-algebras fulfills all constraints to be a Lie-Rinehart bialgebra. Indeed, we shall not provide a direct proof for that: instead, we have recourse to a duality argument, using the notions and results of Subsec. 5.1 later on. Indeed, there we shall see that ${ }_{\star} J^{r}(L)_{h}$ is a LQUEAd, hence by Theorem 4.1.3 we know that $\left(L, L^{*}\right)$ is a Lie-Rinehart bialgebra. 
The analogue of Theorem 4.1.12 for LQFSAd's (with essentially the same proof) is the following:

Theorem 4.1.13. Let $J^{\ell}(L)_{h}$ be a LQFSAd, namely a deformation of $J^{\ell}(L)$. Then $L$ inherits from this quantization a structure of Lie-Rinehart bialgebra, namely the unique one for which the Lie bracket and the anchor map of $L^{*}$ are given (notation as above) by

$$
\begin{aligned}
{[\Phi, \Psi] } & :=\left(\frac{\phi^{\prime} \psi^{\prime}-\psi^{\prime} \phi^{\prime}}{h} \bmod h J^{\ell}(L)_{h}\right) \bmod \mathfrak{J}_{J^{r}(L)}^{2} \\
\omega(\Phi)(a) & :=\left(\frac{\phi^{\prime} r\left(a^{\prime}\right)-r\left(a^{\prime}\right) \phi^{\prime}}{h} \bmod h J^{\ell}(L)_{h}\right) \bmod \mathfrak{J}_{J^{r}(L)}
\end{aligned}
$$

for all $\Phi, \Psi \in L^{*}$ and $a \in A$, where $\phi^{\prime}$ and $\psi^{\prime}$ are liftings in $J^{\ell}(L)_{h}$ of $\Phi$ and $\Psi$ respectively, $a^{\prime}$ is a lifting in $A_{h}$ of $a \in A$, and finally $r\left(a^{\prime}\right)$ stands for either $s_{h}^{r}\left(a^{\prime}\right)$ ort $t_{h}^{r}\left(a^{\prime}\right)$.

Remark 4.1.14. The result above can be proved like its analogue for RQFSAd's, i.e. Theorem 4.1.12. Otherwise, one can get the former from the latter applied to $J^{r}(L)_{h}:=J^{\ell}(L)_{h}^{o p}$, which is a RQFSAd - cf. Remarks 4.1.9(c). In particular, the Lie-Rinehart bialgebra structure induced on $L$ by the LQFSAd $J^{\ell}(L)_{h}$ is oppositecoopposite to that induced by the RQFSAd $J^{r}(L)_{h}:=J^{\ell}(L)_{h}^{o p}$.

4.2. Extending quantizations: from the finite projective to the free case. Let $L$ be a Lie-Rinehart algebra over $A$ which is finite projective as an $A$-module. With the procedure presented in $\$ 2.1 .6$, we can find a projective $A$-module $Q$ (a complement of $L$ in a finite free $A$-module $F$ ) and use it to build a new Lie-Rinehart algebra $L_{Q}:=L \oplus(Q \oplus L \oplus Q \oplus L \oplus \cdots)=L \oplus R$, which as an $A$-module is free. Then we fix an $A$-basis $\left\{b_{1}, \ldots, b_{n}\right\}$ of $F$ from which we construct a good basis $\left\{e_{i}\right\}_{i \in T}$ of $L_{Q}$ and a good basis $\left\{v_{t}\right\}_{t \in T}$ of $R$. Set $Y:=\bigoplus_{i=1}^{n} k b_{i}$ so that $F=A \otimes_{k} Y$, $T:=\mathbb{N} \times\{1, \ldots, n\}, Z:=\underset{t \in T}{\oplus} k v_{t}$, hence $R=A \otimes_{k}(Y \oplus Y \oplus \cdots)=A \otimes_{k} Z$. Moreover, one has also $V^{\ell}\left(L_{Q}\right)=V^{\ell}(L) \otimes_{k} S(Z)$ with $S(Z)=S(Y) \otimes S(Y) \otimes \cdots$.

4.2.1. Extending QUEAd's. Let $L$ be a finite projective Lie-Rinehart algebra, for which we consider for it all the objects and constructions mentioned just above. Let $V^{\ell}(L)_{h} \in\left(\right.$ LQUEAd) $A_{h}$ be a (left) quantization of the left bialgebroid $V^{\ell}(L)$. Consider

$V^{\ell}(L)_{h, Y}:=h$-adic completion of $V^{\ell}(L)_{h} \otimes_{k} S(Y) \otimes_{k} S(Y) \otimes \cdots=: V^{\ell}(L)_{h} \widehat{\otimes}_{k} S(Z)$

In order to describe it, for $\underline{d} \in T^{(\mathbb{N})}$ we set $e^{\underline{d}}:=\prod_{t \in T} e_{t}^{\underline{d}(t)}$ and $\varpi(\underline{d}):=$ $\max \left\{\varpi\left(e_{t}\right) \mid \underline{d}(t) \neq 0\right\}$ (cf. Definition 2.1.7); 
Proposition 4.2.2. Any element of $V^{\ell}(L)_{h, Y}$ can be written in a unique way as

$$
\begin{array}{rlrl}
\sum_{\underline{d} \in T^{(\mathbb{N})}} t^{\ell}\left(a_{\underline{d}}\right) e^{\underline{d}} & =\lim _{n \rightarrow+\infty} \sum_{\substack{d \in T^{(\mathbb{N})} \\
|\underline{d}| \underline{+} \varpi(\underline{d}) \leq n}} t^{\ell}\left(a_{\underline{d}}\right) e^{\underline{d}} \\
\text { with } \quad & \lim _{|\underline{d}|+\infty(\underline{d}) \rightarrow+\infty}\left\|a_{\underline{d}}\right\| & =0 & \text { (notation of } \S \text { 4.0.10). }
\end{array}
$$

Proof. It is obvious that any element of the given lies in $V(L)_{h Y}$. Conversely, let $u \in V(L)_{h Y}$. Write $u=u_{0}+h u_{1}+\cdots+h^{n} u_{n}+\cdots$ with $u_{i} \in V\left(L_{Q}\right)$ for all $i$. Now, for all $i \in \mathbb{N}$, each $u_{i}$ can be written as $u_{i}=\sum_{\underline{\alpha} \in \mathbb{N}(T)} t^{\ell}\left(u_{i}^{\underline{\alpha}}\right) e^{\underline{\alpha}}$ where all but a finite number of the $u_{i}^{\underline{\alpha}}$,s are zero. Set $u_{\underline{\alpha}}:=\sum_{i} h^{i} u_{i}^{\underline{\alpha}}$, so $u=\sum_{\underline{\alpha}} u_{\underline{\alpha}} e^{\underline{\alpha}}$; we show that $\lim _{|\alpha|+\varpi(\underline{\alpha}) \rightarrow+\infty}\left\|u_{\underline{\alpha}}\right\|=0$. Pick $n_{0} \in \mathbb{N}$; choosing $A>\max \{|\underline{\alpha}|+\omega(\underline{\alpha}) \mid \exists i \leq$ $\left.n_{0}: u_{i}^{\underline{\alpha}} \neq 0\right\}$, for any $|\underline{\alpha}|+\omega(\underline{\alpha})>A$ we have $\left\|u_{\underline{\alpha}}\right\|<2^{-n_{0}}$.

Now, there exists a unique left bialgebroid structure on $V^{\ell}(L)_{h, Y}$ given as follows:

$$
\begin{aligned}
& t_{\ell}^{Y}: A_{h} \longrightarrow V^{\ell}(L)_{h, Y}, \quad a \mapsto t_{\ell}(a) \otimes 1, \quad s_{\ell}^{Y}: A_{h} \longrightarrow V^{\ell}(L)_{h, Y}, \quad a \mapsto s_{\ell}(a) \otimes 1 \\
& \Delta_{V^{\ell}(L)_{h, Y}}(a \otimes s):=\left(a_{(1)} \otimes s_{(1)}\right) \otimes\left(a_{(2)} \otimes s_{(2)}\right) \\
& \text { if } \Delta_{h}(a)=a_{(1)} \otimes a_{(2)}, \quad \Delta_{S(Z)}(s)=s_{(1)} \otimes s_{(2)} \\
& \epsilon_{h}(a \otimes s):=\epsilon_{h}(a) \epsilon(s), \quad(a \otimes s)\left(a^{\prime} \otimes s^{\prime}\right):=a a^{\prime} \otimes s s^{\prime}
\end{aligned}
$$

where the right-hand side factor map $\epsilon$ above is just the standard counit map $\epsilon: S(Z) \longrightarrow k$ of the Hopf $k$-algebra $S(Z)$, uniquely determined by $\epsilon(z)=0$ for every $z \in Z$. It is easy to see that

(a) $V^{\ell}(L)_{h, Y}$ is a quantization of the left bialgebroid $V^{\ell}\left(L_{Q}\right)$;

(b) $\pi_{Y}:=i d_{V^{\ell}(L)_{h, Y}} \widehat{\otimes}_{k} \epsilon: V^{\ell}(L)_{h, Y}:=V^{\ell}(L)_{h} \widehat{\otimes}_{k} S(Z) \rightarrow V^{\ell}(L)_{h}$ is an epimorphism of left bialgebroids.

A similar construction is possible if we take a RQUEAd $V^{r}(L)_{h}$ instead of the LQUEAd $V^{\ell}(L)_{h}$.

4.2.3. Extending QFSAd's. Let $L$ be a finite projective Lie-Rinehart algebra, and adopt again notations as before. Recall also that in Remark 3.5.9 we have introduced $J_{f}^{r}\left(L_{Q}\right):=V^{\ell}\left(L_{Q}\right)^{* f}$.

Let $J^{r}(L)_{h} \in(\mathrm{RQFSAd})_{A_{h}}$ be a quantization of $J^{r}(L)$. Keeping notation as in $\S 4.2 .1$, consider

$$
J^{r}(L)_{h, Y}:=h \text {-adic completion of } \sum_{n \in \mathbb{N}} J^{r}(L)_{h} \widetilde{\otimes}_{k} S\left(Y^{*}\right)^{\widetilde{\otimes}} \otimes 1 \otimes 1 \otimes 1 \cdots
$$


where $J^{r}(L)_{h} \widetilde{\otimes} S\left(Y^{*}\right)^{\widetilde{\otimes} n} \otimes 1 \otimes 1 \otimes \cdots$ is the $\left(\left(S\left(Y^{*}\right)^{\otimes n}\right)^{+} \otimes 1 \otimes 1 \otimes 1 \cdots\right)$-adic completion of $J^{r}(L)_{h} \otimes S\left(Y^{*}\right)^{\otimes n} \otimes 1 \otimes 1 \otimes 1 \cdots$. There exists a unique right bialgebroid structure on $J^{r}(L)_{h, Y}$

$$
\begin{gathered}
t_{r}^{Y}: A \longrightarrow J^{r}(L)_{h, Y}, \quad a \mapsto t_{r}(a) \otimes 1, \quad s_{r}^{Y}: A_{h} \longrightarrow J^{r}(L)_{h, Y}, \quad a \mapsto s_{r}(a) \otimes 1 \\
(a \otimes s)\left(a^{\prime} \otimes s^{\prime}\right):=a a^{\prime} \otimes s s^{\prime}, \quad \Delta(a \otimes s):=\left(a_{(1)} \otimes s_{(1)}\right) \otimes\left(a_{(2)} \otimes s_{(2)}\right), \\
\partial_{h}(a \otimes s):=\partial_{h}(a) \epsilon^{*}(s)
\end{gathered}
$$

Then one easily sees that

(a) $J^{r}(L)_{h, Y}$ is a quantization of the right bialgebroid $J_{f}^{r}\left(L_{Q}\right)$;

(b) $\pi^{Y}:=i d_{J^{r}(L)_{h, Y}} \otimes_{k} \epsilon^{*}: J^{r}(L)_{h, Y} \rightarrow J^{r}(L)_{h}$ is an epimorphism of right bialgebroids.

An entirely similar construction is possible if $J^{r}(L)_{h}$ is replaced with a LQFSAd $J^{\ell}(L)_{h}$.

Remark 4.2.4. Let $V^{\ell}(L)_{h} \in(\text { LQUEAd })_{A_{h}}$ be a quantization of $V^{\ell}(L)$. We have seen in $\S 4.2 .1$ that $V^{\ell}(L)_{h, Y}:=V^{\ell}(L)_{h} \widehat{\otimes}_{k} S(Z)$ is a LQUEAd which quantizes $V^{\ell}\left(L_{Q}\right)$. If $n \in \mathbb{N}$, let $S(Z)^{+}:=\operatorname{Ker}(\epsilon: S(Z) \longrightarrow k)$ be the kernel of the counit of $S(Z)$, and let $V^{\ell}(L)_{h, Y}^{* f, n}$ be the subspace of $V^{\ell}(L)_{h, Y}^{*}$ given by $V^{\ell}(L)_{h, Y}^{* f, n}:=$ $\left\{\lambda \in V^{\ell}(L)_{h, Y}^{*} \mid \lambda\left(V^{\ell}(L)_{h} \otimes_{k} S(Y)^{\otimes n} \otimes_{k} S(Z)^{+}\right)=0\right\}$. Then set

$$
V^{\ell}(L)_{h, Y}^{* f}:=h \text {-adic completion of } \sum_{n \in \mathbb{N}} V^{\ell}(L)_{h, Y}^{* f, n}
$$

Then $V^{\ell}(L)_{h, Y}^{*_{f}}$ is a right subbialgebroid of $V^{\ell}(L)_{h, Y}^{*}$, which is isomorphic to the right bialgebroid $\left(V^{\ell}(L)_{h}^{*}\right)_{Y}$. Note also that $V^{\ell}(L)_{h, Y}^{* f, n}$ is isomorphic to $V^{\ell}(L)_{h}^{*} \widetilde{\otimes}_{k} S\left(Y^{*}\right)^{\widetilde{\otimes}} \otimes_{k} 1 \otimes_{k} 1 \otimes_{k} 1 \cdots$

In a similar way, one can define also the right bialgebroid $\left(V^{\ell}(L)_{h, Y}\right)_{*_{f}}$ : this is a right subbialgebroid of $\left(V^{\ell}(L)_{h, Y}\right)_{*}$ isomorphic to the right bialgebroid $\left(\left(V^{\ell}(L)_{h}\right)_{*}\right)_{Y}$.

Parallel "right-handed versions" of the previous constructions and results also make sense if one starts with some $V^{r}(L)_{h} \in\left(\right.$ RQUEAd) $A_{h}$ instead of $V^{\ell}(L)_{h} \in$ (LQUEAd ${ }_{A_{h}}$ : in a nutshell, one still finds that "extension commutes with dualization". Details are left to the reader.

\section{Linear duality for quantum groupoids}

In this section we explore the relationship among quantum groupoids ruled by linear duality (i.e., by taking left or right duals). We shall see that the "(left/right) full 
dual" and the "(left/right) continuous dual" altogether provide category antiequivalences between (LQUEAd) $A_{h}$ and (RQFSAd) $A_{h}$ and between (RQUEAd) ${ }_{A_{h}}$ and (LQFSAd) $A_{h}$.

Essentially, we implement the construction of "dual bialgebroids" presented in Subsection 3.4, but still we need to make sure that several technical aspects do turn round.

5.1. Linear duality for QUEAd's. We begin with the construction of duals for (L/R)QUEAD's. In this case, we consider "full duals" (versus topological ones, cf. Subsection 5.2 later on. Before giving the main result, we need a couple of auxiliary, technical lemmas.

Lemma 5.1.1. Let $V^{\ell}(L)_{h} \in(\mathrm{LQUEAd})_{A_{h}}$ and $u \in V^{\ell}(L)_{h}$. For any $r \in \mathbb{N}$, there exists $t_{r} \in \mathbb{N}$ such that $\Delta^{t_{r}}(u)=\delta_{0}+h \delta_{1}+h^{2} \delta_{2}+\cdots+h^{r-1} \delta_{r-1}+h^{r} \delta_{r}(\epsilon$ $\left.V^{\ell}(L)_{h}^{\widehat{\otimes} t_{r}}\right)$ and, for any $i=0, \ldots, r-1$, each homogeneous tensor in an expansion of $\delta_{i}$ has at least $r$ factors equal to 1.

Proof. For any $w \in V^{\ell}(L)_{h}^{\otimes s}$, we denote by $\bar{w}$ the coset of $w$ modulo $h V^{\ell}(L)_{h}^{\otimes s}$.

We expand the given $u$ as $u=u_{0}+h u_{1}+\cdots+h^{r} u_{r}+\cdots$. Then there exists $t_{0}^{\prime} \in \mathbb{N}$ such that (each homogeneous tensor in) $\overline{\Delta^{t_{0}^{\prime}\left(u_{0}\right)}}$ contains at least $r$ terms equal to 1 . We lift $\overline{\Delta^{t_{0}^{\prime}}\left(u_{0}\right)}$ to some $\delta_{0}^{0} \in V^{\ell}(L)_{h}^{\widehat{\otimes} t_{0}^{\prime}}$ containing (i.e., its homogeneous tensors contain) at least $r$ terms equal to 1 . Then $\Delta^{t_{0}^{\prime}}(u)=\delta_{0}^{0}+h \delta_{1}^{0}+h^{2} \delta_{2}^{0}+\cdots+$ $h^{r} \delta_{r}^{0}+\cdots$ for suitable elements $\delta_{1}^{0}, \ldots, \delta_{r}^{0} \in V^{\ell}(L)_{h}^{\widehat{\otimes} t_{0}^{\prime}}$.

Now we can find $t_{1}^{\prime} \in \mathbb{N}$ such that $\overline{\left(i d^{t_{0}^{\prime}-1} \otimes \Delta^{t_{1}^{\prime}}\right)\left(\delta_{1}^{0}\right)}$ contains at least $r$ terms equal to 1 . We lift $\overline{\left(i d^{t_{0}^{\prime}-1} \otimes \Delta^{t_{1}^{\prime}}\right)\left(\delta_{0}^{0}\right)}$ and $\overline{\left(i d^{t_{0}^{\prime}-1} \otimes \Delta^{t_{1}^{\prime}}\right)\left(\delta_{1}^{0}\right)}$ to elements $\delta_{0}^{1}, \delta_{1}^{1} \in V^{\ell}(L)_{h}^{\widehat{\otimes} t_{0}^{\prime}+t_{1}^{\prime}}$ which both contain at least $r$ terms equal to 1 . Thus we find $\Delta^{t_{0}^{\prime}+t_{1}^{\prime}}(u)=\delta_{0}^{1}+h \delta_{1}^{1}+h^{2} \delta_{2}^{1}+\cdots+h^{r} \delta_{r}^{1}+\cdots$ for suitable $\delta_{2}^{1}, \ldots, \delta_{r}^{1} \in$ $V^{\ell}(L)_{h}^{\widehat{\otimes} t_{0}^{\prime}+t_{1}^{\prime}}$. Iterating finitely many times, we complete the proof.

Notation 5.1.2. Before the next lemma, we need some more notation: given $V^{\ell}(L)_{h} \in(\text { LQUEAd })_{h}$, consider $K_{h}:=V^{\ell}(L)_{h}^{*}$ and its subset $I_{K_{h}}:=\{\chi \in$ $\left.K_{h} \mid\left\langle 1_{V^{\ell}(L)_{h}}, \chi\right\rangle \in h A_{h}\right\}$.

Remark 5.1.3. As $V^{\ell}(L)_{h}$ is a left bialgebroid, by $\S 3.4 .2$ we know that its right dual $K_{h}:=V^{\ell}(L)_{h}^{*}$ has a canonical structure of $A^{e}$-ring; then, with respect to this structure, one easily sees that $I_{K_{h}}$ is a two-sided ideal of $K_{h}$. Moreover $\Delta\left(I_{h}\right) \subset$ $I_{K_{h}} \otimes K_{h}+K_{h} \otimes I_{K_{h}}$. Indeed, given any $\phi \in I_{K_{h}}$, we write $\Delta(\phi)=\phi_{(1)} \otimes \phi_{(2)}$ - a formal series (in $\Sigma$-notation) - convergent in the $I_{K_{h}} \widetilde{\otimes} K_{h}$-adic topology of 
$K_{h} \widetilde{\otimes} K_{h}$. Writing $\phi_{(1)}$ and $\phi_{(2)}$ as

$$
\begin{array}{llll}
\phi_{(1)}=\phi_{(1)}^{+s}+s^{r}\left(\partial_{h}\left(\phi_{(1)}\right)\right), & \text { with } & \phi_{(1)}^{+s}:=\phi_{(1)}-s^{r}\left(\partial_{h}\left(\phi_{(1)}\right)\right) \in I_{K_{h}} \\
\phi_{(2)}=\phi_{(2)}^{+t}+t^{r}\left(\partial_{h}\left(\phi_{(2)}\right)\right), & \text { with } & \phi_{(2)}^{+t}:=\phi_{(2)}-t^{r}\left(\partial_{h}\left(\phi_{(2)}\right)\right) \in I_{K_{h}}
\end{array}
$$

we can expand $\Delta(\phi)=\phi_{(1)} \otimes \phi_{(2)}$ as

$$
\begin{aligned}
\Delta(\phi)=\phi_{(1)}^{+s} \otimes \phi_{(2)}+s^{r}\left(\partial_{h}\left(\phi_{(1)}\right)\right) \otimes \phi_{(2)}^{+t}+s^{r}\left(\partial_{h}(\phi)\right) \otimes 1 \\
\quad \\
\quad\left(I_{K_{h}} \widetilde{\otimes}_{A_{h}} K_{h}+K_{h} \widetilde{\otimes}_{A_{h}} I_{K_{h}}+h s^{r}\left(A_{h}\right) \widetilde{\otimes}_{A_{h}} 1\right)
\end{aligned}
$$

where we took into account the identity $s^{r}\left(\partial_{h}\left(\phi_{(1)}\right)\right) \otimes t^{r}\left(\partial_{h}\left(\phi_{(2)}\right)\right)=s^{r}\left(\partial_{h}(\phi)\right) \otimes 1$, due to Remarks 3.3.2, and the fact that $\partial_{h}(\phi) \in h A_{h}$, since $\phi \in I_{K_{h}}$ by assumption.

Lemma 5.1.4. Given $V^{\ell}(L)_{h} \in(\operatorname{LQUEAd})_{A_{h}}$ and $K_{h}:=V^{\ell}(L)_{h}^{*}$, consider the two-sided ideal $I_{K_{h}}:=\left\{\chi \in K_{h} \mid\left\langle 1_{V^{\ell}(L)_{h}}, \chi\right\rangle \in h A_{h}\right\}$ of $K_{h}$, as well as its powers $I_{K_{h}}^{n}(n \in \mathbb{N})$. Then, for every $u \in V^{\ell}(L)_{h}$ and every $r \in \mathbb{N}$, there exists $t_{r} \in \mathbb{N}$ such that $\left\langle u, I_{K_{h}}^{t_{r}}\right\rangle \in h^{r} A_{h}$.

The same property holds if one considers the left dual $K_{h}:=\left(V^{\ell}(L)_{h}\right)_{*}$ of $V^{\ell}(L)_{h}$.

Proof. Thanks to the previous lemma, there exists $t_{r} \in \mathbb{N}$ such that

$$
\Delta^{t_{r}}(u)=\delta_{0}+h \delta_{1}+h^{2} \delta_{2}+\cdots+h^{r-1} \delta_{r-1}+h^{r} \delta_{r}
$$

for some elements $\delta_{0}, \ldots, \delta_{r} \in V^{\ell}(L)_{h}^{\widehat{\otimes} t_{r}}$ such that $\delta_{0}, \ldots, \delta_{r-1}$ contain at least $r$ terms equal to 1 . From this fact and the properties of the natural pairing $\langle$,$| between$ $V^{\ell}(L)_{h}$ and its right dual $K_{h}:=V^{\ell}(L)_{h}^{*}$ it is easy to see that $\langle\phi, u\rangle \in h^{r} A_{h}$ for all $\phi \in I_{K_{h}}^{t_{r}}$, whence the claim.

We are now ready for our first important result about linear duality of "quantum groupoids". In a nutshell, it claims that the left and the right dual of a left, resp. right, quantum universal enveloping algebroid are both right, resp. left, quantum formal series algebroids.

Theorem 5.1.5. (a) If $V^{\ell}(L)_{h} \in(\text { LQUEAd })_{A_{h}}$, then $V^{\ell}(L)_{h}^{*}, V^{\ell}(L)_{h *} \in(\operatorname{RQFSAd})_{A_{h}}$, with semiclassical limits ( $c f$. \$3.5.5)

$$
\begin{aligned}
V^{\ell}(L)_{h}^{*} / h V^{\ell}(L)_{h}^{*} & \cong V^{\ell}(L)^{*}=: J^{r}(L) \\
\text { and } \quad V^{\ell}(L)_{h *} / h V^{\ell}(L)_{h *} & \cong V^{\ell}(L)_{*} \cong J^{r}(L) .
\end{aligned}
$$

Therefore $V^{\ell}(L)_{h}^{*}$ and $V^{\ell}(L)_{h *}$ are quantizations of $J^{r}(L)$. 
Moreover, the structure of Lie-Rinehart algebra induced on $L^{*}$ by the quantization $V^{\ell}(L)_{h}^{*}$ of $J^{r}(L)$ - according to Theorem 4.1.12 - is the same as that induced by the quantization $V^{\ell}(L)_{h}$ of $V^{\ell}(L)$ - according to Theorem 4.1.3; therefore, the structure of Lie-Rinehart bialgebra induced on $L$ is the same in either case.

On the other hand, the structure of Lie-Rinehart algebra induced on $L^{*}$ by the quantization $V^{\ell}(L)_{h *}$ of $V^{\ell}(L)_{*} \cong J^{r}(L)$ is opposite to that induced by the quantization $V^{\ell}(L)_{h}$ of $V^{\ell}(L)$. Thus the structures of Lie-Rinehart bialgebra induced on $L$ in the two cases are coopposite to each other: $V^{\ell}(L)_{h}$ provides a quantization of the Lie-Rinehart bialgebra $L$, while $V^{\ell}(L)_{h *}$ provides a quantization of the coopposite Lie-Rinehart bialgebra $L_{\text {coop }}-c f$. Remarks 2.2.2 (e).

(b) If $V^{r}(L)_{h} \in(\text { RQUEAd })_{A_{h}}$, then ${ }_{*} V^{r}(L)_{h},{ }^{*} V^{r}(L)_{h} \in(\text { LQFSAd })_{A_{h}}$, with semiclassical limits (cf. §3.5.5)

$$
\begin{aligned}
&{ }^{*} V^{r}(L)_{h} / h_{*} V^{r}(L)_{h} \cong * V^{r}(L):=J^{\ell}(L) \\
& \text { and } \quad{ }^{*} V^{r}(L)_{h} / h^{*} V^{r}(L)_{h} \cong{ }^{*} V^{r}(L) \cong J^{\ell}(L)
\end{aligned}
$$

Therefore ${ }_{*} V^{r}(L)_{h}$ and ${ }^{*} V^{r}(L)_{h}$ are quantizations of $J^{\ell}(L):={ }_{*} V^{r}(L)$.

Moreover, the structures of Lie-Rinehart algebra induced on $L^{*}$ by the quantization ${ }_{*} V^{r}(L)_{h}$ of $J^{\ell}(L)$ - according to Theorem 4.1 .13 - is the same as that induced by the quantization $V^{r}(L)_{h}$ of $V^{r}(L)$-according to Theorem 4.1.6.

On the other hand, the structure of a Lie-Rinehart algebra induced on $L^{*}$ by the quantization ${ }^{*} V^{r}(L)_{h}$ of ${ }^{*} V^{r}(L) \cong J^{\ell}(L)$ is opposite to that induced by the quantization $V^{r}(L)_{h}$ of $V^{r}(L)$. Thus the structures of Lie-Rinehart bialgebra induced on $L$ in the two cases are coopposite to each other: $V^{\ell}(L)_{h}$ provides a quantization of the Lie-Rinehart bialgebra L, while ${ }^{*} V^{r}(L)_{h}$ provides a quantization of the coopposite Lie-Rinehart bialgebra $L_{\text {coop }}-c f$. Remarks 2.2.2 (e).

Proof. (a) We shall start by proving that if $V^{\ell}(L)_{h} \in\left(\right.$ LQUEAd) $A_{h}$, then $V^{\ell}(L)_{h}^{*} \in(\text { RQFSAd })_{A_{h}}$.

As we saw in $\$ 5.1 .2$, the right dual $K_{h}:=V^{\ell}(L)_{h}^{*}$ of $V^{\ell}(L)_{h}$ has a canonical structure of $A^{e}$-ring. Moreover, it is endowed with a map $\partial_{h}: V^{\ell}(L)_{h}^{*} \longrightarrow A_{h}(\chi \mapsto$ $\left.\left\langle 1_{V}^{\ell}(L)_{h}, \chi\right\rangle\right)$, which has all the properties of a "counit" in a right bialgebroid and defines the two-sided ideal $I_{K_{h}}:=\partial_{h}^{-1}\left(h A_{h}\right)$. What we still have to prove is that

- $K_{h}:=V^{\ell}(L)_{h}^{*}$ is complete for the $I_{K_{h}}$-adic topology;

- there exists a suitable coproduct $\Delta_{h}: K_{h}:=V^{\ell}(L)_{h}^{*} \rightarrow K_{h} \widetilde{\otimes}_{A_{h}} K_{h}=$ $V^{\ell}(L)_{h}^{*} \widetilde{\otimes}_{A_{h}} V^{\ell}(L)_{h}^{*}$, which makes $K_{h}:=V^{\ell}(L)_{h}^{*}$ into a topological right bialgebroid;

- $K_{h} / h K_{h}=V^{\ell}(L)_{h}^{*} / h V^{\ell}(L)_{h}^{*}$ is isomorphic to $V^{\ell}(L)^{*}$ as topological right bialgebroid. 
We begin by looking for an isomorphism $V^{\ell}(L)_{h}^{*} / h V^{\ell}(L)_{h}^{*} \cong V^{\ell}(L)^{*}$. For this, we distinguish two cases, the free one and the general one.

Free case: $L$ is a free A-module of finite type.

In this case, let us fix an $A$-basis $\left\{\bar{e}_{1}, \ldots, \bar{e}_{n}\right\}$ of the $A$-module $L$; then we lift each $\bar{e}_{i}$ to some $e_{i} \in V^{\ell}(L)_{h}$. Then any element of $V^{\ell}(L)_{h}$ can be written as the $h$-adic limit of elements of the form $\sum_{\left(a_{1}, \ldots, a_{n}\right) \in \mathbb{N}^{n}} t_{l}\left(c_{a_{1}, \ldots, a_{n}}\right) e_{1}^{a_{1}} \cdots e_{n}^{a_{n}}$ in which almost all $c_{a_{1}, \ldots, a_{n}}$ 's are zero.

For a given $\lambda \in V^{\ell}(L)^{*}$, set $\bar{\alpha}_{a_{1}, \ldots, a_{n}}:=\lambda\left(\bar{e}_{1}^{a_{1}} \ldots \bar{e}_{n}^{a_{n}}\right) \in A$ for all $\underline{a}:=$ $\left(a_{1}, \ldots, a_{n}\right) \in \mathbb{N}^{n}$. We lift each $\bar{\alpha}_{a_{1}, \ldots, a_{n}}$ to some $\alpha_{a_{1}, \ldots, a_{n}} \in A_{h}$, with the assumption that if $\bar{\alpha}_{a_{1}, \ldots, a_{n}}=0$ then we choose $\alpha_{a_{1}, \ldots, a_{n}}=0$. Now we set

$$
\Lambda\left(\sum_{\left(a_{1}, \ldots, a_{n}\right) \in \mathbb{N}^{n}} t_{l}\left(c_{a_{1}, \ldots, a_{n}}\right) e_{1}^{a_{1}} \cdots e_{n}^{a_{n}}\right):=\sum_{\left(a_{1}, \ldots, a_{n}\right) \in \mathbb{N}^{n}} c_{a_{1}, \ldots, a_{n}} \alpha_{a_{1}, \ldots, a_{n}}
$$

This defines a map $\Lambda$ from the right $A_{h}$-submodule of $V^{\ell}(L)_{h}$ spanned by all the monomials $e_{1}^{a_{1}} \cdots e_{n}^{a_{n}}$ to $A_{h}$ : as the $h$-adic completion of this submodule is nothing but $V^{\ell}(L)_{h}$, this map uniquely extends (by continuity) to a map $\Lambda$ : $V^{\ell}(L)_{h} \longrightarrow A_{h}$. By construction, we have $\Lambda \in V^{\ell}(L)_{h}^{*}$, and $\Lambda$ is a lifting of $\lambda$, that is $\Lambda \bmod h V^{\ell}(L)_{h}^{*}=\lambda$. This guarantees that the canonical map $V^{\ell}(L)_{h}^{*} / h V^{\ell}(L)_{h}^{*} \longrightarrow V^{\ell}(L)^{*}$, which is obviously injective, is also surjective.

General case: $L$ is a projective A-module of finite type.

As in $\$ 2.1 .6$, we introduce a projective $A$-module $Q$ such that $L \oplus Q=F$ is a finite free $A$-module. Fix an $A$-basis $\left\{b_{1}, \ldots, b_{n}\right\}$ of $F$, and set $Y=k b_{1} \oplus$ $k b_{2} \cdots \oplus k b_{n}$, so that $F=A \otimes_{k} Y$. The basis $\left\{b_{1}, \ldots, b_{n}\right\}$ also defines a good basis $\left\{\bar{e}_{i}\right\}_{i \in T:=\mathbb{N} \times\{1, \ldots, n\}}$ of $L_{Q}$.

Now let $\lambda \in V(L)^{*}$. We extend $\lambda$ to some $\lambda^{\prime} \in V\left(L_{Q}\right)^{*}$ by setting $\left.\lambda^{\prime}\right|_{V(L) \otimes S(Z)^{+}}:=0$. Now we can adapt the arguments of the free case to construct a lifting $\Lambda^{\prime} \in V(L)_{h, Y}{ }^{*}$ of $\lambda^{\prime}$. Then $\Lambda:=\left.\Lambda^{\prime}\right|_{V(L)_{h}} \in V(L)_{h}^{*}$ is a lifting of $\lambda$ as required.

Thus one sees again that the canonical map $V^{\ell}(L)_{h}^{*} / h V^{\ell}(L)_{h}^{*} \longrightarrow V^{\ell}(L)^{*}$ is a bijection.

On $V^{\ell}(L)_{h}^{*}$ we have already considered an algebraic structure of " $A^{e}$-ring with counit": the same structure then is inherited by its quotient $V^{\ell}(L)_{h}^{*} / h V^{\ell}(L)_{h}^{*}$. On the other hand, $V^{\ell}(L)^{*}$ is a right bialgebroid, hence in particular it is an " $A^{e}$-ring with counit" as well. The canonical bijection $V^{\ell}(L)_{h}^{*} / h V^{\ell}(L)_{h}^{*} \longrightarrow V^{\ell}(L)^{*}$ above is clearly compatible with this additional structure. In particular, this implies that $\operatorname{Ker}\left(\partial_{h}\right) \bmod h K_{h} \cong \operatorname{Ker}\left(\partial_{V^{\ell}(L)}\right)=: \mathfrak{J}_{V^{\ell}(L)^{*}}$.

Now consider $I_{K_{h}}:=\partial_{h}^{-1}\left(h A_{h}\right)$, which can be written as $I_{K_{h}}=\operatorname{Ker}\left(\partial_{h}\right)+$ $h K_{h}$. As we know that $V^{\ell}(L)^{*}$ is $\mathfrak{J}_{V^{\ell}(L)^{*-a d i c a l l y} \text { complete (cf. §3.5.1), from }}$ $\operatorname{Ker}\left(\partial_{h}\right) \bmod h K_{h} \cong \mathfrak{J}_{V^{\ell}(L)^{*}}$ and $I_{K_{h}}=\operatorname{Ker}\left(\partial_{h}\right)+h K_{h}$ we can easily argue that $K_{h}:=V^{\ell}(L)_{h}^{*}$ is $I_{K_{h}}$-adically complete. 
Now we look for a suitable coproduct. To this end, we shall show that the natural "coproduct" given by the recipe in $\$ 3.4 .2$ does the job. The problem is to prove the existence of an isomorphism from $V^{\ell}(L)_{h}^{*} \widetilde{\otimes} V^{\ell}(L)_{h}^{*}-$ the completion of $V^{\ell}(L)_{h}^{*} \otimes V^{\ell}(L)_{h}^{*}$ with respect to the topology defined by the filtration $\left(\sum_{p+q=n} I_{h}^{p} \otimes I_{h}^{q}\right)_{n \in \mathbb{N}}$ - to $\operatorname{Hom}_{\left(-, A_{h}\right)}\left(\left(V^{\ell}(L)_{h \triangleleft} \otimes V^{\ell}(L)_{h}\right)_{\triangleleft}, A_{h}\right)$. More precisely, there exists (cf. §3.4.2) a natural map $\chi$ from $V^{\ell}(L)_{h}^{*} \otimes V^{\ell}(L)_{h}^{*}$ to $\operatorname{Hom}_{\left(-, A_{h}\right)}\left(\left(V^{\ell}(L)_{h \triangleleft} \otimes_{\triangleleft} V^{\ell}(L)_{h}\right)_{\triangleleft}, A_{h}\right)$; we now show that this $\chi$ actually extends to a (continuous) map - which we still denote by $\chi-$ from $V^{\ell}(L)_{h}^{*} \triangleleft \widetilde{\otimes} \checkmark V^{\ell}(L)_{h}^{*}$ to $\operatorname{Hom}_{\left(-, A_{h}\right)}\left(\left(V^{\ell}(L)_{h \triangleleft} \otimes V^{\ell}(L)_{h}\right)_{\triangleleft}, A_{h}\right)$.

To begin with, fix $u \in V^{\ell}(L)_{h}$. For every $r \in \mathbb{N}$, there exists $t_{r} \in \mathbb{N}$ such that $\Delta^{t_{r}}(u)$ expands as $\Delta^{t_{r}}(u)=\delta_{0}+h \delta_{1}+h^{2} \delta_{2}+\cdots+h^{r} \delta_{r}$ as in Lemma 5.1.1: in particular, every $\delta_{i} \in V^{\ell}(L)_{h}^{\otimes t_{r}}$ with $0 \leq i \leq r-1$ contains at least $r$ terms equal to 1 . As the canonical evaluation pairing between $V^{\ell}(L)_{h}$ and $K_{h}:=V^{\ell}(L)_{h}^{*}$ is a bialgebroid right pairing — in the sense of Definition 3.4.5 - the formulas for such pairings imply at once (by induction) that $\left\langle u, I_{K_{h}}^{t}\right\rangle \subseteq h^{r} A_{h}$ for all $t \geq t_{r}$. By the same arguments, given $v, w \in V^{\ell}(L)_{h}$ we see that, for every $r \in \mathbb{N}$, one has

$$
\left\langle v \otimes w, I_{K_{h}}^{t^{\prime}} \otimes I_{K_{h}}^{t^{\prime \prime}}\right\rangle \subseteq h^{r} A_{h} \quad \text { for all } t^{\prime}+t^{\prime \prime} \gg 0
$$

Now let $\Lambda \in V^{\ell}(L)_{h}^{*} \widetilde{\otimes} \checkmark V^{\ell}(L)_{h}^{*}$. Then $\Lambda$ is the limit of a sequence $\left(\Lambda_{n}\right)_{n \in \mathbb{N}}$ — with $\Lambda_{n} \in V^{\ell}(L)_{h}^{*} \otimes_{\triangleright} V^{\ell}(L)_{h}^{*}$ for all $n$ - for the topology defined by the filtration $\left(\sum_{p+q=n} I_{h}^{p} \otimes I_{h}^{q}\right)_{n \in \mathbb{N}} ;$ in particular, for each $t \in \mathbb{N}$ one has

$$
\left(\Lambda_{n^{\prime}}-\Lambda_{n^{\prime \prime}}\right) \in \sum_{t^{\prime}+t^{\prime \prime}=t} I_{K_{h}}^{t^{\prime}} \otimes I_{K_{h}}^{t^{\prime \prime}} \quad \text { for all } n^{\prime}, n^{\prime \prime} \gg 0
$$

By (5.1) and (5.2) together we get that for all $r \in \mathbb{N}$ one has

$\left(\chi\left(\Lambda_{n^{\prime}}\right)-\chi\left(\Lambda_{n^{\prime \prime}}\right)\right)(v \otimes w)=\chi\left(\Lambda_{n^{\prime}}-\Lambda_{n^{\prime \prime}}\right)(v \otimes w):=\left\langle v \otimes w, \Lambda_{n^{\prime}}-\Lambda_{n^{\prime \prime}}\right\rangle \subseteq h^{r} A_{h}$

for all $n^{\prime}, n^{\prime \prime} \gg 0$; in other words, $\left\{\chi\left(\Lambda_{n}\right)(v \otimes w)\right\}_{n \in \mathbb{N}}$ is a Cauchy sequence for the $h$-adic topology in $A_{h}$; as the latter is $h$-adically complete (and separated), there exists a unique, well-defined limit $\lim _{n \rightarrow \infty} \chi\left(\Lambda_{n}\right)(v \otimes w) \in A_{h}$. In the end, we can set $\chi(\Lambda)(v \otimes w):=\lim _{n \rightarrow \infty} \chi\left(\Lambda_{n}\right)(v \otimes w)$; this defines a (continuous) map extending the original one, namely

$$
\chi: V^{\ell}(L)_{h \triangleleft}^{*} \widetilde{\otimes}_{\downarrow} V^{\ell}(L)_{h}^{*} \longrightarrow \operatorname{Hom}_{\left(-, A_{h}\right)}\left(\left(V^{\ell}(L)_{h \triangleleft} \otimes_{\triangleleft} V^{\ell}(L)_{h}\right)_{\triangleleft}, A_{h}\right)
$$

To complete our argument, we need a few more steps. In order to ease the notation, we shall write $\left.X\right|_{h=0}:=X / h X$ for every $k[[h]]$-module $X$. 
First, with the same arguments used to prove that $\left.\operatorname{Hom}_{\left(-, A_{h}\right)}\left(V^{\ell}(L)_{h}, A_{h}\right)\right|_{h=0}=$ $\left.V^{\ell}(L)_{h}^{*}\right|_{h=0}$ has a canonical bijection with $\operatorname{Hom}_{(-, A)}\left(V^{\ell}(L), A\right)=: V^{\ell}(L)^{*}$ we can also prove that

$$
\left.\operatorname{Hom}_{\left(-, A_{h}\right)}\left(\left(V^{\ell}(L)_{h \triangleleft} \otimes V^{\ell}(L)_{h}\right)_{\triangleleft}, A_{h}\right)\right|_{h=0} \cong \operatorname{Hom}_{(-, A)}\left(V^{\ell}(L) \otimes V^{\ell}(L), A\right)
$$

Similarly, the same arguments once more can be adapted to prove that

$$
\left.V^{\ell}(L)_{h \triangleleft}^{*} \widetilde{\otimes} \nabla V^{\ell}(L)_{h}^{*}\right|_{h=0} \cong V^{\ell}(L)^{*} \widetilde{\otimes} V^{\ell}(L)^{*} \quad\left(=J^{r}(L) \widetilde{\otimes} J^{r}(L),\right. \text { cf. §3.5.1) }
$$

Finally, by construction the reduction modulo $h$ of the map $\chi$ in (5.3), call it $\bar{\chi}$, is nothing but the map

$$
\widetilde{\vartheta}: J^{r}(L) \triangleleft \widetilde{\otimes} J^{r}(L)=V^{\ell}(L)_{\triangleleft}^{*} \widetilde{\otimes} V^{\ell}(L)^{*} \longrightarrow\left(V^{\ell}(L)_{\triangleleft} \otimes \nabla^{\ell}(L)\right)^{*}
$$

considered in \$3.5.1. Therefore - since $\operatorname{Hom}_{(-, A)}\left(V^{\ell}(L) \otimes V^{\ell}(L), A\right)=$ : $\left(V^{\ell}(L)_{\triangleleft} \otimes \nabla^{\ell}(L)\right)^{*}$, and taking into account the isomorphisms in (5.4-5) - as $\bar{\chi}=\widetilde{\vartheta}$ is a $k$-linear isomorphism we can deduce that $\chi$ is an isomorphism as well.

The outcome now is that $K_{h}:=V^{\ell}(L)_{h}^{*}$ endowed with the previously constructed structure - including the coproduct map given by the recipe in $\$ 3.4 .2$ is a topological right bialgebroid, complete with respect to the $I_{K_{h}}$-adic topology. In addition, the bijection $V^{\ell}(L)_{h}^{*} / h V^{\ell}(L)_{h}^{*} \longrightarrow V^{\ell}(L)^{*}$ found above by construction happens to be a right bialgebroid isomorphism.

Our next task is the following. Denote by $\left(L^{*},[,]^{\prime}, \omega^{\prime}\right)$ and $\left(L^{*},[,]^{\prime \prime}, \omega^{\prime \prime}\right)$ the structures of Lie-Rinehart bialgebras induced on $L^{*}$ respectively by Theorem 4.1.12 — for $J^{r}(L):=V^{\ell}(L)_{h}^{*}$ - and by Theorem 4.1.3 - applied to $V^{\ell}(L)_{h}$. We must prove that $\omega^{\prime}=\omega^{\prime \prime}$ and $[,]^{\prime}=[,]^{\prime \prime}$. To this end, recall that, by Remarks 2.2.2(b), $\omega^{\prime \prime}$ and $[,]^{\prime \prime}$ are uniquely determined by the conditions

$$
\begin{aligned}
\omega^{\prime \prime}(\Phi)(a) & =\left\langle\delta_{L}(a), \Phi\right\rangle, \\
\left\langle\Theta,[\Phi, \Psi]^{\prime \prime}\right\rangle & =\omega^{\prime \prime}(\Phi)(\langle\Theta, \Psi\rangle)-\omega^{\prime \prime}(\Psi)(\langle\Theta, \Phi\rangle)-\left\langle\delta_{L}(\Theta), \Phi \otimes \Psi\right\rangle
\end{aligned}
$$

(for all $\Phi, \Psi \in L^{*}, \Theta \in L, a \in A$ ), where $\delta_{L}(a)$ and $\delta_{L}(\Theta)$ are defined by the formula for $\delta$ in Theorem 4.1.3. Therefore, it is enough for us to prove that (for all $\Phi, \Psi \in L^{*}, \Theta \in L, a \in A$ )

$$
\begin{aligned}
\omega^{\prime}(\Phi)(a) & =\left\langle\delta_{L}(a), \Phi\right\rangle, \\
\left\langle\Theta,[\Phi, \Psi]^{\prime}\right\rangle & =\omega^{\prime \prime}(\Phi)(\langle\Theta, \Psi\rangle)-\omega^{\prime \prime}(\Psi)(\langle\Theta, \Phi\rangle)-\left\langle\delta_{L}(\Theta), \Phi \otimes \Psi\right\rangle
\end{aligned}
$$

In order to prove (5.6), we choose liftings $\phi^{\prime}, \psi^{\prime} \in J^{r}(L)_{h}:=V^{\ell}(L)_{h}^{*}$, with the additional condition that $\phi^{\prime}, \psi^{\prime} \in \mathfrak{J}_{J} r_{(L)_{h}}:=\operatorname{Ker}\left(\partial_{J} r(L)_{h}\right)$ (such a choice is 
always possible), a lifting $\theta \in V^{\ell}(L)_{h}$ of $\Theta$ and a lifting $a^{\prime} \in A_{h}$ of $a$. Now direct computation gives

$$
\begin{aligned}
\omega^{\prime} & (\Phi)(a)= \\
& =\left(\left(h^{-1}\left(\phi^{\prime} t_{r}\left(a^{\prime}\right)-t_{r}\left(a^{\prime}\right) \phi^{\prime}\right)\right) \bmod h J^{r}(L)_{h}\right) \bmod \mathfrak{J}_{J(L)} \\
& =\partial_{J(L)_{h}}\left(\frac{\phi^{\prime} t_{r}\left(a^{\prime}\right)-t_{r}\left(a^{\prime}\right) \phi^{\prime}}{h}\right) \bmod h A_{h} \\
& =\left\langle 1, \frac{\phi^{\prime} t_{r}\left(a^{\prime}\right)-t_{r}\left(a^{\prime}\right) \phi^{\prime}}{h}\right\rangle \bmod h A_{h}=\frac{a^{\prime}\left\langle 1, \phi^{\prime}\right\rangle-\left\langle 1, t_{r}\left(a^{\prime}\right) \phi^{\prime}\right\rangle}{h} \bmod h A_{h} \\
& =\frac{\left\langle 1, \phi^{\prime}\right\rangle a^{\prime}-\left\langle 1, t_{r}\left(a^{\prime}\right) \phi^{\prime}\right\rangle}{h} \bmod h A_{h}=\frac{\left\langle t^{\ell}\left(a^{\prime}\right), \phi^{\prime}\right\rangle-\left\langle s^{\ell}\left(a^{\prime}\right), \phi^{\prime}\right\rangle}{h} \bmod h A_{h} \\
& =\left\langle\frac{t^{\ell}\left(a^{\prime}\right)-s^{\ell}\left(a^{\prime}\right)}{h}, \phi^{\prime}\right\rangle \bmod h A_{h}=\left\langle\delta_{L}(a), \Phi\right\rangle
\end{aligned}
$$

where $\langle$,$\rangle denotes the natural evaluation pairing between V^{\ell}(L)_{h}$ and its right dual $V^{\ell}(L)_{h}^{*}$, we exploited the fact that this pairing is a right bialgebroid pairing (cf. Definitions 3.4.4 and 3.4.5) and the fact that $\left\langle 1_{V^{\ell}(L)_{h}}, \phi^{\prime}\right\rangle=: \partial_{J} r(L)_{h}\left(\phi^{\prime}\right)=0$ because $\phi^{\prime} \in \mathfrak{J}_{J^{r}(L)_{h}}:=\operatorname{Ker}\left(\partial_{J^{r}(L)_{h}}\right)$ by assumption. Thus the first identity in (5.6) is verified.

As to the second identity, we write $\Delta(\theta)=\theta_{(1)} \otimes \theta_{(2)}$ as $\Delta(\theta)=\theta \otimes 1+1 \otimes \theta+$ $h \sum_{[\theta]} \theta_{[1]} \otimes \theta_{[2]}$, so that $\left(\sum_{[\theta]} \theta_{[1]} \otimes \theta_{[2]}\right) \bmod h V^{\ell}(L)_{h \triangleleft} x_{A} V^{\ell}(L)_{h}=: \Delta^{[1]}(\Theta)$ — notation of Definition 4.1.3. Then by direct computation we find

$$
\begin{aligned}
& \left\langle\Theta,[\Phi, \Psi]^{\prime}\right\rangle= \\
& =\left\langle\theta, \frac{\phi^{\prime} \psi^{\prime}-\psi^{\prime} \phi^{\prime}}{h}\right\rangle \bmod h A_{h}=h^{-1}\left(\left\langle\theta, \phi^{\prime} \psi^{\prime}\right\rangle-\left\langle\theta, \psi^{\prime} \phi^{\prime}\right\rangle\right) \bmod h A_{h} \\
& =h^{-1}\left(\left\langle 1, t_{r}\left(\left\langle\theta, \phi^{\prime}\right\rangle\right) \psi^{\prime}\right\rangle-\left\langle 1, t_{r}\left(\left\langle\theta, \psi^{\prime}\right\rangle\right) \phi^{\prime}\right\rangle\right) \bmod h A_{h} \\
& \quad+\sum_{[\theta]}\left(\left\langle\theta_{[2]}, t_{r}\left(\left\langle\theta_{[1]}, \phi^{\prime}\right\rangle\right) \psi^{\prime}\right\rangle-\left\langle\theta_{[2]}, t_{r}\left(\left\langle\theta_{[1]}, \psi^{\prime}\right\rangle\right) \phi^{\prime}\right\rangle\right) \bmod h A_{h} \\
& =h^{-1}\left(\left\langle s^{\ell}\left(\left\langle\theta, \phi^{\prime}\right\rangle\right), \psi^{\prime}\right\rangle-\left\langle s^{\ell}\left(\left\langle\theta, \psi^{\prime}\right\rangle\right), \phi^{\prime}\right\rangle\right) \bmod h A_{h} \\
& \quad+\sum_{[\theta]}\left(\left\langle s^{\ell}\left(\left\langle\theta_{[1]}, \phi^{\prime}\right\rangle\right) \theta_{[2]}, \psi^{\prime}\right\rangle-\left\langle s^{\ell}\left(\left\langle\theta_{[1]}, \psi^{\prime}\right\rangle\right) \theta_{[2]}, \phi^{\prime}\right\rangle\right) \bmod h A_{h} \\
& =\left(h^{-1}\left\langle s^{\ell}\left(\left\langle\theta, \phi^{\prime}\right\rangle\right)-t^{\ell}\left(\left\langle\theta, \phi^{\prime}\right\rangle\right), \psi^{\prime}\right\rangle-h^{-1}\left\langle s^{\ell}\left(\left\langle\theta, \psi^{\prime}\right\rangle\right)-t^{\ell}\left(\left\langle\theta, \psi^{\prime}\right\rangle\right), \phi^{\prime}\right\rangle\right) \bmod h A_{h} \\
& \quad+\sum_{[\theta]}\left(\left\langle t^{\ell}\left(\left\langle\theta_{[1]}, \phi^{\prime}\right\rangle\right) \theta_{[2]}, \psi^{\prime}\right\rangle-\left\langle t^{\ell}\left(\left\langle\theta_{[1]}, \psi^{\prime}\right\rangle\right) \theta_{[2]}, \phi^{\prime}\right\rangle\right) \bmod h A_{h}
\end{aligned}
$$




$$
\begin{aligned}
= & \left(\left\langle\frac{s^{\ell}\left(\left\langle\theta, \phi^{\prime}\right\rangle\right)-t^{\ell}\left(\left\langle\theta, \phi^{\prime}\right\rangle\right)}{h}, \psi^{\prime}\right\rangle-\left\langle\frac{s^{\ell}\left(\left\langle\theta, \psi^{\prime}\right\rangle\right)-t^{\ell}\left(\left\langle\theta, \psi^{\prime}\right\rangle\right)}{h}, \phi^{\prime}\right\rangle\right) \bmod h A_{h} \\
& \quad+\sum_{[\theta]}\left(\left\langle\theta_{[2]}, \psi^{\prime}\right\rangle\left\langle\theta_{[1]}, \phi^{\prime}\right\rangle-\left\langle\theta_{[2]}, \phi^{\prime}\right\rangle\left\langle\theta_{[1]}, \psi^{\prime}\right\rangle\right) \bmod h A_{h} \\
=- & \left\langle\delta_{L}(\langle\Theta, \Phi\rangle), \Psi\right\rangle+\left\langle\delta_{L}(\langle\Theta, \Psi\rangle), \Phi\right\rangle+\left\langle\Delta^{[1]}(\Theta)-\Delta^{[1]}(\Theta)_{2,1}, \Phi \otimes \Psi\right\rangle \\
= & \omega^{\prime \prime}(\Phi)(|\Theta, \Psi\rangle)-\omega^{\prime \prime}(\Psi)(|\Theta, \Phi\rangle)-\left\langle\delta_{L}(\Theta), \Phi \otimes \Psi\right\rangle
\end{aligned}
$$

where we exploited the properties of a right bialgebroid pairing - in particular, the identity $\left\langle t^{\ell}(\alpha), \chi^{\prime}\right\rangle=\left\langle 1, \chi^{\prime}\right\rangle \alpha$ - the fact that $\left\langle 1, \phi^{\prime}\right\rangle=\partial_{J^{r}(L)_{h}}\left(\phi^{\prime}\right)=0,\left\langle 1, \psi^{\prime}\right\rangle=$ $\partial_{J} r_{(L)_{h}}\left(\psi^{\prime}\right)=0$, the fact that $s^{\ell}(\kappa) \equiv t^{\ell}(\kappa) \bmod h V^{\ell}(L)_{h}$ and the fact (already proved) that $\omega^{\prime \prime}=\omega^{\prime}$. This proves the second identity in (5.6).

Finally, we have to deal with $V^{\ell}(L)_{h *}$. Acting much like for $V^{\ell}(L)_{h}^{*}$, one proves that $V^{\ell}(L)_{h *}$ is indeed a topological right bialgebroid, whose specialization modulo $h$ is just $V^{\ell}(L)_{*} \cong J^{\ell}(L)$, hence we can claim that $V^{\ell}(L)_{h *} \in(\text { RQFSAd })_{A_{h}}$ is a quantization of $J^{\ell}(L)$.

As to the last part of claim $(a)$, concerning the two Lie-Rinehart algebra structures induced on $L^{*}$, we can again proceed like for $V^{\ell}(L)_{h}^{*}$ : the difference in the outcome - a minus sign — now is due to the fact that the natural pairing (given by evaluation) among the left bialgebroid $V^{\ell}(L)_{h}$ and the right bialgebroid $V^{\ell}(L)_{h *}$ is a left bialgebroid pairing (cf. Definitions 3.4.4 and 3.4.5) - whereas in the case of $V^{\ell}(L)_{h}$ and $V^{\ell}(L)_{h}^{*}$ it is a right bialgebroid pairing. Full detail computations are left to the reader.

(b) The proof given for claim (a) clearly adapts to claim (b) as well, by the same arguments. Otherwise, one can deduce claim (b) directly from claim (a) using general isomorphisms such as $*\left(U_{\text {coop }}^{o p}\right) \cong\left(U^{*}\right)_{\text {coop }}^{o p}$ and $*\left(U_{\text {coop }}^{o p}\right) \cong\left(U_{*}\right)_{\text {coop }}^{o p}$ (see Remark 3.4.6).

5.2. Linear duality for QFSAd's. Much like for their classical counterparts, the duals for QFSAD's have to be meant in topological sense. Indeed, we introduce now a suitable definition of "continuous" dual of a (R/L)QFSAd:

Definition 5.2.1. Let $K_{h} \in(\mathrm{RQFSAd})_{A_{h}}$. Let $I_{h}:=\left\{\lambda \in K_{h} \mid \partial_{h}(\lambda) \in h A_{h}\right\}$.

We denote by ${ }_{\star} K_{h}$ the $k[[h]]$-submodule of ${ }_{*} K_{h}$ of all (left $A_{h}$-linear) maps from $K_{h}$ to $A_{h}$ which are continuous for the $I_{h}$-adic topology on $K_{h}$ and the $h$-adic topology on $A_{h}$.

We denote by ${ }^{\star} K_{h}$ the $k[[h]]$-submodule of ${ }^{*} K_{h}$ of all (right $A_{h}$-linear) maps from $K_{h}$ to $A_{h}$ which are continuous for the $I_{h}$-adic topology on $K_{h}$ and the $h$-adic topology on $A_{h}$.

In a similar way, we define also "continuous dual" objects $K_{h \star}\left(\subseteq K_{h *}\right)$ and $K_{h}^{\star}\left(\subseteq K_{h}^{*}\right)$ for every $K_{h} \in(\mathrm{LQFSAd})_{A_{h}}$. 
It is time for our second result about linear duality of "quantum groupoids". In short, it claims that the left and the right continuous dual of a left, resp. right, quantum formal series algebroid are both right, resp. left, quantum universal enveloping algebroids.

Theorem 5.2.2. (a) If $J^{r}(L)_{h} \in(\mathrm{RQFSAd})_{A_{h}}$, then ${ }_{\star} J^{r}(L)_{h},{ }^{\star} J^{r}(L)_{h} \in(\text { LQUEAd })_{A_{h}}$, with semiclassical limits (cf. Remark 3.5.8)

$$
\begin{aligned}
{ }^{\star} J^{r}(L)_{h} / h_{\star} J^{r}(L)_{h} & \cong{ }_{\star} J^{r}(L)=V^{\ell}(L) \\
\text { and } \quad{ }^{\star} J^{r}(L)_{h} / h^{\star} J^{r}(L)_{h} & \cong{ }^{\star} J^{r}(L) \cong V^{\ell}(L)
\end{aligned}
$$

Therefore ${ }_{\star} J^{r}(L)_{h}$ and ${ }^{\star} J^{r}(L)_{h}$ are quantizations of $V^{\ell}(L)={ }_{\star} J^{r}(L)$.

Moreover, the structure of Lie-Rinehart bialgebra induced on $L$ by the quantization ${ }_{\star} J^{r}(L)_{h}$ of $V^{\ell}(L)$ - according to Theorem 4.1 .3 - is the same as that induced by the quantization $J^{r}(L)_{h}$ of $J^{r}(L)$ - according to Theorem 4.1.12.

On the other hand, the structure of Lie-Rinehart algebra induced on $L^{*}$ by the quantization ${ }^{\star} J^{r}(L)_{h}$ of $V^{\ell}(L)$ is opposite to that induced by the quantization $J^{r}(L)_{h}$ of $J^{r}(L)$. Therefore, the structures of Lie-Rinehart bialgebra induced on $L$ in the two cases are coopposite to each other: in short, $J^{r}(L)_{h}$ provides a quantization of the Lie-Rinehart bialgebra $L$, while ${ }^{\star} J^{r}(L)_{h}$ provides a quantization of the coopposite Lie-Rinehart bialgebra $L_{\text {coop }}-c f$. Remarks 2.2.2 (e).

(b) If $J^{\ell}(L)_{h} \in(\text { LQFSAd })_{A_{h}}$, then $J^{\ell}(L)_{h}^{\star}, J^{\ell}(L)_{h_{\star}} \in(\text { RQUEAd })_{A_{h}}$, with semiclassical limits (cf. Remark 3.5.8)

$$
\begin{aligned}
J^{\ell}(L)_{h}^{\star} / h J^{\ell}(L)_{h}^{\star} & \cong J^{\ell}(L)^{\star}=V^{r}(L) \\
\text { and } \quad J^{\ell}(L)_{h_{\star}} / h J^{\ell}(L)_{h_{\star}} & \cong J^{\ell}(L)_{\star} \cong V^{r}(L)
\end{aligned}
$$

Therefore $J^{\ell}(L)_{h}^{\star}$ and $J^{\ell}(L)_{h_{\star}}$ are quantizations of $V^{r}(L)=J^{\ell}(L)^{\star}$.

Moreover, the structure of Lie-Rinehart bialgebra induced on $L$ by the quantization $J^{\ell}(L)_{h}^{\star}$ of $V^{r}(L)$ - according to Theorem 4.1.6-is the same as that induced by the quantization $J^{\ell}(L)_{h}$ of $J^{\ell}(L)$ - according to Theorem 4.1.13.

On the other hand, the structure of Lie-Rinehart algebra induced on $L^{*}$ by the quantization $J^{\ell}(L)_{h_{\star}}$ of $V^{r}(L)$ is opposite to that induced by the quantization $J^{\ell}(L)_{h}$ of $J^{\ell}(L)$. Therefore, the structures of Lie-Rinehart bialgebra induced on $L$ in the two cases are coopposite to each other: in short, $J^{\ell}(L)_{h}$ provides a quantization of the Lie-Rinehart bialgebra L, while $J^{\ell}(L)_{h_{\star}}$ provides a quantization of the coopposite Lie-Rinehart bialgebra $L_{\text {coop }}-c f$. Remarks 2.2.2 (e).

Proof. (a) To simplify notation set $K_{h}:=J^{r}(L)_{h}$. We begin dealing with ${ }_{\star} K_{h}$, in several steps.

The main point in the proof is the following. By definition, ${ }_{\star} K_{h}$ is contained in ${ }_{*} K_{h}$ : by the recipe in $\$ 3.4$, the latter is "almost" a left bialgebroid over $A_{h}$, as it has a natural structure of $A_{h}^{e}$-ring with "counit", and also a "candidate" as coproduct. 
Then the natural pairing among ${ }_{*} K_{h}$ and $K_{h}$ (given by evaluation), hereafter denoted $\langle$,$\rangle , is an A_{h}^{e}$-right pairing (cf. Definition 3.4.4), and also a bialgebroid right pairing (cf. Definition 3.4.5) - as far as this makes sense. Basing on this, we shall presently show that this structure on ${ }_{*} K_{h}$ - which makes it an $A_{h}^{e}$-ring and even "almost a left $A_{h}^{e}$-bialgebroid", actually does restrict to ${ }_{\star} K_{h}$, making it into a left $A_{h}^{e}$-bialgebroid. Also, the evaluation will then provide a natural bialgebroid right pairing between ${ }_{\star} K_{h}$ and $K_{h}$.

Along the way, we shall prove also that ${ }_{\star} K_{h}$ has semiclassical limit $V^{\ell}(L)$, and finally that the Lie-Rinehart bialgebra structure on $L$ induced by it is the same as that induced by $K_{h}:=J^{r}(L)_{h}$.

(1) First we prove that the source and target maps of ${ }_{*} K_{h}$ (as given in §3.4) actually map into ${ }_{\star} K_{h}$, that is $S_{*}^{\ell}\left(A_{h}\right) \subseteq{ }_{\star} K_{h}$ and $t_{*}^{\ell}\left(A_{h}\right) \subseteq{ }_{\star} K_{h}$. We shall prove it by showing that, for any $a \in A_{h}$, one has $\left\langle s_{*}^{\ell}(a), I_{h}^{n}\right\rangle \subseteq h^{n} A_{h},\left\langle t_{*}^{\ell}(a), I_{h}^{n}\right\rangle \subseteq h^{n} A_{h}$, for all $n \in \mathbb{N}$. $h^{1} A_{h}$.

For $t_{*}^{\ell}$, if $\phi \in I_{h}$, then $\left\langle t_{*}^{\ell}(a), \phi\right\rangle=\langle 1, \phi| a \in h A_{h}=h^{1} A_{h}$; thus $\left\langle t_{*}^{\ell}(a), I_{h}^{1}\right\rangle \subseteq$

Now assume by induction that $\left\langle t_{*}^{\ell}(a), I_{h}^{m}\right\rangle \subseteq h^{m} A_{h}$. Let $\psi \in I_{h}^{m}$ and $\chi \in I_{h}$; then

$$
\left\langle t_{*}^{\ell}(a), \psi \chi\right\rangle=\langle 1, \psi \chi\rangle a=\left\langle s_{*}^{\ell}(\langle 1, \psi\rangle) 1, \chi\right\rangle a
$$

thus by the induction hypothesis and the case $m=1$ we see that $\left\langle t_{*}^{\ell}(a), \psi \chi\right\rangle \in$ $h^{m+1} A_{h}$.

The case of $s_{*}^{\ell}$ - being totally similar — is left to the reader.

(2) Let us show that if $\omega, \omega^{\prime} \in{ }_{\star} K_{h}$, then $\omega \omega^{\prime} \in{ }_{\star} K_{h}$. Given $n \in \mathbb{N}$, let $p, q \in \mathbb{N}$ be such that $\left\langle\omega, I_{h}^{p}\right\rangle \in h^{n} A_{h}$ and $\left\langle\omega^{\prime}, I_{h}^{q}\right\rangle \in h^{n} A_{h}$. Now take $\eta \in I_{h}^{p+q}$. Then the identity

$$
\left\langle\omega \omega^{\prime}, \eta\right\rangle=\left\langle\omega t_{*}^{\ell}\left(\left\langle\omega^{\prime}, \eta_{(2)}\right\rangle\right), \eta_{(1)}\right\rangle=\left\langle\omega, \eta_{(1)} s_{r}\left(\left\langle\omega^{\prime}, \eta_{(2)}\right\rangle\right)\right\rangle
$$

taking into account that $\Delta\left(I_{h}^{p+q}\right) \subseteq \sum_{r+s=p+q} I_{h}^{r} \otimes_{A_{h}} I_{h}^{s}$ because $\Delta\left(I_{h}\right) \subseteq K_{h} \otimes_{A_{h}}$ $I_{h}+I_{h} \otimes_{A_{h}} K_{h}$, proves that $\left\langle\omega \omega^{\prime}, I_{h}^{p+q}\right\rangle \in h^{n} A_{h}$. Thus ${ }_{\star} K_{h}$ is a subring of ${ }_{*} K_{h}-$ even an $A_{h}^{e}$-subring, by (1).

(3) Let us show that ${ }_{\star} K_{h}$ is topologically free. First we prove that it is complete for the $h$-adic topology. Indeed, as $K_{h}$ is topologically free (for its own $h$ adic topology), so is $\operatorname{Hom}_{k[[h]]}\left(K_{h}, A_{h}\right)$ as well. Now let $\left(\lambda_{n}\right)_{n \in \mathbb{N}}$ be a Cauchy sequence of elements in ${ }_{\star} K_{h}$; then this sequence converges to a unique limit $\lambda \in \operatorname{Hom}_{k[[h]]}\left(K_{h}, A_{h}\right)$. Then it is easy to see that $\lambda \in \operatorname{Hom}_{A_{h}}\left(K_{h}, A_{h}\right)$.

Now we show that $\lambda \in{ }_{\star} K_{h}$. Given $n \in \mathbb{N}$, there exists $n_{1} \in \mathbb{N}$ such that $\lambda_{n_{1}}-\lambda$ takes values in $h^{n} A_{h}$. As $\lambda_{n_{1}} \in{ }_{\star} K_{h}$, there exists $n_{2} \in \mathbb{N}$ such that $\left\langle\lambda_{n_{1}}, I_{h}^{n_{2}}\right\rangle \in h^{n} A_{h}$. But then we have $\left\langle\lambda, I_{h}^{n_{2}}\right\rangle \in h^{n} A_{h}$ and so we conclude that $\lambda \in{ }_{\star} K_{h}$. 
Finally, as ${ }_{\star} K_{h}$ is complete for the $h$-adic topology and without torsion, it is topologically free.

(4) Now we show that ${ }_{\star} K_{h} / h_{\star} K_{h}={ }_{\star}\left(K_{h} / h K_{h}\right)={ }_{\star} J^{r}(L)=V^{\ell}(L)$.

Let $\lambda \in{ }_{\star} K_{h}$, so that $\lambda$ as a map from $K_{h}$ (with the $I_{h}$-adic topology) to $A_{h}$ (with the $h$-adic topology) is continuous. Then $\lambda$ induces (modulo $h$ ) a map $\bar{\lambda}: J^{r}(L) \longrightarrow A$ which is 0 on $\mathfrak{J}^{n}$ for $n \gg 0$, where $\mathfrak{J}:=\operatorname{Ker}\left(\partial_{J^{r}}{ }^{(L)}\right)$. We claim that the kernel of the map $\chi: \lambda \mapsto \bar{\lambda}$ is $h_{\star} K_{h}$ : indeed, it is obvious that $h_{\star} K_{h} \subseteq \operatorname{Ker}(\chi)$, and the inverse inclusion follows from the fact that $A_{h}$ is topologically free. Therefore we have an injective map $\bar{\chi}:{ }_{\star} K_{h} / h_{\star} K_{h} \longrightarrow$ ${ }_{\star}\left(K_{h} / h K_{h}\right)={ }_{\star} J^{r}(L)=V^{\ell}(L)$ induced by $\chi(\operatorname{modulo} h)$, and we are left to show that $\bar{\chi}$ is surjective too.

We distinguish two cases:

Finite free case: Assume that $L$ as an $A$-module is free of finite type. Let $\left\{\bar{e}_{1}, \ldots, \bar{e}_{n}\right\}$ be an $A$-basis of $L$. Then $\left\{\underline{\underline{e}}^{\underline{\alpha}}:=\bar{e}_{1}^{\alpha_{1}} \ldots \bar{e}_{n}^{\alpha_{n}} \mid \underline{\alpha}:=\left(\alpha_{1}, \ldots, \alpha_{n}\right) \in \mathbb{N}^{n}\right\}$ is a basis of $V^{\ell}(L)$, by the Poincaré-Birkhoff-Witt theorem. Define $\bar{\xi}_{i} \in K:=$ $J^{r}(L)$ by $\left\langle\bar{\xi}_{i}, \bar{e}_{1}^{\alpha_{1}} \cdots \bar{e}_{n}^{\alpha_{n}}\right\rangle=\prod_{j=1}^{n} \delta_{\alpha_{j}, \delta_{i, j}}$.

Let $\xi_{i} \in K_{h}$ be a lifting of $\bar{\xi}_{i}$ such that $\partial_{h}\left(\xi_{i}\right)=0$. We denote (ordered) monomials in the $\bar{\xi}_{i}$ 's or in the $\xi_{i}$ 's by $\underline{\xi}^{\underline{\alpha}}:=\bar{\xi}_{1}^{\alpha_{1}} \cdots \bar{\xi}_{n}^{\alpha_{n}}$ and $\underline{\xi}^{\underline{\alpha}}:=\xi_{1}^{\alpha_{1}} \cdots \xi_{n}^{\alpha_{n}}$ respectively. Note that $\underline{\xi}^{\underline{\alpha}} \in I_{h}^{|\underline{\alpha}|}$, where $|\underline{\alpha}|:=\sum_{i=1}^{n} \alpha_{i}$. Let $\lambda \epsilon_{\star}\left(K_{h} / h K_{h}\right)=$ ${ }_{\star} J^{r}(L)=V^{\ell}(L)$ be given: we write $\bar{a}_{\underline{\alpha}}:=\left\langle\lambda, \underline{\xi^{\alpha}}\right\rangle \in A$, and note that all but finitely many of the $\bar{a}_{\underline{\alpha}}$ 's are zero. Let $a_{\underline{\alpha}} \in \bar{A}_{h}$ be any lifting of $\bar{a}_{\underline{\alpha}}$ (for all $\underline{\alpha} \in \mathbb{N}^{n}$ ), with the condition that whenever $\bar{a}_{\underline{\alpha}}=0$ we take also $a_{\underline{\alpha}}=0$. Now we define $\Lambda \in{ }_{*} K_{h}$ by setting $\left\langle\Lambda, \underline{\xi}^{\underline{\alpha}}\right\rangle:=a_{\underline{\alpha}}$. As $I_{h}^{m}=\sum_{|\underline{\alpha}|+s \geq m} h^{s} \underline{\underline{\xi}} t_{r}\left(A_{h}\right)$, it is easy to check that if $n \in \mathbb{N}$ then $\left\langle\Lambda, I_{h}^{m}\right\rangle \subseteq h^{n} A_{h}$ for $m \gg 0$. Hence $\Lambda \in{ }_{\star} K_{h}$, and by construction $\bar{\chi}(\Lambda$ $\left.\bmod h_{\star} K_{h}\right)=\lambda$, so that the map $\bar{\chi}$ is onto, q.e.d.

General case: By our overall assumption, $L$ as an $A$-module is projective of finite type. Then we resume the setup and notation of in \$2.1.6: there exists a finitely generated projective $A$-module $Q$ such that $L \oplus Q=F$ is a finite free $A$-module, and we consider the free $A$-module $L_{Q}:=L \oplus\left(A \otimes_{k} Z\right)$ with $Z:=Y \oplus Y \oplus Y \oplus \cdots$. From an $A$-basis $\left\{b_{1}, \ldots, b_{n}\right\}$ of $Y$ we get a "good basis" of elements $\bar{e}_{t}$ indexed by $T:=\mathbb{N} \times\{1, \ldots, n\}$, i.e. $L_{Q}=\underset{t \in T}{\oplus} k \bar{e}_{t}$. Fixing on $T$ any total order, the PBW theorem yields $\left\{\underline{\underline{e}}^{\underline{\alpha}}:=\prod_{t \in T} \bar{e}_{t}^{\alpha_{t}} \mid \underline{\underline{\alpha}}=\left(\alpha_{t}\right)_{t \in T} \in T^{(\mathbb{N})}\right\}$ is an $A$-basis of $V^{\ell}\left(L_{Q}\right)$. Let $\bar{\xi}_{j}$ be the element of $J_{f}^{r}\left(L_{Q}\right)$ defined by $\left\langle\bar{\xi}_{j}, \underline{\underline{e}}^{\underline{\alpha}}\right\rangle=1$ if $\underline{\alpha}=\left(\alpha_{t}=\delta_{t, j}\right)_{t \in T}$, $\left\langle\bar{\xi}_{j}, \underline{\underline{e}}^{\underline{\alpha}}\right\rangle=0$ otherwise. If $A\left[\left[\left\{X_{t}\right\}_{t \in T}\right]\right]_{f}:=\bigcup_{i_{1}<\cdots<i_{n}} A\left[\left[X_{i_{1}}, \ldots, X_{i_{n}}\right]\right]$, then one has $J_{f}^{r}\left(L_{Q}\right)=A\left[\left[\left\{\bar{\xi}_{t}\right\}_{t \in T}\right]\right]_{f}$.

Now consider the quantization $K_{h, Y}$ of $J_{f}^{r}\left(L_{Q}\right)-$ cf. §4.2.3. Recall (cf. §4.2.3) that

$$
K_{h, Y}:=h \text {-adic completion of } \sum_{n \in \mathbb{N}} K_{h} \widetilde{\otimes}_{k} S\left(Y^{*}\right)^{\widetilde{\otimes} n} \otimes 1 \otimes 1 \otimes 1 \cdots
$$


where $K_{h} \widetilde{\otimes}_{k} S\left(Y^{*}\right)^{\widetilde{\otimes}} \otimes 1 \otimes 1 \cdots$ is the $\left(\left(S\left(Y^{*}\right)^{\otimes n}\right)^{+} \otimes 1 \otimes 1 \cdots\right)$-adic completion of $K_{h} \otimes_{k} S\left(Y^{*}\right)^{\otimes n} \otimes 1 \otimes 1 \cdots$. By construction, every $\bar{\xi}_{i}$ belongs to some $K \widetilde{\otimes}_{k} S\left(Y^{*}\right)^{\widetilde{\otimes} n_{i}} \otimes_{k} 1 \otimes_{k} 1 \otimes_{k} \cdots\left(n_{i} \in \mathbb{N}\right)$. Let $\xi_{i}$ be any lifting of $\bar{\xi}_{i}$ in $K_{h} \widetilde{\otimes}_{k} S\left(Y^{*}\right)^{\widetilde{\otimes} n_{i}} \otimes_{k} 1 \otimes_{k} 1 \otimes_{k} \cdots$ such that $\left(\partial_{h} \otimes \epsilon_{S\left(Z^{*}\right)}\right)\left(\xi_{i}\right)=0$. Given $a \in A_{h}$, we denote again by $a$ the element $t^{r}(a) \in t^{r}\left(A_{h}\right) \subseteq K_{h}$. Let also $\sigma: A \hookrightarrow A_{h}$ be a section of the natural projection map from $A_{h}$ to $A$, let $\mathfrak{J}_{h, Y}:=\operatorname{Ker}\left(\partial_{h}\right)=\partial_{h}^{-1}(\{0\})$ and $I_{h, Y}:=\partial_{h}^{-1}\left(h A_{h}\right)$ : taking into account that $t^{r}(A)=A^{o p}$ and $t^{r}\left(A_{h}\right)=A_{h}^{o p}$, one has

$$
\begin{aligned}
K_{h, Y} & =\left\{\sum_{n \in \mathbb{N}} h^{n} P_{n}^{\sigma}(\underline{\xi}) \mid P_{n} \in\left[\left[\left\{X_{t}\right\}_{t \in T}\right]\right]_{f} A^{o p}\right\} \\
& =\left\{\sum_{n \in \mathbb{N}} h^{n} P_{n}(\underline{\xi}) \mid P_{n} \in\left[\left[\left\{X_{t}\right\}_{t \in T}\right]\right]_{f} A_{h}^{o p}\right\} \\
I_{h, Y} & =\left(h,\left\{\xi_{t}\right\}_{t \in T}\right), \\
\mathfrak{J}_{h, Y} & =\sum_{t \in T} \xi_{t} K_{h, Y}
\end{aligned}
$$

where round braces stand for "two-sided ideal generated by", and $\left[\left[\left\{X_{t}\right\}_{t \in T}\right]\right]_{f} A$, respectively $\left[\left[\left\{X_{t}\right\}_{t \in T}\right]\right]_{f} A_{h}$, denotes the ring of formal power series with coefficients on the right chosen in $A$, respectively in $A_{h}$, each one involving only finitely many indeterminates $X_{t}$.

Now, $L_{Q}$ as an $A$-module is free but not finite; however, $J_{f}^{r}\left(L_{Q}\right)$ and its quantization $K_{h, Y}$ have enough "finiteness" behavior as to let the arguments for the finite free case apply again. In other words, the analysis we carried on for the finite free case can be applied again in the present, general context working with $K_{h, Y}$. Indeed, let us remark that

$$
\begin{aligned}
& I_{h, Y}:=h \text {-adic completion of } \\
& \qquad\left(\sum_{n \in \mathbb{N}} I_{h} \underset{k}{\widetilde{\otimes}} S\left(Y^{*}\right)^{\widetilde{\otimes}_{k} n} \underset{k}{\otimes} \underset{k}{\otimes} \cdots+\sum_{n \in \mathbb{N}_{+}} K_{h} \underset{k}{\widetilde{\otimes}}\left(S\left(Y^{*}\right)^{\widetilde{\otimes}_{k} n}\right)^{+} \underset{k}{\otimes} \underset{k}{1} \cdots\right)
\end{aligned}
$$

while on the other hand $V^{\ell}\left(L_{Q}\right)=V^{\ell}(L) \oplus\left(V^{\ell}(L) \otimes_{k} S(Z)^{+}\right)$. Now let $K:=$ $J^{r}(L)$ and $\lambda \in{ }_{\star} K$. As $J_{f}^{r}\left(L_{Q}\right)=K \oplus \sum_{n \in \mathbb{N}_{+}} K \widetilde{\otimes}_{k}\left(S\left(Y^{*}\right)^{\widetilde{\otimes} n}\right)^{+} \otimes_{k} 1 \otimes_{k} \cdots$, we can extend $\lambda$ to an element $\mu \in{ }_{\star} J_{f}^{r}\left(L_{Q}\right)$ by $\left.\mu\right|_{\sum_{n \in \mathbb{N}_{+}} K \widetilde{\otimes}_{k}\left(S\left(Y^{*}\right)^{\otimes n}\right)^{+} \otimes_{k} 1 \otimes_{k} \ldots}:=0$ and $\left.\mu\right|_{K}:=\lambda$. By the arguments used in the finite free case, $\mu$ can be lifted to an element $M \in{ }_{\star_{f}} K_{h, Y}$; then $\Lambda=\left.M\right|_{K_{h}} \in{ }_{\star} K_{h}$ is a lift of $\lambda$. So the (injective) map $\bar{\chi}:{ }_{\star} K_{h} / h_{\star} K_{h} \longrightarrow{ }_{\star}\left(K_{h} / h K_{h}\right)={ }_{\star} J^{r}(L)=V^{\ell}(L)$ is surjective.

(5) Let us now show that $\Delta\left({ }_{\star} K_{h}\right) \subseteq{ }_{\star} K_{h} \widehat{\otimes}_{A_{h}{ }^{\star}} K_{h}$ for the "coproduct map" $\Delta$ given by the transpose map of the multiplication in $K_{h}$.

Let $\Lambda \in{ }_{\star} K_{h}$. We know that modulo $h$ one has $\overline{\Delta(\Lambda)} \in{ }_{\star} K \otimes_{A}{ }_{\star} K$. Now write $\overline{\Delta(\Lambda)}=\sum \lambda^{(1)} \otimes \lambda^{(2)}$ (a finite sum) with $\lambda^{(1)}, \lambda^{(2)} \in{ }_{\star} K$, and let $\Lambda_{h}^{(1)}$ 
and $\Lambda_{h}^{(2)}$ in ${ }_{\star} K_{h}$ be liftings of $\lambda^{(1)}$ and $\lambda^{(2)}$, i.e. $\overline{\Lambda_{h}^{(1)}}=\lambda^{(1)}$ and $\overline{\Lambda_{h}^{(2)}}=\lambda^{(2)}$ : then $\Delta\left(\Lambda_{h}\right)-\sum \Lambda_{h}^{(1)} \otimes \Lambda_{h}^{(2)} \in h\left({ }_{*} K_{h} \widehat{\otimes}_{A_{h}} K_{h}\right)$, so that $h^{-1}\left(\Delta\left(\Lambda_{h}\right)-\sum \Lambda_{h}^{(1)} \otimes \Lambda_{h}^{(2)}\right) \in$ ${ }_{*} K_{h} \widehat{\otimes}_{A_{h}}{ }^{*} K_{h}$. In addition, whenever $p+q \gg 0$ one has also $\left\langle\Delta\left(\Lambda_{h}\right)-\sum \Lambda_{h}^{(1)} \otimes\right.$ $\left.\Lambda_{h}^{(2)}, I_{h}^{p} \otimes I_{h}^{q}\right\rangle \in h^{2} A_{h}$; therefore we find that

$$
\overline{h^{-1}\left(\Delta\left(\Lambda_{h}\right)-\sum \Lambda_{h}^{(1)} \otimes \Lambda_{h}^{(2)}\right)} \in{ }_{\star} K \otimes_{A}{ }_{\star} K
$$

We can carry on this argument and eventually show that $\Delta\left(\Lambda_{h}\right) \in{ }_{\star} K_{h} \widehat{\otimes}_{A_{h}{ }^{\star}} K_{h}$, q.e.d.

(6) Altogether, the steps (1)-(5) above prove that ${ }_{\star} K_{h}$ is a LQUEAd (over $A_{h}$ ), whose semiclassical limit ${ }_{\star} K_{h} / h_{\star} K_{h}$ is exactly isomorphic (as a left bialgebroid over $A$ ) to $V^{\ell}(L)$. Now we show that the structure of Lie-Rinehart bialgebra induced on $L$ by the quantization ${ }_{\star} K_{h}$ of $V^{\ell}(L)$ is the same as that induced by the quantization $K_{h}$ of $J^{r}(L)$. To this end, let $[,]^{\prime}, \omega^{\prime}$, be the Lie bracket and the anchor map on $L^{*}$ induced by ${ }_{\star} K_{h}$, and [, ] $]^{\prime \prime}, \omega^{\prime \prime}$, those induced by $K_{h}$.

We proceed like in the proof of Theorem 5.1.5. Our goal is to prove that $\omega^{\prime}=\omega^{\prime \prime}$ and $[,]^{\prime}=[,]^{\prime \prime}$; thus recall that (cf. Remarks 2.2.2 (b)) $\omega^{\prime}$ and $[,]^{\prime}$ are uniquely determined by

$$
\begin{aligned}
\omega^{\prime}(\Phi)(a) & =\left\langle\delta_{L}(a), \Phi\right\rangle, \\
\left\langle\Theta,[\Phi, \Psi]^{\prime}\right\rangle & =\omega^{\prime}(\Phi)(\langle\Theta, \Psi\rangle)-\omega^{\prime}(\Psi)(\langle\Theta, \Phi\rangle)-\left\langle\delta_{L}(\Theta), \Phi \otimes \Psi\right\rangle
\end{aligned}
$$

(for all $\Phi, \Psi \in L^{*}, \Theta \in L, a \in A$ ), where $\delta_{L}(a)$ and $\delta_{L}(\Theta)$ are defined by the formula for $\delta$ in Theorem 4.1.3. Thus it is enough to prove that (for all $\Phi, \Psi \in L^{*}$, $\Theta \in L, a \in A$ )

$$
\begin{aligned}
\omega^{\prime \prime}(\Phi)(a) & =\left\langle\delta_{L}(a), \Phi\right\rangle, \\
\left\langle\Theta,[\Phi, \Psi]^{\prime \prime}\right\rangle & =\omega^{\prime}(\Phi)(\langle\Theta, \Psi\rangle)-\omega^{\prime}(\Psi)(\langle\Theta, \Phi\rangle)-\left\langle\delta_{L}(\Theta), \Phi \otimes \Psi\right\rangle
\end{aligned}
$$

To prove (5.7), choose liftings $\phi^{\prime}, \psi^{\prime} \in J^{r}(L)_{h}=: K_{h}$, with the additional condition that $\phi^{\prime}, \psi^{\prime} \in \mathfrak{J}_{J^{r}(L)_{h}}:=\operatorname{Ker}\left(\partial_{J^{r}(L)_{h}}\right)$ (this is always possible), a lifting $\theta \in V^{\ell}(L)_{h}:={ }_{\star} J^{r}(L)_{h}$ of $\Theta$ and a lifting $a^{\prime} \in A_{h}$ of $a$. Now direct computation gives

$$
\begin{aligned}
\omega^{\prime}(\Phi)(a) & =\left\langle\delta_{L}(a), \Phi\right\rangle \\
& =h^{-1}\left\langle t_{*}^{\ell}\left(a^{\prime}\right)-s_{*}^{\ell}\left(a^{\prime}\right), \phi^{\prime}\right\rangle \bmod h A_{h} \\
& =h^{-1}\left\langle 1, \phi^{\prime} s_{r}\left(a^{\prime}\right)-s_{r}\left(a^{\prime}\right) \phi^{\prime}\right\rangle \bmod h A_{h} \\
& =\left(\frac{\phi^{\prime} s_{r}\left(a^{\prime}\right)-s_{r}\left(a^{\prime}\right) \phi^{\prime}}{h} \bmod h K_{h}\right) \bmod \mathfrak{J}_{r^{r}(L)} \\
& =\omega^{\prime \prime}(\Phi)(a)
\end{aligned}
$$


where we exploited the fact that the involved pairing a right bialgebroid pairing (cf. Definitions 3.4.4 and 3.4.5). Thus the first identity in (5.7) is verified.

As to the rest, we write $\Delta(\theta)=\theta_{(1)} \otimes \theta_{(2)}$ as $\Delta(\theta)=\theta \otimes 1+1 \otimes \theta+h \theta_{[1]} \otimes \theta_{[2]}$, so that $\left(\theta_{[1]} \otimes \theta_{[2]}\right) \bmod h V^{\ell}(L)_{h \triangleleft \times_{\triangleright}} V^{\ell}(L)_{h}=: \Delta^{[1]}(\Theta)$, as in Definition 4.1.3. Moreover, let us set $\phi:=\phi^{\prime} \bmod h J^{r}(L)_{h}, \psi:=\psi^{\prime} \bmod h J^{r}(L)_{h}$, which are lifts of $\Phi$ and $\Psi$ in $J^{r}(L)$, and actually belong to $\mathfrak{J}_{J} r_{(L)}$. Then direct computation gives

$$
\left\langle\Theta,[\Phi, \Psi]^{\prime \prime}\right\rangle=\langle\Theta,\{\phi, \psi\}\rangle=\left\langle\theta, \frac{\phi^{\prime} \psi^{\prime}-\psi^{\prime} \phi^{\prime}}{h}\right\rangle \bmod h A_{h}
$$

Now, in the proof of Theorem 5.1.5 - namely, to prove the second part of (5.6) we saw that

$$
\left\langle\theta, \frac{\phi^{\prime} \psi^{\prime}-\psi^{\prime} \phi^{\prime}}{h}\right\rangle \bmod h A_{h}=\omega^{\prime}(\Phi)(|\Theta, \Psi\rangle)-\omega^{\prime}(\Psi)\left(|\Theta, \Phi\rangle-\left\langle\delta_{L}(\Theta), \Phi \otimes \Psi\right\rangle\right.
$$

so that the second identity in (5.7) is proved.

At last, let now cope with the case of ${ }^{\star} K_{h}$. Clearly, we can proceed much like for ${ }_{\star} K_{h}$ : one proves that ${ }^{\star} K_{h}={ }^{\star} J^{r}(L)_{h}$ is a topological left bialgebroid, whose specialization modulo $h$ is ${ }^{\star} J^{r}(L) \cong V^{\ell}(L)$, hence we can claim that ${ }^{\star} J^{r}(L)_{h} \in$ (LQUEAd) $A_{h}$ is a quantization of $V^{\ell}(L)$.

A difference arises about the last part of claim (a), concerning the two LieRinehart algebra structures induced on $L^{*}$ : indeed, the difference in the outcome - a minus sign - is due to the fact that the natural pairing (given by evaluation) among the left bialgebroid ${ }^{\star} J^{r}(L)_{h}$ and the right bialgebroid $J^{r}(L)_{h}$ is now a left bialgebroid pairing (cf. Definitions 3.4.4 and 3.4.5) - while for ${ }_{\star} J^{r}(L)_{h}$ and $J^{r}(L)_{h}$ it is a right one. Explicit computations are (again) much like those in the proof of Theorem 5.1.5 (for the very last part of claim (a)), just as it occurs for ${ }_{\star} K_{h}={ }_{\star} J^{r}(L)_{h}$.

(b) The arguments used to prove claim (a) clearly adapt to claim (b) as well. Otherwise, one can deduce (b) directly from claim (a) using general isomorphisms such as $\star\left(U_{\text {coop }}^{o p}\right) \cong\left(U^{\star}\right)_{\text {coop }}^{o p}$ and ${ }^{\star}\left(U_{\text {coop }}^{o p}\right) \cong\left(U_{\star}\right)_{\text {coop }}^{o p}$ - see Remark 3.4.6.

5.3. Functoriality of linear duality for quantum groupoids. The results in Sections 5.1 and 5.2 about the duality constructions for quantum bialgebroids can be improved. Indeed, they can be cast in the following, functorial version (cf. Definition 4.1.1 and 4.1.8 for notation), which is the main outcome of this section: 
Theorem 5.3.1. Left and right duals yield pairs of well-defined contravariant functors

$$
\begin{aligned}
& \left(\text { LQUEAd } A _ { h } \longrightarrow \left(\text { RQFSAd }_{A_{h}}, \quad H_{h} \mapsto H_{h}^{*},\right.\right.
\end{aligned}
$$

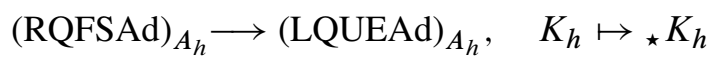

$$
\begin{aligned}
& (\text { LQUEAd })_{A_{h}} \longrightarrow\left(\text { RQFSAd }_{A_{h}}, \quad H_{h} \mapsto H_{h_{*}}\right. \text {, } \\
& (\text { RQFSAd })_{A_{h}} \longrightarrow(\text { LQUEAd })_{A_{h}}, \quad K_{h} \mapsto{ }^{\star} K_{h} \\
& (\text { RQUEAd })_{A_{h}} \longrightarrow(\text { LQFSAd })_{A_{h}}, \quad H_{h} \mapsto{ }^{*} H_{h}, \\
& \left(\text { LQFSAd } A _ { h } \longrightarrow \left(\text { RQUEAd } A_{A_{h}}, \quad K_{h} \mapsto K_{h_{\star}}\right.\right. \\
& (\text { RQUEAd })_{A_{h}} \longrightarrow\left(\text { LQFSAd } A_{A_{h}}, \quad H_{h} \mapsto{ }_{*} H_{h},\right. \\
& \left(\text { LQFSAd } _ { A _ { h } } \longrightarrow \left(\text { RQUEAd) } A_{h}, \quad K_{h} \mapsto K_{h}^{\star}\right.\right.
\end{aligned}
$$

which are (pairwise) inverse to each other, hence yield pairs of antiequivalences of categories.

Proof. It is clearly enough to present the proof for just one pair of functors, say those in first line.

Let $H_{h}=V^{\ell}(L)_{h} \in(\text { LQUEAd) })_{A_{h}}$. For any $\lambda \in H_{h}^{*}$ and any $\eta \in H_{h}$, let $e v_{\eta}(\lambda):=\lambda(\eta)$, and consider the map $H_{h} \longrightarrow{ }_{\star}\left(H_{h}^{*}\right)$ given by $\eta \mapsto e v_{\eta}$; note that a priori this map takes values in $*\left(H_{h}^{*}\right)$, but Lemma 5.1.4 actually proves that every $e v_{\eta}$ belongs to $\star\left(H^{*}\right)$.

Now, this map is an isomorphism in (LQUEAd) ${ }_{A_{h}}$ because it is an isomorphism modulo $h$. The other points can also be proved, by standard arguments, in a similar way.

\section{Drinfeld's functors and quantum duality}

In this section we present the main new contribution in this paper, namely the definition of Drinfeld's functors and the equivalences - instead of antiequivalences! — of categories established via them among (left or right) QUEAd's and QFSAd's.

\subsection{The Drinfeld's functor ()$^{\vee}$.}

Definition 6.1.1. Let $K_{h} \in(\mathrm{RQFSAd})_{A_{h}}$. We set $I_{h}:=\partial_{h}^{-1}\left(h A_{h}\right)$ and $\mathfrak{J}_{h}:=\operatorname{Ker}\left(\partial_{h}\right)$, where $\partial_{h}$ is the counit of $K_{h}$; then one has $I_{h}=\mathfrak{J}_{h}+h K_{h}$. We define

$$
K_{h}^{\times}:=s^{r}\left(A_{h}\right)+\sum_{n \in \mathbb{N}_{+}} h^{-n} I_{h}^{n}=s^{r}\left(A_{h}\right)+\sum_{n \in \mathbb{N}_{+}} h^{-n} s^{r}\left(A_{h}\right) \mathfrak{J}_{h}^{n},
$$


which is a $k[[h]]-$ submodule of $K_{F}:=k((h)) \underset{k[[h]]}{\otimes} K_{h}$, and we denote by $K_{h}^{\vee}$ the $h$-adic completion of the $k[[h]]-$ module $K_{h}^{\times}$.

Moreover, in an entirely similar way we define $K_{h}^{\vee}$ for any $K_{h} \in(\mathrm{LQFSAd})_{A_{h}}$.

Remarks 6.1.2. (a) Note that $\mathfrak{J}_{h}$ is not an $\left(A_{h}, A_{h}\right)$-subbimodule of $K_{h}$, in general. Indeed, if $a \in A_{h}$ and $\psi \in \mathfrak{J}_{h}$, it is clear (from the properties of the counit of a right bialgebroid) that $\psi s_{r}(a), \psi t_{r}(a)$ in $\mathfrak{J}_{h}$; but we cannot prove in general that $s_{r}(a) \psi$ and $t_{r}(a) \psi$ belong to $\mathfrak{J}_{h}$. On the other hand, one has that $I_{h}$ instead is definitely an $\left(A_{h}, A_{h}\right)$-subbimodule. For this reason, it is better to (define and) describe $K_{h}^{\times}$and $K_{h}^{\vee}$ using $I_{h}$ than using $\mathfrak{J}_{h}$.

(b) Let $K$ be a LQFSAd, respectively a RQFSAd. Then $\left(K_{h}\right)_{\text {coop }}^{o p}$ is a RQFSAd, respectively a LQFSAd. It easily follows from definitions that $\left(\left(K_{h}\right)_{\text {coop }}^{o p}\right)^{\vee}=$ $\left(K_{h}^{\vee}\right)_{\text {coop }}^{\text {op }}$

6.1.3. Description of $K_{h}^{\vee}$. Directly from its very definition, we can find out a description of $K_{h}^{\vee}$. This is very neat in the case when the Lie-Rinehart algebra $L-$ such that $K_{h}$ is a quantization of $J^{r}(L)$ or $J^{\ell}(L)$ - is of finite free type (as an $A$-module), and can be reduced somehow to that case when $L$ instead is just of finite projective type. Thus we distinguish these two cases.

(a) Finite free case: Let us assume that $L$ (as an $A$-module, of finite type) is free. Then we can explicitly describe $K_{h}^{\vee}$, as follows. Fix an $A$-basis $\left\{\bar{e}_{1}, \ldots, \bar{e}_{n}\right\}$ of $L$, and let $\bar{\xi}_{i}$ be the element of $\operatorname{Hom}\left(V^{\ell}(L), A\right)=V^{\ell}(L)^{*}=J^{r}(L)$ defined (using standard multiindex notation) by

$$
\left\langle\bar{\xi}_{i}, \bar{e}^{\underline{\alpha}}\right\rangle=\left\langle\bar{\xi}_{i}, \bar{e}_{1}^{\alpha_{1}} \cdots \bar{e}_{n}^{\alpha_{n}}\right\rangle:=\delta_{\alpha_{1}, 0} \cdots \delta_{\alpha_{i}, 1} \cdots \delta_{\alpha_{n}, 0} \quad \forall \underline{\alpha}=\left(\alpha_{1}, \ldots, \alpha_{n}\right) \in \mathbb{N}^{n}
$$

Let $\xi_{i}$ be an element of $K_{h}$ lifting $\bar{\xi}_{i}$ and such that $\partial_{h}\left(\xi_{i}\right)=0$. If $a \in A_{h}$, we shall write again $a$ to denote the element $t^{r}(a) \in K_{h}$. We have the following descriptions

$$
\begin{aligned}
K_{h} & =\left\{\sum_{\underline{d} \in \mathbb{N}^{n}} \xi_{1}^{d_{1}} \ldots \xi_{n}^{d_{n}} a_{\underline{d}} \mid a_{\underline{d}} \in A_{h}^{o p}, \forall \underline{d} \in \mathbb{N}^{n}\right\} \cong A\left[\left[X_{1}, \ldots, X_{n}\right]\right][[h]] \\
I_{h} & =\left(h, \xi_{1}, \ldots, \xi_{n}\right), \\
\mathfrak{J}_{h} & =\sum_{i=1}^{n} \xi_{i} K_{h}
\end{aligned}
$$

where the first line item is a (right) $A_{h}^{o p}$-module of formal power series (convergent in the $I_{h}$-adic topology) and the last isomorphism is one of topological $k$-modules, while round braces in second line stand once again for "two-sided ideal generated by". By this and the very definition it follows that, writing $\check{\xi}_{i}:=h^{-1} \xi_{i}$, one has (the last isomorphism being one of topological $k$-modules)

$$
K_{h}^{\vee}=\left\{\sum_{\underline{b} \in \mathbb{N}^{n+1}} h^{b_{0}} \check{\xi}_{1}^{b_{1}} \ldots \check{\xi}_{n}^{b_{n}} a_{\underline{b}} \mid a_{\underline{b}} \in A_{h}^{o p}, \forall \underline{b}\right\} \cong A\left[\check{X}_{1}, \ldots, \check{X}_{n}\right][[h]]
$$

where the sum denotes formal series which are convergent in the $h$-adic topology, and then also

$$
\mathfrak{J}_{h}^{\vee}:=h^{-1} \mathfrak{J}_{h}=\sum_{i=1}^{n} \check{\xi}_{i} K_{h}^{\vee}=\text { right ideal of } K_{h}^{\vee} \text { generated by the } \check{\xi}_{i} \text { 's }
$$


(b) Finite projective case: Assume now that $L$ (as an $A$-module) is just finite projective (as usual in this work). Like in Subsection 4.2, we fix a finite projective $A$-module $Q$ such that $L \oplus Q=F$ is a finite free $A$-module, we write $F=A \otimes_{k} Y$ where $Y$ is the $k$-span of an $A$-basis of $F$, and we construct the (infinite dimensional) Lie-Rinehart algebra $L_{Q}=L \oplus\left(A \otimes_{k} Z\right)$ with $Z=Y \oplus Y \oplus Y \oplus \cdots$. Then, for $J^{r}(L)_{h}:=K_{h}$, we can introduce the right bialgebroid $K_{h, Y}:=J^{r}(L)_{h, Y}$ as in §4.2.3: namely (with notation as in §4.2.3), we recall that

$$
K_{h, Y}:=h \text {-adic completion of } \sum_{n \in \mathbb{N}} K_{h} \widetilde{\otimes}_{k} S\left(Y^{*}\right)^{\widetilde{\otimes} n} \otimes 1 \otimes 1 \otimes 1 \cdots
$$

$\left(S\left(Y^{*}\right)^{\otimes n}\right)^{+}$being the kernel of the natural counit map of $S\left(Y^{*}\right)^{\otimes n}$ and $K_{h} \widetilde{\otimes}_{k} S\left(Y^{*}\right)^{\otimes n}$ $\otimes 1 \cdots$ the $\left(\left(S\left(Y^{*}\right)^{\otimes n}\right)^{+} \otimes 1 \otimes 1 \cdots\right)$-adic completion of $K_{h} \otimes_{k} S\left(Y^{*}\right)^{\otimes n} \otimes 1 \otimes 1 \cdots$.

Furthermore, let $\partial_{h}$ be the counit of $K_{h, Y}$, and $I_{h, Y}:=\partial_{h}^{-1}\left(h A_{h}\right)$. Then we have also

$$
\begin{aligned}
& I_{h, Y}:=h \text {-adic completion of } \\
& \qquad\left(\sum_{r \in \mathbb{N}} I_{h} \underset{k}{\widetilde{\otimes}} S\left(Y^{*}\right)^{\widetilde{\otimes} r} \otimes 1 \otimes \cdots+\sum_{s \in \mathbb{N}} K_{h} \underset{k}{\widetilde{\otimes}}\left(S\left(Y^{*}\right)^{\widetilde{\otimes} s}\right)^{+} \otimes 1 \otimes \cdots\right)
\end{aligned}
$$

Basing upon these remarks, we can define $K_{h, Y}^{\vee}$ and describe it as above: namely, one has

$$
\begin{aligned}
K_{h, Y}^{\vee} & =h \text {-adic completion of } \sum_{n, m r+s=n} h^{-n} \mathfrak{J}_{h}^{r} \widetilde{\otimes}_{k}\left(\left(S\left(Y^{*}\right)^{\widetilde{\otimes} m}\right)^{+}\right)^{s} \otimes 1 \otimes 1 \otimes \cdots \\
& =K_{h}^{\vee} \widehat{\otimes}_{k} S\left(Z^{* f}\right)
\end{aligned}
$$

where $Z^{*}=Y^{*} \oplus Y^{*} \oplus Y^{*} \oplus \cdots$.

Let now $\left\{e_{t}\right\}_{t \in T:=\mathbb{N} \times\{1, \ldots, n\}}$ be a good basis of the $A$-module $L_{Q}$. From the proof of Theorem 5.2.2 (step (4) for the general case) we can select elements $\xi_{t} \in$ $K_{h, Y}(t \in T)$ such that

$$
\begin{aligned}
K_{h, Y} & =\left\{\sum_{n \in \mathbb{N}} h^{n} P_{n}\left(\left\{\xi_{t}\right\}_{t \in T}\right) \mid P_{n} \in\left[\left[\left\{X_{t}\right\}_{t \in T}\right]\right]_{f} A_{h}^{o p}\right\} \cong A\left[\left[\left\{X_{t}\right\}_{t \in T}\right]\right]_{f}[[h]] \\
I_{h, Y} & =\left(h,\left\{\xi_{t}\right\}_{t \in T}\right), \\
\mathfrak{J}_{h, Y} & =\sum_{t \in T} \xi_{t} K_{h, Y}
\end{aligned}
$$

where $\left[\left[\left\{X_{t}\right\}_{t \in T}\right]\right]_{f} A_{h}$ is the ring of formal power series with coefficients on the right chosen in $A_{h}$ involving only finitely many indeterminates $X_{t}$. One easily finds, letting $\check{\xi}_{t}:=h^{-1} \xi_{t}$, that

$$
K_{h, Y}^{\vee}=\left\{\sum_{n \in \mathbb{N}} h^{n} P_{n}\left(\left\{\check{\xi}_{t}\right\}_{t \in T}\right) \mid P_{n} \in\left[\{\underline{\xi}\}_{t \in T}\right] A_{h}^{o p}, \forall n \in \mathbb{N}\right\} \cong A\left[\left\{\check{X}_{t}\right\}_{t \in T}\right][[h]]
$$


where the sum denotes formal series convergent in the $h$-adic topology, and $\left[\left\{X_{t}\right\}_{t \in T}\right] A_{h}^{o p}$ denotes the ring of polynomials with coefficients on the right chosen in $A_{h}^{o p}$. We find also

$$
\mathfrak{J}_{h, Y}^{\vee}:=h^{-1} \mathfrak{J}_{h, Y}=\sum_{t \in T} \check{\xi}_{t} K_{h, Y}^{\vee}=\text { right ideal of } K_{h, Y}^{\vee} \text { generated by the } \check{\xi}_{t} \text { 's } \diamond
$$

It is time for the main result of this subsection. In short, it claims that the construction $K_{h} \mapsto K_{h}^{\vee}$, starting from a quantization of $L-$ of type $J^{r / \ell}(L)-$ provides a quantization of the dual Lie-Rinehart bialgebra $L^{*}$ - of type $V^{r / \ell}\left(L^{*}\right)$; moreover, this construction is functorial.

Theorem 6.1.4. (a) Let $J^{r}(L)_{h} \in(\mathrm{RQFSAd})_{A_{h}}$, where $L$ is a Lie-Rinehart algebra which, as an A-module, is projective of finite type. Then:

a.1 $J^{r}(L)_{h}^{\vee} \in\left(\right.$ RQUEAd) ${ }_{A_{h}}$, with semiclassical limit $J^{r}(L)_{h}^{\vee} / h J^{r}(L)_{h}^{\vee} \cong$ $V^{r}\left(L^{*}\right)$. Moreover, the structure of Lie-Rinehart bialgebra induced on $L^{*}$ by the quantization $J^{r}(L)_{h}^{\vee}$ of $V^{r}\left(L^{*}\right)$ - as in Theorem 4.1.6-is dual to that induced on $L$ by the quantization $J^{r}(L)_{h}$ of $J^{r}(L)$ - as in Theorem 4.1.12;

a.2 the definition of $J^{r}(L)_{h} \mapsto J^{r}(L)_{h}^{\vee}$ extends to morphisms in (RQFSAd), so that we have a well defined (covariant) functor ()$^{\vee}:($ RQFSAd $) \longrightarrow$ (RQUEAd).

(b) Let $J^{\ell}(L)_{h} \in(\mathrm{LQFSAd})_{A_{h}}$, where L is a Lie-Rinehart algebra which, as an A-module, is projective of finite type. Then:

b.1 $J^{\ell}(L)_{h}^{\vee} \in(\text { LQUEAd })_{A_{h}}$, with semiclassical limit $J^{\ell}(L)_{h}^{\vee} / h J^{\ell}(L)_{h}^{\vee} \cong$ $V^{\ell}\left(L^{*}\right)$. Moreover, the structure of Lie-Rinehart bialgebra induced on $L^{*}$ by the quantization $J^{\ell}(L)_{h}^{\vee}$ of $V^{\ell}\left(L^{*}\right)$ - as in Theorem 4.1.3 - is dual to that induced on $L$ by the quantization $J^{\ell}(L)_{h}$ of $J^{\ell}(L)$ - as in Theorem 4.1.13;

b.2 the definition of $J^{\ell}(L)_{h} \mapsto J^{\ell}(L)_{h}^{\vee}$ extends to morphisms in (LQFSAd), so that we have a well defined (covariant) functor ()$^{\vee}:$ (LQFSAd) $\longrightarrow$ (LQUEAd).

Proof. (a) In order to ease notation, let us write $K_{h}:=J^{r}(L)_{h}$.

By definition, $K_{h}^{\times}$is the unital $k[[h]]-$ subalgebra of $\left(K_{h}\right)_{F}:=k((h)) \otimes_{k[[h]]} K_{h}$ generated by $h^{-1} I_{h}$ and $s^{r}\left(A_{h}\right)$ : thus it is automatically a unital $k[[h]]-$ algebra. It follows that $K_{h}^{\vee}$ is a unital $k[[h]]-$ algebra too, complete in the $h$-adic topology. Moreover, $I_{h}$ is an $\left(A_{h}, A_{h}\right)$-subbimodule of $K_{h}$ : this implies at once that $K_{h}^{\times}$ and $K_{h}^{\vee}$ are $\left(A_{h}, A_{h}\right)$-bimodules too. As $\left(K_{h}\right)_{F}$ is torsionless, so are $K_{h}^{\times}$and its completion $K_{h}^{\vee}$; also, $K_{h}^{\vee}$ is separated and complete, so it is topologically free.

Let us now see that the coproduct in $K_{h}$ induces a coproduct - in a suitable, $h-$ adical sense - for $K_{h}^{\vee}$ as well. Given any $\phi \in I_{h}$, we write $\Delta(\phi)=\phi_{(1)} \otimes \phi_{(2)}-\mathrm{a}$ formal series (in $\Sigma$-notation) - convergent in the $I_{h}$-adic topology of $K_{h}$. Writing $\phi_{(1)}$ and $\phi_{(2)}$ as

$$
\phi_{(1)}=\phi_{(1)}^{+s}+s^{r}\left(\partial_{h}\left(\phi_{(1)}\right)\right), \quad \phi_{(2)}=\phi_{(2)}^{+t}+t^{r}\left(\partial_{h}\left(\phi_{(2)}\right)\right)
$$


we have seen that $\Delta(\phi)=\phi_{(1)}^{+s} \otimes \phi_{(2)}+s^{r}\left(\partial_{h}\left(\phi_{(1)}\right)\right) \otimes \phi_{(2)}^{+t}+s^{r}\left(\partial_{h}(\phi)\right) \otimes 1$ belongs to the space $\left(I_{h} \widetilde{\otimes}_{A_{h}} K_{h}+K_{h} \widetilde{\otimes}_{A_{h}} I_{h}+h s^{r}\left(A_{h}\right) \widetilde{\otimes}_{A_{h}} 1\right)$. All this implies $\Delta\left(h^{-1} \phi\right) \in K_{h}^{\vee} \widetilde{\otimes}_{A_{h}} K_{h}^{\vee}$.

In addition, we must observe the following. Every $\phi \in I_{h}$ expands as an $I_{h}$-adically convergent series $\phi=\sum_{n \in \mathbb{N}_{+}} \phi_{n}$ with $\phi_{n} \in I_{h}^{n}$ for all $n \in \mathbb{N}_{+}$; but then $\phi_{n} \in I_{h}^{n}=h^{n}\left(h^{-1} I_{h}\right)^{n}$ for every $n$ and so $h^{-1} \phi$ expands as a series $h^{-1} \phi=\sum_{n \in \mathbb{N}_{+}} h^{n-1}\left(h^{-1} I_{h}\right)^{n}$ which is convergent in the $h$-adic topology of $K_{h}^{\vee}$. As a byproduct of this analysis, we can apply the same argument to $\Delta\left(h^{-1} \phi\right)$ and thus realize that it is actually a well defined element of $K_{h}^{\vee} \widehat{\otimes}_{A_{h}} K_{h}^{\vee}$, the $h$-adic completion of $K_{h}^{\vee} \otimes_{A_{h}} K_{h}^{\vee}$. Finally, it is clear that in fact $\Delta\left(h^{-1} \phi\right)$ even belongs to the Takeuchi product inside $K_{h}^{\vee} \otimes_{A_{h}} K_{h}^{\vee}$, as the parallel property is true for $\Delta(\phi)$ inside $K_{h} \widetilde{\otimes}_{A_{h}} K_{h}$.

As $K^{\times}$is generated - as an algebra - by $h^{-1} I_{h}$ and $s^{r}\left(A_{h}\right)$, and $K_{h}^{\vee}$ is its $h$-adic completion, we finally conclude that the coproduct of $K_{h}$ does provide a well defined coproduct for $K_{h}^{\vee}$, making it into a (topological) right bialgebroid over $A_{h}$.

Moreover, by construction $K_{h}^{\vee}$ is isomorphic (as a $k[[h]]-$ module) to $\left(K_{h}^{\vee} / h K_{h}^{\vee}\right)[[h]]$.

What we are left to prove - for claim (a.1) - is that $\overline{K_{h}^{\vee}}:=K_{h}^{\vee} / h K_{h}^{\vee}$ be isomorphic to $V^{r}\left(L^{\prime}\right)$ for some Lie-Rinehart bialgebra, and that such $L^{\prime}$ - with its structure of (Lie-Rinehart) bialgebra induced by this very quantization - is isomorphic to $L^{*}$ with its structure of Lie-Rinehart bialgebra dual to that induced on $L$ by the quantization $K_{h}:=J^{r}(L)_{h}$ we started from.

We follow the strategy in [12] and [9]. So far we saw that $K_{h}^{\vee}$ is a deformation of the right bialgebroid $K_{h}^{\vee} / h K_{h}^{\vee}$ : then we shall apply Proposition 3.3.4 (and the remarks after it) to show that the latter is indeed of the form $V^{r}\left(L^{\prime}\right)$, with $L^{\prime} \cong L^{*}$. For computations hereafter we fix some notation: $\mathfrak{J}_{h}:=\operatorname{Ker}\left(\partial_{h}\right), K:=K_{h} / h K_{h}$ and $\mathfrak{J}:=\operatorname{Ker}(\partial)$ for $\partial:=\partial_{K}$. Also, from Theorem 5.2.2 (a) we consider $V^{\ell}(L)_{h}:=$ ${ }_{\star} K_{h}={ }_{\star} J^{r}(L)_{h} \in(\text { LQUEAd })_{h}$ so that $J^{r}(L)_{h}=V^{\ell}(L)_{h}^{*}$.

We proceed in several steps.

- For all $a \in A_{h}$, we have $s_{r}(a) \equiv t_{r}(a) \bmod h K_{h}^{\vee}$. Indeed, one has $\left(s^{r}(a)-\right.$ $\left.t^{r}(a)\right) \in \mathfrak{J}_{h} \subseteq I_{h}=h h^{-1} I_{h} \subseteq h K_{h}^{\vee}$, whence the claim.

- The set $P^{r}\left(\overline{K_{h}^{\vee}}\right)$ of (right) primitive elements of $\overline{K_{h}^{\vee}}:=K_{h}^{\vee} / h K_{h}^{\vee}-$ cf. Proposition 3.3.4 - has a natural structure of right Lie-Rinehart algebra, induced by specialization from $K_{h}^{\vee}$.

Indeed, this is entirely standard. Both the Lie bracket [, ] and the anchor map $\omega$ are recovered as semiclassical limits of commutators from the multiplicative structure and source/target structure of the "quantum right bialgebroid" $K_{h}^{\vee}$. Namely, for any $x, y \in P^{r}\left(\overline{K_{h}^{\vee}}\right)$ and $a \in A$, choose any lifts $x^{\prime}, y^{\prime} \in K_{h}^{\vee}$ and $a^{\prime} \in A_{h}$ of 
them: then defining

$$
\begin{gathered}
a \cdot x:=x^{\prime} s^{r}\left(a^{\prime}\right) \quad \bmod h K_{h}^{\vee}, \quad[x, y]:=x^{\prime} y^{\prime}-y^{\prime} x^{\prime} \quad \bmod h K_{h}^{\vee} \\
\omega(x)(a):=\partial_{h}\left(x^{\prime} s^{r}\left(a^{\prime}\right)-s^{r}\left(a^{\prime}\right) x^{\prime}\right) \quad \bmod h A_{h}
\end{gathered}
$$

it is a routine matter to check that $P^{r}\left(\overline{K_{h}^{\vee}}\right)$ is made into a Lie-Rinehart algebra over $A$.

- Set $\mathfrak{J}_{h}^{\vee}:=h^{-1} \mathfrak{J}_{h}\left(\subseteq K_{h}^{\vee}\right)$ and $\overline{\mathfrak{J}_{h}^{\vee}}:=\mathfrak{J}_{h}^{\vee} \bmod h K_{h}^{\vee}$; then $\overline{\mathfrak{J}_{h}^{\vee}}$ is a LieRinehart subalgebra of $P^{r}\left(\overline{K_{h}^{\vee}}\right)$.

Indeed, let $\phi \in \mathfrak{J}_{h}$, and set $\phi^{\vee}:=h^{-1} \phi \in \mathfrak{J}_{h}^{\vee}$. Then acting as in the first part of the proof (with notation introduced therein) we get

$$
\Delta(\phi)=\phi \otimes 1+1 \otimes \phi+\phi_{(1)}^{+s} \otimes \phi_{(2)}^{+t} \in \phi \otimes 1+1 \otimes \phi+\mathfrak{J}_{h} \widetilde{\otimes}_{A_{h}} \mathfrak{J}_{h}
$$

thanks to the assumption $\phi \in \mathfrak{J}_{h}$ (and to several identities holding true in any right bialgebroid). As $\mathfrak{J}_{h}=h h^{-1} \mathfrak{J}_{h}=h \mathfrak{J}_{h}^{\vee} \subseteq h K_{h}^{\vee}$, we end up with $\Delta\left(\phi^{\vee}\right)=\phi^{\vee} \otimes 1+$ $1 \otimes \phi^{\vee}+h\left(K_{h}^{\vee} \widehat{\otimes}_{A_{h}} K_{h}^{\vee}\right)$, so that $\overline{\phi^{\vee}}:=\phi^{\vee} \bmod h K_{h}^{\vee}$ is primitive in $\overline{K_{h}^{\vee}}$. This proves that $\overline{\mathfrak{J}_{h}^{\vee}} \subseteq P^{r}\left(\frac{K_{h}^{\vee}}{)}\right.$.

Finally, $\overline{\mathfrak{J}_{h}^{\vee}}$ is a Lie-Rinehart subalgebra of $P^{r}\left(\overline{K_{h}^{\vee}}\right)$ if and only if it is a (right) $A$-submodule, closed for the Lie bracket. Now, by definition $\mathfrak{J}_{h}$ is a right ideal in $K_{h}$, and this implies - by construction - that $\overline{\mathfrak{J}_{h}^{\vee}}$ is a (right) $A$-submodule. As to the Lie bracket, if $x, y \in \overline{\mathfrak{J}_{h}^{\vee}}$ we have by definition $[x, y]:=x^{\prime} y^{\prime}-y^{\prime} x^{\prime} \bmod h K_{h}^{\vee}$ for any choice of liftings $x^{\prime}, y^{\prime} \in K_{h}^{\vee}$ of $x$ and $y$. On the other hand, we can clearly choose $x^{\prime}, y^{\prime} \in \mathfrak{J}_{h}^{\vee}$, so that $x^{\prime}=h^{-1} \chi, y^{\prime}=h^{-1} \eta$, for some $\chi, \eta \in \mathfrak{J}_{h}$; then we have

$$
x^{\prime} y^{\prime}-y^{\prime} x^{\prime}=h^{-2}(\chi \eta-\eta \chi) \in h^{-2}\left(\mathfrak{J}_{h} \bigcap h K_{h}\right)=h^{-2} h \mathfrak{J}_{h}=h^{-1} \mathfrak{J}_{h}=: \mathfrak{J}_{h}^{\vee}
$$

since $\mathfrak{J}_{h}$ is a right ideal and $K_{h} / h K_{h} \cong J^{r}(L)$ is commutative. It follows that $[x, y] \in \overline{\mathfrak{J}_{h}^{\vee}}$, q.e.d.

- We will now show that $\mathfrak{J}_{h}^{\vee} \bigcap h K_{h}^{\vee}=\mathfrak{J}_{h}+\mathfrak{J}_{h}^{\vee} \mathfrak{J}_{h}=h \mathfrak{J}_{h}^{\vee}+h\left(\mathfrak{J}_{h}^{\vee}\right)^{2}$.

Indeed, the second identity in the claim is a trivial consequence of $\mathfrak{J}_{h}^{\vee}:=h^{-1} \mathfrak{J}_{h}$. As to the first one, as $K_{h}=J^{r}(L)_{h}$, we distinguish two cases: either $L$ is free (as an $A$-module), or not.

If $L$ is free, then the identity $\mathfrak{J}_{h}^{\vee} h h K_{h}^{\vee}=\mathfrak{J}_{h}+\mathfrak{J}_{h}^{\vee} \mathfrak{J}_{h}$ is an easy, direct consequence of the description of $\mathfrak{J}_{h}^{\vee}$ given in $\$ 6.1 .3$ here above in the free case -i.e. $\operatorname{part}(\mathrm{a})$.

If instead $L$ is not free, then we proceed as follows. First consider $K_{h, Y}$ and $\mathfrak{J}_{h, Y}$, and construct from them $K_{h, Y}^{\vee}$ and $\mathfrak{J}_{h, Y}^{\vee}$. In this case, the description of $\mathfrak{J}_{h, Y}^{\vee}$ given in \$6.1.3, part (b), implies again easily the identity $\mathfrak{J}_{h, Y}^{\vee} \bigcap h K_{h, Y}^{\vee}=\mathfrak{J}_{h, Y}+\mathfrak{J}_{h, Y}^{\vee} \mathfrak{J}_{h, Y}$. Now consider the map $\pi^{Y}: K_{h, Y} \longrightarrow K_{h}$, introduced in $\S 4.2 .3(\mathrm{~b})$, for $J^{r}(L)_{h}:=$ $K_{h}$ and $J^{r}(L)_{h, Y}:=K_{h, Y}$ : this is a an epimorphism of right bialgebroids, thus 
in particular $\pi^{Y}\left(\mathfrak{J}_{h, Y}\right)=\mathfrak{J}_{h}$. Then it follows at once that $\pi^{Y}$ canonically induces another epimorphism of right bialgebroids $\check{\pi}^{Y}: K_{h, Y}^{\vee} \longrightarrow K_{h}^{\vee}$ such that $\check{\pi}^{Y}\left(\mathfrak{J}_{h, Y}^{\vee}\right)=$ $\mathfrak{J}_{h}^{\vee}$. But then, using $\pi^{Y}$ and $\check{\pi}^{Y}$ and the identity $\mathfrak{J}_{h, Y}^{\vee} \cap h K_{h, Y}^{\vee}=\mathfrak{J}_{h, Y}+\mathfrak{J}_{h, Y}^{\vee} \mathfrak{J}_{h, Y}$ we easily deduce the identity $\mathfrak{J}_{h}^{\vee} h h K_{h}^{\vee}=\mathfrak{J}_{h}+\mathfrak{J}_{h}^{\vee} \mathfrak{J}_{h}$ we were looking for.

- There exists an $A$-linear isomorphism $\psi: \mathfrak{J}_{h}^{\vee} /\left(h \mathfrak{J}_{h}^{\vee}+h\left(\mathfrak{J}_{h}^{\vee}\right)^{2}\right) \cong \overline{\mathfrak{J}_{h}^{\vee}}-$ hence hereafter we shall identify $\overline{\mathfrak{J}_{h}^{\vee}}$ and $\mathfrak{J}_{h}^{\vee} /\left(h \mathfrak{J}_{h}^{\vee}+h\left(\mathfrak{J}_{h}^{\vee}\right)^{2}\right)$ via $\psi$ and $\psi^{-1}$.

Indeed, the natural projection map $K_{h}^{\vee} \longrightarrow \overline{K_{h}^{\vee}}:=K_{h}^{\vee} / h K_{h}^{\vee}$, whose kernel is $h K_{h}^{\vee}$, yields by restriction a similar map $\mathfrak{J}_{h}^{\vee} \longrightarrow \overline{\mathfrak{J}_{h}^{\vee}}:=\mathfrak{J}_{h}^{\vee} /\left(\mathfrak{J}_{h}^{\vee} \cap h K_{h}^{\vee}\right)$ whose kernel is $\left(\mathfrak{J}_{h}^{\vee} \cap h K_{h}^{\vee}\right)$. By the previous step, we have $\mathfrak{J}_{h}^{\vee} h h K_{h}^{\vee}=h \mathfrak{J}_{h}^{\vee}+h\left(\mathfrak{J}_{h}^{\vee}\right)^{2}$, whence we get an $A$-linear isomorphism.

- There exists an $A$-linear isomorphism $\sigma: \overline{\mathfrak{J}_{h}^{\vee}} \cong \mathfrak{J}_{h}^{\vee} /\left(h \mathfrak{J}_{h}^{\vee}+h\left(\mathfrak{J}_{h}^{\vee}\right)^{2}\right) \cong$ $\mathfrak{J} / \mathfrak{J}^{2}=: L^{*}$, where $\mathfrak{J} \equiv \mathfrak{J}_{J^{r}(L)}:=\operatorname{Ker}\left(\partial_{J^{r}(L)}\right)$, given by $\overline{h^{-1} y} \mapsto \sigma\left(\overline{h^{-1} y}\right):=\bar{y}$ $\bmod \mathfrak{J}^{2}$.

Indeed, there exists a natural projection map $\sigma^{\prime \prime}: \mathfrak{J}_{h} \longrightarrow \mathfrak{J}_{h} / h \mathfrak{J}_{h}=\mathfrak{J} \longrightarrow$ $\mathfrak{J} / \mathfrak{J}^{2}=: L^{*}$, whose kernel is $\left(h \mathfrak{J}_{h}+\mathfrak{J}_{h}^{2}\right)$. Then $\sigma^{\prime}: \mathfrak{J}_{h}^{\vee}:=h^{-1} \mathfrak{J}_{h} \longrightarrow \mathfrak{J} / \mathfrak{J}^{2}=$ : $L^{*}\left(h^{-1} y \mapsto \sigma^{\prime}\left(h^{-1} y\right):=\sigma^{\prime \prime}(y)\right)$ is a well defined $k$-linear map, whose kernel is $\left(\mathfrak{J}_{h}+h^{-1} \mathfrak{J}_{h}^{2}\right)=\left(h \mathfrak{J}_{h}^{\vee}+h\left(\mathfrak{J}_{h}^{\vee}\right)^{2}\right)$. Therefore $\sigma^{\prime}$ canonically induces a $k$-linear isomorphism $\sigma: \overline{\mathfrak{J}_{h}^{\vee}} \cong \mathfrak{J}_{h}^{\vee} /\left(h \mathfrak{J}_{h}^{\vee}+h\left(\mathfrak{J}_{h}^{\vee}\right)^{2}\right) \stackrel{\cong}{\longleftrightarrow} / \mathfrak{J}^{2}=: L^{*}$ given by $\overline{h^{-1} y} \mapsto$ $\sigma\left(\overline{h^{-1} y}\right):=\overline{\sigma^{\prime \prime}(y)}$; also, it is straightforward to check that this is $A$-linear too.

- We have $\overline{K_{h}^{\vee}} \in(\text { RQUEAd })_{A_{h}}$, namely $\overline{K_{h}^{\vee}} \cong V^{r}\left(L^{\prime}\right)$ for the Lie-Rinehart $A$-algebra $L^{\prime}:=\overline{\mathfrak{J}_{h}^{\vee}}$ (with the Lie-Rinehart structure mentioned above).

Indeed, what we proved so far shows that $L^{\prime}:=\overline{\mathfrak{J}_{h}^{\vee}}$ is a Lie-Rinehart subalgebra of $P^{r}\left(\overline{K_{h}^{\vee}}\right)$, which together with $A$ generates $\overline{K_{h}^{\vee}}$ (as an algebra) and is finite projective as an $A$-module (since it is isomorphic, as an $A$-module, to $L^{*}$, see above). Therefore, all conditions in Remark 3.3.5 are fulfilled, so it applies and gives $\overline{K_{h}^{\vee}} \cong V^{r}\left(L^{\prime}\right)$ for $L^{\prime}:=\overline{\mathfrak{J}_{h}^{\vee}}=P^{r}\left(\overline{K_{h}^{\vee}}\right)$.

- There exists on the Lie-Rinehart algebra $L^{\prime}$ a unique structure of LieRinehart bialgebra, canonically induced from the quantization $K_{h}^{\vee}$ of $V^{r}\left(L^{\prime}\right)$.

In fact, this is just a direct consequence of Theorem 4.1.6.

- The $A$-linear isomorphism $\sigma: \overline{\mathfrak{J}_{h}^{\vee}} \cong \mathfrak{J}_{h}^{\vee} /\left(h \mathfrak{J}_{h}^{\vee}+h\left(\mathfrak{J}_{h}^{\vee}\right)^{2}\right) \cong \mathfrak{J} / \mathfrak{J}^{2}=: L^{*}$ is actually an isomorphism of Lie-Rinehart bialgebras over $A$.

In order to prove this, we must show that $\sigma$ preserves the Lie bracket, the anchor map and the differential $\delta$ (cf. Definition 2.2.1) on either side.

For the Lie bracket, let $x, y \in \overline{\mathfrak{J}_{h}^{\vee}}$ : given $\chi, \eta \in \mathfrak{J}_{h}$ such that $x=h^{-1} \chi, y=$ $h^{-1} \eta$, we have

$$
[x, y]=h^{-2}(\chi \eta-\eta \chi) \bmod h K_{h}^{\vee}=h^{-2} h \zeta \bmod h K_{h}^{\vee}=h^{-1} \zeta \bmod h K_{h}^{\vee}
$$


for some $\zeta \in \mathfrak{J}_{h}$. But then also $\bar{\zeta}:=\zeta \bmod h K_{h}=:\{\bar{\chi}, \bar{\eta}\}-$ where $\bar{\alpha}:=$ $\alpha \bmod h K_{h}$ for all $\alpha \in K_{h}$ - by Theorem 4.1.12. Now the Poisson bracket of $K_{h} / h K_{h}$ restricted to $\mathfrak{J}_{h}$ pushes down to the Lie bracket of $\mathfrak{J}_{h} / \mathfrak{J}_{h}^{2}=: L^{*}$; thus setting $X:=\bar{\chi} \bmod \mathfrak{J}^{2}, Y:=\bar{\eta} \bmod \mathfrak{J}^{2}\left(\in \mathfrak{J} / \mathfrak{J}^{2}=: L^{*}\right)$, we have $[X, Y]=$ $\{\bar{\chi}, \bar{\eta}\} \bmod \mathfrak{J}^{2}=Z$. Now, by construction we have $X=\sigma(x), Y=\sigma(y)$, and the previous analysis eventually gives also $\sigma([x, y])=Z=[X, Y]=[\sigma(x), \sigma(y)]$.

For the anchor map, let $x \in \overline{\mathfrak{J}_{h}^{\vee}}, \chi \in \mathfrak{J}_{h}, X \in \mathfrak{J} / \mathfrak{J}^{2}=L^{*}$ as above, and take $a \in A$ and $a^{\prime} \in A_{h}$ such that $a^{\prime} \bmod h A_{h}=a$. Then direct computations give

$$
\begin{aligned}
\omega(x)(a) & =\partial_{h}\left(h^{-1} \chi s^{r}\left(a^{\prime}\right)-s^{r}\left(a^{\prime}\right) h^{-1} \chi\right) \bmod h A_{h} \\
& =\partial\left(h^{-1}\left(\chi s^{r}\left(a^{\prime}\right)-s^{r}\left(a^{\prime}\right) \chi\right) \bmod h K_{h}\right)=\omega(X)(a)
\end{aligned}
$$

which means $\omega(x)=\omega(X)=\omega(\sigma(x))$, that is $\sigma$ preserves the anchor, q.e.d.

Finally, in order to compare the two differentials on $\overline{\mathfrak{J}_{h}^{\vee}}$ and $L^{*}$, respectively denoted $\delta^{\prime}$ and $\delta^{\prime \prime}$, recall that in any Lie-Rinehart bialgebra $(\mathcal{L}, \mathcal{A})$ - in the present case $\left(L^{*}, A\right)$ - the differential $\delta_{\mathcal{L}}$ is related with the Lie bracket and the anchor map by the identities

$$
\begin{aligned}
\left\langle\mathrm{f}, \delta_{\mathcal{L}}(a)\right\rangle & =\omega_{\mathcal{L}^{*}}(\lambda)(a), \\
\left\langle\mathrm{f} \otimes \mu, \delta_{\mathcal{L}}(x)\right\rangle & =\omega_{\mathcal{L}^{*}}(\mathrm{f})(\langle\mathrm{m}, x\rangle)-\omega_{\mathcal{L}^{*}(\mathrm{~m})}(\langle\mathrm{f}, x\rangle)-\left\langle[\mathrm{f}, \mathrm{m}]_{\mathcal{L}^{*}}, x\right\rangle
\end{aligned}
$$

for all $x \in \mathcal{L}$, f, $\mathrm{m} \in \mathcal{L}^{*}, a \in A-$ see Remarks 2.2 .2 (b). We apply this to $(\mathcal{L}, \mathcal{A})=\left(L^{*}, A\right)$.

For the differential on $A$, we must prove that $\sigma\left(\delta^{\prime}(a)\right)=\delta^{\prime \prime}(a)$ for all $a \in$ $A$, which amounts to showing that $\left\langle\mathrm{f}, \sigma\left(\delta^{\prime}(a)\right)\right\rangle=\left\langle\mathrm{f}, \delta^{\prime \prime}(a)\right\rangle$ for all $a \in A$ and all $\mathrm{f} \in L$. For this comparison, recall that $V^{\ell}(L)_{h}:={ }_{\star} J^{r}(L)_{h} \in\left(\right.$ LQUEAd) ${ }_{A_{h}}$ is a quantization of $V^{\ell}(L)$, by Theorem 5.2.2 (a); moreover, the natural pairing between $V^{\ell}(L)_{h}$ and $J^{r}(L)_{h}$ (given by evaluation) is a right bialgebroid pairing. Now choose a lifting $a^{\prime} \in A_{h}$ of $a \in A$ and a lifting $f^{\prime} \in V^{\ell}(L)_{h}$ of $\mathrm{f} \in L$ : more precisely, we choose $f^{\prime} \in \operatorname{Ker}\left(\epsilon_{V^{\ell}(L)_{h}}\right)$. Then direct computation gives

$$
\begin{aligned}
\left\langle\mathrm{f}, \sigma\left(\delta^{\prime}(a)\right)\right\rangle & =h \cdot\left\langle f^{\prime}, \delta^{\prime}(a)\right\rangle \bmod h A_{h}=\left\langle f^{\prime}, s^{r}\left(a^{\prime}\right)-t^{r}\left(a^{\prime}\right)\right\rangle \bmod h A_{h} \\
& =\left\langle f^{\prime} s^{\ell}\left(a^{\prime}\right)-s^{\ell}\left(a^{\prime}\right) f^{\prime}, 1\right\rangle \bmod h A_{h} \\
& =\left\langle f^{\prime} s^{\ell}\left(a^{\prime}\right)-t^{\ell}\left(a^{\prime}\right) f^{\prime}, 1\right\rangle \bmod h A_{h} \\
& =\left\langle f^{\prime} s^{\ell}\left(a^{\prime}\right), 1\right\rangle \bmod h A_{h} \\
& =\epsilon_{V^{\ell}(L)}(\mathrm{f} a)=\omega_{L}(\mathrm{f})(a)=\left\langle\mathrm{f}, \delta^{\prime \prime}(a)\right\rangle
\end{aligned}
$$

(cf. $\S 3.2 .9$ for the last but one identity). This proves that $\sigma\left(\delta^{\prime}(a)\right)=\delta^{\prime \prime}(a)$ for all $a \in A$.

For the differential on $L^{*}$, consider $x:=\overline{\chi^{\vee}}=\overline{h^{-1} \chi} \in \overline{\mathfrak{J}_{h}^{\vee}}$, with $\chi \in \mathfrak{J}_{h}$; then we have $\sigma(x):=\bar{\chi} \bmod \mathfrak{J}^{2}=: X \in \mathfrak{J} / \mathfrak{J}^{2}=L^{*}$. Our goal is to prove that $(\sigma \otimes \sigma)\left(\delta^{\prime}(x)\right)=\delta^{\prime \prime}(\sigma(x))$. 
Write $\Delta(\chi)=\chi_{(1)} \otimes \chi_{(2)}$ as $\Delta(\chi)=\chi \otimes 1+1 \otimes \chi+\sum_{[\theta]} \chi_{[1]} \otimes \chi_{[2]}$; then we have $\Delta\left(\chi^{\vee}\right)=\chi^{\vee} \otimes 1+1 \otimes \chi^{\vee}+h \sum_{[\theta]} \chi_{[1]}^{\vee} \otimes \chi_{[2]}^{\vee}$ - where $\chi_{[i]}^{\vee}:=$ $h^{-1} \chi_{[i]}$, for $i \in\{1,2\}-$ so that $\delta^{\prime}(x):=-\sum_{[\theta]} x_{[1]} \otimes x_{[2]}+\sum_{[\theta]} x_{[2]} \otimes x_{[1]}$ with $x_{[i]}:=\overline{\chi_{[i]}^{\vee}}$ for $i \in\{1,2\}$. In all this, $\chi^{\vee}:=h^{-1} \chi$ is a lifting of $x \in L^{\prime}$ in $V^{r}\left(L^{\prime}\right)_{h}:=J^{r}(L)_{h}^{\vee}$, and $\chi$ is a lifting of $X:=\sigma(x)$ in $J^{r}(L)_{h}$; in addition, we can assume that $\partial_{h}(\chi)=0$. We adopt similar remarks, and notation, for $x_{[i]}, \chi_{[i]}$ and $X_{[i]}:=\sigma\left(x_{[i]}\right)$ with $i \in\{1,2\}$. Now for $\mathrm{f}, \mathrm{m} \in L$ and liftings $f^{\prime}, m^{\prime} \in V^{\ell}(L)_{h}$ of them, direct calculation yields

$$
\begin{aligned}
\left\langle\mathrm{f} \otimes \mathrm{m}, \delta^{\prime \prime}(\sigma(x))\right\rangle= & \left\langle\mathrm{f} \otimes \mathrm{m}, \delta^{\prime \prime}(X)\right\rangle \\
= & \omega_{L}^{\prime \prime}(\mathrm{f})(\langle\mathrm{m}, X\rangle)-\omega_{L}^{\prime \prime}(\mathrm{m})(\langle\mathrm{f}, X\rangle)-\left\langle[\mathrm{f}, \mathrm{m}]_{L}^{\prime \prime}, X\right\rangle \\
= & \epsilon_{V^{\ell}(L)}(\mathrm{f}\langle\mathrm{m}, X\rangle)-\epsilon_{V^{\ell}(L)}(\mathrm{m}\langle\mathrm{f}, X\rangle)-\langle\mathrm{fm}-\mathrm{mf}, X\rangle \\
= & \left(\left\langle f^{\prime} \cdot t^{\ell}\left(\left\langle m^{\prime}, \chi\right\rangle\right), 1\right\rangle-\left\langle m^{\prime} \cdot t^{\ell}\left(\left\langle f^{\prime}, \chi\right\rangle\right), 1\right\rangle\right. \\
& \left.\quad-\left\langle f^{\prime} m^{\prime}-m^{\prime} f^{\prime}, \chi\right\rangle\right) \bmod h A_{h} \\
= & \left(\left\langle f^{\prime} \cdot t^{\ell}\left(\left\langle m^{\prime}, \chi\right\rangle\right), 1\right\rangle-\left\langle m^{\prime} \cdot t^{\ell}\left(\left\langle f^{\prime}, \chi\right\rangle\right), 1\right\rangle\right. \\
& \quad-\left\langle f^{\prime} \cdot t^{\ell}\left(\left\langle f^{\prime}, \chi_{(2)}\right\rangle\right), \chi_{(1)}\right\rangle \\
& \left.\quad+\left\langle m^{\prime} \cdot t^{\ell}\left(\left\langle f^{\prime}, \chi_{(2)}\right\rangle\right), \chi_{(1)}\right\rangle\right) \bmod h A_{h} \\
= & \left(\left\langle m^{\prime} \cdot t^{\ell}\left(\left\langle f^{\prime}, \chi_{[2]}\right\rangle\right), \chi_{[1]}\right\rangle\right. \\
& \left.\quad-\left\langle f^{\prime} \cdot t^{\ell}\left(\left\langle m^{\prime}, \chi_{[2]}\right\rangle\right), \chi_{[1]}\right\rangle\right) \bmod h A_{h} \\
= & \left(\left\langle m^{\prime}, \chi_{[1]} s^{r}\left(\left\langle f^{\prime}, \chi_{[2]}\right\rangle\right)\right\rangle\right. \\
& \left.\quad-\left\langle f^{\prime}, \chi_{[1]} s^{r}\left(\left\langle m^{\prime}, \chi_{[2]}\right]\right)\right\rangle\right) \bmod h A_{h} \\
= & \left(\left\langle m^{\prime}, \chi_{[1]} t^{r}\left(\left\langle f^{\prime}, \chi_{[2]}\right\rangle\right)\right\rangle\right. \\
& \left.\quad-\left\langle f^{\prime}, \chi_{[1]} t^{r}\left(\left\langle m^{\prime}, \chi_{[2]}\right\rangle\right)\right\rangle\right) \bmod h A_{h} \\
= & \left(\left\langle m^{\prime}, \chi_{[1]}\right\rangle\left\langle f^{\prime}, \chi_{[2]}\right\rangle-\left\langle f^{\prime}, \chi_{[1]]}\right\rangle\left\langle m^{\prime}, \chi_{[2]}\right\rangle\right) \quad \bmod h A_{h} \\
= & \left\langle\mathrm{m}, \sigma\left(x_{[1]}\right)\right\rangle\left\langle\mathrm{f}, \sigma\left(x_{[2]}\right)\right\rangle-\left\langle\mathrm{f}, \sigma\left(x_{[1]}\right)\right\rangle\left\langle\mathrm{m}, \sigma\left(x_{[2]}\right)\right\rangle \\
= & \left\langle\mathrm{f} \otimes \mathrm{m},(\sigma \otimes \sigma)\left(\Delta^{[1]}(x)_{2,1}-\Delta^{[1]}(x)\right)\right\rangle \\
= & \left\langle\mathrm{f} \otimes \mathrm{m},(\sigma \otimes \sigma)\left(\delta^{\prime}(x)\right)\right\rangle
\end{aligned}
$$

Here above we used the fact that $s^{r}\left(\left\langle f^{\prime}, \chi_{[2]}\right\rangle\right)-t^{r}\left(\left\langle m^{\prime}, \chi_{[2]}\right\rangle\right)$ belongs to $\mathfrak{J}_{h}$, so that we have $\left.\chi_{[1]}\left(s^{r}\left(\left\langle f^{\prime}, \chi_{[2]}\right\rangle\right)\right)-t^{r}\left(\left\langle f^{\prime}, \chi_{[2]}\right\rangle\right)\right) \in \mathfrak{J}_{h}^{2}$ and $\left\langle m^{\prime}, \chi_{[1]}\left(s^{r}\left(\left\langle f^{\prime}, \chi_{[2]}\right\rangle\right)-\right.\right.$ $\left.\left.t^{r}\left(\left\langle f^{\prime}, \chi_{[2]}\right\rangle\right)\right)\right\rangle=0 \bmod h A_{h}$. Thus $\left\langle\mathrm{f} \otimes \mathrm{m}, \delta^{\prime \prime}(\sigma(x))\right\rangle=\left\langle\mathrm{f} \otimes \mathrm{m},(\sigma \otimes \sigma)\left(\delta^{\prime}(x)\right)\right\rangle$ for $\mathrm{f}, \mathrm{m} \in L$, so $\delta^{\prime \prime}(\sigma(x))=(\sigma \otimes \sigma)\left(\delta^{\prime}(x)\right)$.

In the end, all the above eventually completes the proof of claim (a.1). 
As to claim (a.2), let $\left(K_{h}, A_{h}, s_{K_{h}}^{r}, t_{K_{h}}^{r}, \Delta, \partial_{K_{h}}\right)$ and $\left(\Gamma_{h}, B_{h}, s_{\Gamma_{h}}^{r}, t_{\Gamma_{h}}^{r}, \Delta, \partial_{\Gamma_{h}}\right)$ be two RQFSAd's, and let $(f, \phi): K_{h} \longrightarrow \Gamma_{h}$ be a morphism between them in (RQFSAd). The very definition of morphism in (RQFSAd) imply at once that $\phi\left(s_{K_{h}}^{r}\left(A_{h}\right)\right) \subseteq s_{\Gamma_{h}}^{r}\left(B_{h}\right)-$ because $\phi \circ s_{K_{h}}^{r}=s_{\Gamma_{h}}^{r} \circ f-$ and $\phi\left(I_{K_{h}}\right) \subseteq I_{\Gamma_{h}}$ - because $\partial_{\Gamma_{h}} \circ \phi=\partial_{K_{h}}$ - hence also $\phi\left(h^{-1} I_{K_{h}}\right) \subseteq h^{-1} I_{\Gamma_{h}}$ for the natural $k((h))$-linear extension of $\phi: K_{h} \longrightarrow \Gamma_{h}$ to $\phi^{\times}:\left(K_{h}\right)_{F} \longrightarrow\left(\Gamma_{h}\right)_{F}$. By construction, this implies that $\phi^{\times}$defines by restriction a morphism $\phi^{\times}: K_{h}^{\times} \longrightarrow \Gamma_{h}^{\times}$, and this in turn extends by $h$-adic continuity to a well defined morphism $\phi^{\vee}: K_{h}^{\vee} \longrightarrow \Gamma_{h}^{\vee}$ in the category (RQUEAd).

(b) A direct proof of (b) can be given mimicking that of (a). Otherwise, it can be deduced from (a) (and, clearly, the rôles of the two results in this deduction can be reversed) as follows.

If $\Gamma_{h}:=J^{\ell}(L)_{h} \in(\text { LQFSAd })_{A_{h}}$, then $\left(\Gamma_{h}\right)_{\text {coop }}^{o p} \in(\mathrm{RQFSAd})_{A_{h}}$; thus by claim (a) we have that $\left(\left(\Gamma_{h}\right)_{\text {coop }}^{o p}\right)^{\vee} \in(\operatorname{RQUEAd})_{A_{h}}$. Now, by construction $\left(\left(\Gamma_{h}\right)_{\text {coop }}^{o p}\right)^{\vee}=$ $\left(\Gamma_{h}^{\vee}\right)_{\text {coop }}^{o p}$, hence we deduce that $\Gamma_{h}^{\vee} \in(\text { LQUEAd })_{A_{h}}$. All other aspects of the claim also follow from this argument.

6.2. The Drinfeld's functor(s) ()$^{\prime}={ }^{\prime}()$. We introduce now a second type of Drinfeld's functor, denoted $H \mapsto H^{\prime}$. Just like for the functor $H \mapsto H^{\vee}$, this also is inspired by the similar notion introduced for "quantum" Hopf algebras (see [12]); nevertheless, in this case we must be more careful, as we shall presently explain.

Let $H_{h}$ be a left (or a right) bialgebroid. If $s_{h}^{\ell}=t_{h}^{\ell}=: \iota_{h}^{\ell}$, then we can define $H^{\prime}$ as in the "classical" framework of quantum Hopf algebra deformations. Let us shortly recall it. Set $\delta_{n}=\left(i d_{H}-s^{\ell} \circ \epsilon\right)^{\otimes n} \circ \Delta^{n}$, where $\left(i d_{H}-s^{\ell} \circ \epsilon\right)^{\otimes n}$ is the projection of $H^{\otimes n}$ onto $\mathfrak{J}^{\otimes n}$ defined by the decomposition $H=\mathfrak{J} \oplus s_{\ell}(A)$, with $\mathfrak{J}:=\operatorname{Ker}(\epsilon)$ : then we define

$$
H^{\prime}:=\left\{a \in H \mid \delta_{n}(a) \in h^{n} H^{n} \quad \forall n \in \mathbb{N}\right\} \subseteq H .
$$

If instead $s^{\ell}$ and $t^{\ell}$ do not coincide, then the projection of $H^{\otimes n}$ onto $\mathfrak{J}^{\otimes n}$ is not defined, because the $\left(A_{h} \otimes A_{h}^{o p}\right)$-module $\mathfrak{J}_{h}$ does not have a complement in $H_{h}$. Therefore, as $s^{\ell}$ and $t^{\ell}$ do not necessarily coincide, we adopt the following definition:

Definition 6.2.1. As above, we use notation $\left(H_{h}\right)_{F}:=k((h)) \otimes_{k[[h]]} H_{h}$.

(a) If $H_{h} \in\left(\right.$ LQUEAd) $A_{h}$, we define

$$
H_{h}^{\prime}:=\left\{\eta \in\left(H_{h}\right)_{F} \mid\left\langle\eta,\left(H_{h}^{*}\right)^{\times}\right\rangle \in A_{h}\right\}, \quad{ }^{\prime} H_{h}:=\left\{\eta \in\left(H_{h}\right)_{F} \mid\left\langle\eta,\left(\left(H_{h}\right)_{*}\right)^{\times}\right\rangle \in A_{h}\right\}
$$

(b) If $H_{h} \in\left(\right.$ RQUEAd) $A_{h}$, we define

$$
H_{h}^{\prime}:=\left\{\eta \in\left(H_{h}\right)_{F} \mid\left\langle\eta,\left({ }^{*} H_{h}\right)^{\times}\right\rangle \in A_{h}\right\}, \quad{ }^{\prime} H_{h}:=\left\{\eta \in\left(H_{h}\right)_{F} \mid\left\langle\eta,\left({ }_{*}\left(H_{h}\right)\right)^{\times}\right\rangle \in A_{h}\right\}
$$


Proposition 6.2.2. Let $H_{h} \in(\mathrm{LQUEAd})_{A_{h}}$. Then

(a)

$$
\begin{gathered}
H_{h}^{\prime} \subseteq H_{h}, \quad{ }^{\prime} H_{h} \subseteq H_{h} \\
H_{h}^{\prime}={ }_{*}\left(\left(H_{h}^{*}\right)^{\times}\right)={ }_{*}\left(\left(H_{h}^{*}\right)^{\vee}\right), \quad{ }^{\prime} H_{h}={ }^{*}\left(\left(H_{h}\right)_{*}^{\times}\right)={ }^{*}\left(\left(H_{h}\right)_{*}{ }^{\vee}\right) \\
H_{h}^{\prime}=\left\{\lambda: H_{h}^{*} \rightarrow A_{h} \mid \lambda\left(u+u^{\prime}\right)=\lambda(u)+\lambda\left(u^{\prime}\right),\right. \\
\left.\lambda\left(u t^{r}(a)\right)=a \lambda(u), \lambda\left(I_{H_{h}^{*}}^{n}\right) \subseteq h^{n} A_{h} \forall n\right\} \\
{ }^{\prime} H_{h}=\left\{\lambda: H_{h *} \rightarrow A_{h} \mid \lambda\left(u+u^{\prime}\right)=\lambda(u)+\lambda\left(u^{\prime}\right),\right. \\
\left.\lambda\left(u s^{r}(a)\right)=\lambda(u) a, \lambda\left(I_{H_{h *}}^{n}\right) \subseteq h^{n} A_{h} \forall n\right\}
\end{gathered}
$$

(b) The analogous results hold if $H_{h} \in(\mathrm{RQUEAd}){ }_{A_{h}}$.

Proof. The proof is the same as in [12], hence we do not need to reproduce it.

Remark 6.2.3. If $H_{h} \in(\text { LQUEAd })_{A_{h}}$, then $\left(\left(H_{h}\right)_{\text {coop }}^{o p}\right)^{\prime}=\left({ }^{\prime} H_{h}\right)_{\text {coop }}^{o p}$. This follows from the following three remarks:

- if $U$ is any left bialgebroid, then $\left(U_{*}\right)_{\text {coop }}^{o p} \cong{ }^{*}\left(U_{\text {coop }}^{o p}\right)$ as left bialgebroids;

- if $W$ is any right bialgebroid, then $\left({ }^{*} W\right)_{\text {coop }}^{o p} \cong\left(W_{\text {coop }}^{o p}\right)_{*}$ as right bialgebroids;

- the functor ()$^{\vee}$ commutes with the functor ()$_{\text {coop }}^{o p}$.

Similarly, one has ' $\left(\left(H_{h}\right)_{\text {coop }}^{o p}\right)=\left(H_{h}^{\prime}\right)_{\text {coop }}^{o p}$. Finally, in the same way one finds also the parallel identities $\left(\left(H_{h}\right)_{\text {coop }}^{o p}\right)^{\prime}=\left({ }^{\prime} H_{h}\right)_{\text {coop }}^{o p}$ and ${ }^{\prime}\left(\left(H_{h}\right)_{\text {coop }}^{o p}\right)=\left(H_{h}^{\prime}\right)_{\text {coop }}^{o p}$ for every $H_{h} \in(\text { RQUEAd })_{A_{h}}$.

6.2.4. Explicit description of ' $H_{h}$. For a given $H_{h} \in\left(\right.$ LQUEAd) $A_{h}$, we can describe ' $H_{h}$ quite explicitly. Write $H_{h}=\mathfrak{J}_{h} \oplus s_{\ell}\left(A_{h}\right)$, and let $\pi_{s}$ be the projection of $H_{h}$ onto $\mathfrak{J}_{h}$ : this is not a morphism of $\left(A_{h} \otimes A_{h}^{o p}\right)$-modules. We need another lemma, whose proof is left to the reader:

Lemma 6.2.5. For any $u \in H_{h}$ and $a \in A_{h}$, one has $\pi_{s}\left(s^{\ell}(a) u\right)=s^{\ell}(a) \pi_{s}(u)$.

If in addition $t^{\ell}(a)-s^{\ell}(a)=h j$ for some $j \in \mathfrak{J}_{h}$, then $\pi_{s}\left(t^{\ell}(a) u\right)=$ $s^{\ell}(a) \pi_{s}(u)+h \pi_{s}(j u)$.

The operator $\pi_{s}^{\otimes n}$ is not defined on $H_{h} \otimes_{A_{h}} H_{h} \otimes A_{h} \cdots \otimes_{A_{h}} H_{h}$. If $u_{1} \otimes \cdots \otimes u_{n} \in$ $H_{h} \otimes_{A_{h}} H_{h} \otimes_{A_{h}} \cdots \otimes_{A_{h}} H_{h}$, then $\pi_{s}\left(u_{1}\right) \otimes \cdots \otimes \pi_{s}\left(u_{n}\right)$ depends on the way of writing of $u_{1} \otimes \cdots \otimes u_{n}$. We will say that the component of $\sum u_{1} \otimes \cdots \otimes u_{n}$ in $\mathfrak{J}_{h}^{\otimes n}$ is defined up to $h^{n} \mathfrak{J}_{h}^{\otimes n}$ if $\sum u_{1} \otimes \cdots \otimes u_{n}=\sum v_{1} \otimes \cdots \otimes v_{n}$ implies $\sum \pi_{s}\left(u_{1}\right) \otimes \cdots \otimes \pi_{s}\left(u_{n}\right)-\sum \pi_{s}\left(v_{1}\right) \otimes \cdots \otimes \pi_{s}\left(v_{n}\right) \in h^{n} \mathfrak{J}_{h}^{\otimes n}$.

Lemma 6.2.6. Let $u \in H_{h}$ and $n \in \mathbb{N}_{+}$. If the component of $\Delta^{n}(u)$ in $\mathfrak{J}_{h}^{\otimes n}$ is defined up to $h^{n} \mathfrak{J}_{h}^{\otimes n}$ and belongs to $h^{n} \mathfrak{J}_{h}^{\otimes n}$, then the component of $\Delta^{n+1}(u)$ is defined up to $h^{n+1} \mathfrak{J}_{h}^{\otimes(n+1)}$ - hence it makes sense to say that it belongs to $h^{n+1} \mathfrak{J}_{h}^{\otimes(n+1)}$. 
Proof. If the component of $\Delta^{n}(u)$ in $\mathfrak{J}_{h}^{\otimes n}$ belongs to $h^{n} \mathfrak{J}_{h}^{\otimes n}$, then $\Delta^{n}(u)$ can be written as

$$
\Delta^{n}(u)=\sum h^{n} \phi_{1} \otimes \cdots \otimes \phi_{n}+\text { other terms }
$$

where all the $\phi_{i}$ 's are in $\mathfrak{J}_{h}$ and "other terms" stands for a sum of homogeneous tensors containing (as tensor factors) elements of $s_{\ell}\left(A_{h}\right)$ which do not occur in the computation of the component of $\Delta^{n+1}(u)$ in $\mathfrak{J}_{h}^{n+1}$. Assume that $\Delta^{n+1}(u)$ can be written, for some $a \in A$, as

$$
\begin{aligned}
\Delta^{n+1}(u) & =\sum h^{n} \chi_{1} \otimes \cdots \otimes t^{\ell}(a) \chi_{i} \otimes \chi_{i+1} \otimes \cdots \otimes \chi_{n+1}+\text { other terms } \\
\text { or } \quad \Delta^{n+1}(u) & =\sum h^{n} \chi_{1} \otimes \cdots \otimes \chi_{i} \otimes s^{\ell}(a) \chi_{i+1} \otimes \cdots \otimes \chi_{n+1}+\text { other terms }
\end{aligned}
$$

and let us compute $\pi_{s}^{\otimes(n+1)}\left(\Delta^{n+1}(u)\right)$ in both cases.

In the second case, $\pi_{s}^{\otimes(n+1)}\left(\Delta^{n+1}(u)\right)$ can be written as

$\pi_{s}^{\otimes(n+1)}\left(\Delta^{n+1}(u)\right)=\sum h^{n} \pi_{s}\left(\chi_{1}\right) \otimes \cdots \otimes \pi_{s}\left(\chi_{i}\right) \otimes s_{\ell}(a) \pi_{s}\left(\chi_{i+1}\right) \otimes \cdots \otimes \pi_{s}\left(\chi_{n+1}\right)$

In the first case, if we write $t^{\ell}(a)-s^{\ell}(a)=h j$ (with $j \in \mathfrak{J}_{h}$ ) and use the previous lemma, we get

$$
\begin{aligned}
& \pi_{s}^{\otimes(n+1)}\left(\Delta^{n+1}(u)\right)=\sum h^{n} \pi_{s}\left(\chi_{1}\right) \otimes \cdots \otimes t^{\ell}(a) \pi_{s}\left(\chi_{i}\right) \otimes \pi_{s}\left(\chi_{i+1}\right) \otimes \cdots \otimes \pi_{s}\left(\chi_{n+1}\right) \\
& +\sum h^{n} \pi_{s}\left(\chi_{1}\right) \otimes \cdots \otimes \pi_{S}\left(\chi_{i-1}\right) \otimes h\left(-j \pi_{s}\left(\chi_{i}\right)+\pi_{s}\left(j \chi_{i}\right)\right) \otimes \pi_{s}\left(\chi_{i+1}\right) \cdots \otimes \pi_{s}\left(\chi_{n+1}\right)
\end{aligned}
$$

Taking the difference between the two computations we find

$h^{n} \pi_{s}\left(\chi_{1}\right) \otimes \cdots \otimes \pi_{s}\left(\chi_{i-1}\right) \otimes h\left(-j \pi_{s}\left(\chi_{i}\right)+\pi_{s}\left(j \chi_{i}\right)\right) \otimes \pi_{s}\left(\chi_{i+1}\right) \cdots \otimes \pi_{s}\left(\chi_{n+1}\right)$

which does belong to $h^{n+1} \mathfrak{J}^{\otimes(n+1)}$, q.e.d.

Notation. If the component of $\Delta^{n}(u)$ in $\mathfrak{J}_{h}^{\otimes n}$ is defined up to $h^{n} \mathfrak{J}^{\otimes n}$, we shall write it as $\delta_{s}^{n}(u)$. Then the condition $\delta_{s}^{n}(u) \in h^{n} \mathfrak{J}^{\otimes n}$ perfectly makes sense. Hereafter we shall write $\delta_{s}^{n}(u) \in h^{n} \mathfrak{J}^{\otimes n}$ to mean that $\delta_{s}^{n}(u)$ is well defined - i.e., the component of $\Delta^{n}(u)$ in $\mathfrak{J}^{\otimes n}$ is well defined - up to $h^{n} \mathfrak{J}^{\otimes n}$ and it belongs to $h^{n} \mathfrak{J}^{\otimes n}$. For the rest of the discussion, we introduce the notation

$$
\delta_{s}\left(H_{h}\right):=\left\{u \in H_{h} \mid \delta_{s}^{n}(u) \in h^{n} \mathfrak{J}_{h}^{\otimes n} \forall n \in \mathbb{N}_{+}\right\}
$$

We need again a couple of technical results:

Proposition 6.2.7. Let $u \in \delta_{s}\left(H_{h}\right)$. Then $\Delta(u)$ can be written as

$$
\Delta(u)=u \otimes 1+\sum u_{(1)}^{\prime} \otimes u_{(2)}^{\prime} \text { with } u_{(1)}^{\prime} \in \delta_{S}\left(H_{h}\right) \text { and } u_{(2)}^{\prime} \in h \mathfrak{J}_{h}
$$


Proof. First case: $L$ is a finite free as an $A$-module.

Let $\left\{\bar{e}_{1}, \bar{e}_{2}, \ldots, \bar{e}_{n}\right\}$ be a basis of the $A$-module $L$ : we lift each $\bar{e}_{i}$ to an element $e_{i} \in H_{h}$ such that $\epsilon\left(e_{i}\right)=0$. Let $u \in \delta^{s}\left(H_{h}\right)$. We write $\Delta(u)$ as

$$
\Delta(u)=u^{\prime} \otimes 1+\sum_{\underline{\alpha} \in \mathbb{N}^{n} \backslash\{\underline{0}\}} u_{\underline{\alpha}} \otimes e^{\underline{\alpha}} \quad \text { with } \lim _{|\underline{\alpha}| \rightarrow+\infty}\left\|u_{\underline{\alpha}}\right\|=0 .
$$

for suitable $u^{\prime}, u_{\underline{\alpha}} \in H_{h}$. The relation $m_{H_{h}}\left(\left(s^{\ell} \circ \epsilon\right) \otimes i d\right)(\Delta(u))=u$ gives $u^{\prime}=u$. Thus we have

$$
\Delta(u)=u \otimes 1+\sum_{\underline{\alpha} \in \mathbb{N}^{n} \backslash\{\underline{0}\}} u_{\underline{\alpha}} \otimes e^{\underline{\alpha}}
$$

The relation $m_{H_{h}}\left(\left(s^{\ell} \circ \epsilon\right) \otimes i d\right)(\Delta(u))=u$ yields the identity

$$
\sum_{\underline{\alpha} \in \mathbb{N}^{n} \backslash\{\underline{0}\}} s^{\ell}\left(\epsilon\left(u_{\underline{\alpha}}\right)\right) e^{\underline{\alpha}}=u-s^{\ell}(\epsilon(u))
$$

As $u \in \delta_{S}\left(H_{h}\right)$, one has $u-s^{\ell}(\epsilon(u)) \in h \mathfrak{J}_{h}$, which implies that $s^{\ell}\left(\epsilon\left(u_{\underline{\alpha}}\right)\right) \in h H_{h}$; hence $\overline{s^{\ell}\left(\epsilon\left(u_{\alpha}\right)\right)}=\overline{s_{\ell}}\left(\overline{\epsilon\left(u_{\underline{\alpha}}\right)}\right)=0 \in H_{h} / h H_{h}$. As $\overline{s_{\ell}}$ is injective, we get $\overline{\epsilon\left(u_{\alpha}\right)}=0$, i.e. $\epsilon\left(u_{\alpha}\right) \in h A_{h}$.

If $n>1$, one has

$$
\delta_{s}^{n}(u)=\sum_{\underline{\alpha} \in \mathbb{N}^{n}} \delta_{s}^{n-1}\left(u_{\underline{\alpha}}\right) \otimes e^{\underline{\alpha}} \in h^{n} \mathfrak{J}^{\otimes n}
$$

which implies $\delta_{s}^{n-1}\left(u_{\underline{\alpha}}\right) \in h^{n} \mathfrak{J}^{\otimes(n-1)}$ and $u_{\underline{\alpha}} \in \delta_{s}\left(H_{h}\right)$. Let $\widetilde{u}_{\underline{\alpha}}=\pi_{s}\left(u_{\underline{\alpha}}\right)=$ $u_{\underline{\alpha}}-s^{\ell}\left(\epsilon\left(u_{\underline{\alpha}}\right)\right)$. For all $n \geq 1$, one has $\left.\delta_{s}^{n} \widetilde{u}_{\underline{\alpha}}\right)=\delta_{s}^{n}\left(u_{\underline{\alpha}}\right) \in h^{n+1} \mathfrak{J}^{n}$. In particular for $n=1$ we get $\widetilde{u}_{\alpha}=h w_{\alpha}$ for some $w_{\alpha} \in \delta_{s}\left(H_{h}\right)$. The element $u_{\underline{\alpha}}$ can be written as $u_{\underline{\alpha}}=h\left(w_{\underline{\alpha}}+s^{\underline{\ell}}\left(h^{-1} \epsilon\left(u_{\underline{\alpha}}\right)\right)\right) \in h \delta_{s}\left(H_{h}\right)$.

Second case: $L$ is finite projective as an $A$-module.

Just as in Subsection 4.2, we fix a finite projective $A$-module $Q$ such that $L \oplus Q=F$ is a finite free $A$-module. We fix an $A$-basis $B:=\left\{e_{1}, \ldots, e_{n}\right\}$ of $F$ : then we call $Y$ the $k$-span of $B$, so that we can write $F=A \otimes_{k} Y$. Moreover, we construct the (infinite dimensional) Lie-Rinehart algebra $L_{Q}=L \oplus\left(A \otimes_{k} Z\right)$, with $Z=Y \oplus Y \oplus Y \oplus \cdots$, which has a good basis $\left\{e_{i}\right\}_{i \in T:=\mathbb{N} \times\{1, \ldots, n\}}$ defined by $B$. And as in \$4.2.1, we can define $H_{h, Y}$ and $\delta_{s}\left(H_{h, Y}\right)$. Now given $u \in \delta_{s}\left(H_{h, Y}\right)$, we can write $\Delta(u)$ as follows:

$$
\Delta(u)=u \otimes 1+\sum_{\underline{\alpha} \in T^{(\mathbb{N})} \backslash\{\underline{0}\}} u_{\underline{\alpha}} \otimes e^{\underline{\alpha}} \quad \text { with } \quad \lim _{|\underline{\alpha}|+\varpi(\alpha) \rightarrow+\infty}\left\|u_{\underline{\alpha}}\right\|=0
$$

Then the same reasoning as above shows that the proposition is true for $H_{h, Y}$ in the rôle of $H_{h}$.

Recall (cf. §4.2.1) that $H_{h, Y}=H_{h} \oplus\left(H_{h} \widehat{\otimes}_{k} S(Z)^{+}\right)$where $H_{h} \widehat{\otimes}_{k} S(Z)^{+}$ is the $h$-adic completion of $H_{h} \otimes_{k} S(Z)^{+}$, with $Z=Y \oplus Y \oplus Y \oplus \cdots$; the natural projection $\pi_{Y}: H_{h, Y} \longrightarrow H_{h}$ is then a morpism of left bialgebroids. Moreover, if $\mathfrak{J}_{h, Y}$ is the kernel of the counit of $H_{h, Y}$, we have $\mathfrak{J}_{h, Y}=\mathfrak{J}_{h} \oplus$ $\left(H_{h} \widehat{\otimes}_{k} S(Z)^{+}\right)$. Now it is easy to see that, if $v \in \delta_{s}\left(H_{h, Y}\right)$, then $\pi_{Y}(v) \in \delta_{s}\left(H_{h}\right)$. 
Now let $u \in \delta_{s}\left(H_{h}\right)$. By the result for $H_{h, Y}$, we know that $\Delta(u)$ can be written as

$$
\Delta(u)=u \otimes 1+\sum u_{(1)}^{\prime} \otimes u_{(2)}^{\prime} \quad \text { with } \quad u_{(1)}^{\prime} \in \delta_{S}\left(H_{h, Y}\right) \quad \text { and } \quad u_{(2)}^{\prime} \in h \mathfrak{J}_{h, Y}
$$

As $\pi_{Y}(u)=u$, applying $\pi_{Y} \otimes \pi_{Y}$ to the previous identity we get

$$
\Delta(u)=u \otimes 1+\sum \pi_{Y}\left(u_{(1)}^{\prime}\right) \otimes \pi_{Y}\left(u_{(2)}^{\prime}\right)
$$

with $\pi_{Y}\left(u_{(1)}^{\prime}\right) \in \pi_{Y}\left(\delta_{S}\left(H_{h, Y}\right)\right)=\delta_{S}\left(H_{h}\right)$ and $\left.\pi_{Y}\left(u_{(2)}\right) \in h \pi_{Y}\left(\mathfrak{J}_{h, Y}\right)\right)=h \mathfrak{J}_{h}$.

Lemma 6.2.8. $s^{\ell}\left(A_{h}\right) \cdot \delta_{s}\left(H_{h}\right) \subseteq \delta_{s}\left(H_{h}\right)$ and $t^{\ell}\left(A_{h}\right) \cdot \delta_{s}\left(H_{h}\right) \subseteq \delta_{s}\left(H_{h}\right)$.

Proof. Let $u \in \delta_{s}\left(H_{h}\right)$ and $a \in A_{h}$. The properties $s^{\ell}(a) u \in \delta_{s}\left(H_{h}\right)$ follows from the following properties: $\pi_{s}\left(s^{\ell}(a) u\right)=s^{\ell}(a) \pi_{s}(u)$ and $\Delta^{n}\left(s^{\ell}(a)\right)=$ $s^{\ell}(a) \otimes 1 \otimes \cdots \otimes 1$. Let us now show that $\delta_{s}^{n}\left(t^{\ell}(a) u\right) \in h^{n} \mathfrak{J}^{\otimes n}$ for all $n \in \mathbb{N}$. Write $t^{\ell}(a)-s^{\ell}(a)=h j$ with $j \in \mathfrak{J}_{h}$.

For $n=1$, by Lemma 6.2.5 we have $\pi_{s}\left(t^{\ell}(a) u\right)=s^{\ell}(a) \pi_{s}(u)+h \pi_{s}(j u) \in h \mathfrak{J}$.

For $n>1$, let us show that $\delta_{s}^{n}\left(t^{\ell}(a)\right) \in h^{n} \mathfrak{J}^{\otimes n}$. Set $\Delta(u)=u \otimes 1+u_{(1)}^{\prime} \otimes u_{(2)}^{\prime}$ with $u_{(1)}^{\prime} \in \delta_{s}\left(H_{h}\right), u_{(2)}^{\prime} \in h \mathfrak{J}_{h}$ (cf. Proposition 6.2.7). Then $\Delta\left(t^{\ell}(a) u\right)=u \otimes$ $t^{\ell}(a)+u_{(1)}^{\prime} \otimes t^{\ell}(a) u_{(2)}^{\prime}$, hence $\delta_{s}^{n}\left(t^{\ell}(a) u\right)=\delta_{s}^{n-1}(u) \otimes \pi_{s}\left(t^{\ell}(a)\right)+\delta_{s}^{n-1}\left(u_{(1)}^{\prime}\right) \otimes$ $\pi_{s}\left(t^{\ell}(a) u_{(2)}^{\prime}\right)$, thus $\delta_{s}^{n}\left(t^{\ell}(a) u\right) \in h^{n} \mathfrak{J}^{\otimes n}$.

We are now ready for the first key result of this subsection:

Theorem 6.2.9. With assumptions and notation as above, we have

$$
{ }^{\prime} H_{h}=\left\{u \in H_{h} \mid \delta_{s}^{n}(u) \in h^{n} \mathfrak{J}_{h}^{\otimes n} \forall n \in \mathbb{N}_{+}\right\}=: \delta_{s}\left(H_{h}\right)
$$

Proof. To begin with, we show that $\delta_{s}\left(H_{h}\right) \subseteq{ }^{\prime} H_{h}$. To this end, we prove that for any $u \in \delta_{s}(H)$ we have $\left\langle u, I_{\left(H_{h}\right)_{*}}^{n}\right\rangle \subseteq h^{n} A_{h}$ for all $n \in \mathbb{N}_{+}$, using induction on $n$.

Take $n=1$. As $u \in \delta_{s}(H)$, note that $\delta^{1}(u) \in h \mathfrak{J}_{h}$ implies $u=h j+s^{\ell}(\epsilon(u))$ with $j \in \mathfrak{J}_{h}$. Then one has

$$
\left\langle u, I_{H_{h *}}\right\rangle=h\left\langle j, I_{H_{h *}}\right\rangle+\epsilon(u)\left\langle 1, I_{H_{h *}}\right\rangle \in h A_{h}
$$

Now assume $n>1$. For our $u \in \delta_{s}(H)$, set $\Delta(u)=u \otimes 1+u_{(1)}^{\prime} \otimes u_{(2)}^{\prime}$ with $u_{(1)}^{\prime} \in \delta_{s}\left(H_{h}\right)$ and $u_{(2)}^{\prime} \in h \mathfrak{J}_{h}$ as in Proposition 6.2.7. Let $\alpha \in I_{H_{h *}}^{n}$ be of the form $\alpha=\alpha_{1} \alpha_{2}$ with $\alpha_{1} \in I_{H_{h *}}$ and $\alpha_{2} \in I_{H_{h *}}^{n-1}$ : then, as the pairing $\langle$,$\rangle between H_{h}$ and $H_{h *}$ is a left bialgebroid pairing, we have

$$
\left\langle u, \alpha_{1} \alpha_{2}\right\rangle=\left\langle t^{\ell}\left(\left\langle u_{(2)}^{\prime}, \alpha_{1}\right\rangle\right) u_{(1)}^{\prime}, \alpha_{2}\right\rangle+\left\langle t^{\ell}\left(\left\langle 1, \alpha_{1}\right\rangle\right) u, \alpha_{2}\right\rangle \in h^{n} A_{h}
$$

by the induction hypothesis and the case $n=1$ (also using the two previous lemmas).

Conversely, let us now show that ' $H_{h} \subseteq \delta_{s}\left(H_{h}\right)$. To this end, we prove (by induction on $n$ ) that for any $u \in{ }^{\prime} H_{h}$ one has $\delta_{s}^{n}(u) \in h^{n} \mathfrak{J}_{h}^{\otimes n}$ for all $n \in \mathbb{N}$. 
For $n=1$. As $u \in{ }^{\prime} H_{h}$ we have $\left\langle u, I_{H_{h *}}\right\rangle \subseteq h A_{h}$; on the other hand, $\delta_{s}^{1}(u)=$ $u-s^{\ell}(\epsilon(u))$ by definition. Then we have $\left\langle\delta_{s}^{1}(u), \lambda\right\rangle \in h A_{h}$, if $\lambda \in I_{H_{h *}}$ because

$$
\begin{aligned}
\left\langle u-s^{\ell}(\epsilon(u)), \lambda\right\rangle=\langle u, \lambda\rangle-\left\langle s^{\ell}(\epsilon(u)), \lambda\right\rangle & =\langle u, \lambda\rangle-\epsilon(u)\langle 1, \lambda\rangle \\
& =\langle u, \lambda\rangle-\epsilon(u) \partial(\lambda) \in h A_{h}
\end{aligned}
$$

On the other hand, clearly $\delta_{s}^{1}(u)=u-s^{\ell}(\epsilon(u)) \in \mathfrak{J}_{h}$, hence $\delta_{s}^{1}(u) \in \mathfrak{J}_{h} \cap h H_{h}=h \mathfrak{J}_{h}$.

Let now $n>1$, and assume by induction that $\delta_{s}^{n-1}\left(u^{\prime}\right) \in h^{n-1} \mathfrak{J}^{\otimes(n-1)}$ for all $u^{\prime} \in{ }^{\prime} H_{h}$. For our $u \in{ }^{\prime} H_{h}$, write $\Delta(u)=u_{(1)} \otimes u_{(2)}$ with $u_{(1)}, u_{(2)} \in{ }^{\prime} H$. As $\Delta^{n}(u)=\Delta^{n-1}\left(u_{(1)}\right) \otimes u_{(2)}$, we get $\delta_{s}^{n}(u)=\delta_{s}^{n-1}\left(u_{(1)}\right) \otimes \delta_{s}^{1}\left(u_{(2)}\right) \in h^{n} \mathfrak{J}^{\otimes n}$ by the induction hypothesis and the case $n=1$.

6.2.10. Explicit description of $H_{h}^{\prime}$. We shall now give an explicit description of $H_{h}^{\prime}$ : this will be entirely similar to that for ' $H_{h}$, thus we shall only outline the main steps, without dwelling into details - which can be easily filled in by the reader.

Write $H_{h}=\mathfrak{J}_{h} \oplus t_{\ell}\left(A_{h}\right)$, and let $\pi_{t}$ be the projection of $H_{h}$ onto $\mathfrak{J}_{h}$ : once again, this is not a morphism of $\left(A_{h} \otimes A_{h}^{o p}\right)$-modules. The operator $\pi_{t}^{\otimes n}$ is not defined on $H_{h} \otimes_{A_{h}} H_{h} \otimes_{A_{h}} \cdots \otimes_{A_{h}} H_{h}$ : indeed, if $u_{1} \otimes \cdots \otimes u_{n} \in H_{h} \otimes_{A_{h}} H_{h} \otimes_{A_{h}} \cdots \otimes_{A_{h}} H_{h}$, then $\pi_{t}\left(u_{1}\right) \otimes \cdots \otimes \pi_{t}\left(u_{n}\right)$ depends on the way of writing $u_{1} \otimes \cdots \otimes u_{n}$. We say that the component of $\sum u_{1} \otimes \cdots \otimes u_{n}$ in $\mathfrak{J}_{h}^{\otimes n}$ is defined up to $h^{n} \mathfrak{J}_{h}^{\otimes n}$ if $\sum u_{1} \otimes \cdots \otimes u_{n}=$ $\sum v_{1} \otimes \cdots \otimes v_{n}$ yields $\sum \pi_{t}\left(u_{1}\right) \otimes \cdots \otimes \pi_{t}\left(u_{n}\right)-\sum \pi_{t}\left(v_{1}\right) \otimes \cdots \otimes \pi_{t}\left(v_{n}\right) \in h^{n} \mathfrak{J}_{h}^{\otimes n}$.

The following lemma is the parallel of Lemma 6.2.6, with similar proof. Note that the statement is formally the same, but actually the "components" to which one refers in the two claims are defined with respect to different projectors - namely $\pi_{s}^{\otimes n}$ or $\pi_{t}^{\otimes n}-$ in the two cases.

Lemma 6.2.11. Let $u \in H_{h}$. If the component of $\Delta^{n}(u)$ in $\mathfrak{J}_{h}^{\otimes n}$ is defined up to $h^{n} \mathfrak{J}_{h}^{\otimes n}$ and belongs to $h^{n} \mathfrak{J}_{h}^{\otimes n}$, then the component of $\Delta^{n+1}(u)$ is defined up to $h^{n+1} \mathfrak{J}_{h}^{\otimes(n+1)}$ - hence it makes sense to say that it belongs to $h^{n+1} \mathfrak{J}_{h}^{\otimes(n+1)}$.

Notation. If the component of $\Delta^{n}(u)$ in $\mathfrak{J}_{h}^{\otimes n}$ is defined up to $h^{n} \mathfrak{J}^{\otimes n}$ (in the above sense), we shall write it as $\delta_{t}^{n}(u)$. Then the condition $\delta_{t}^{n}(u) \in h^{n} \mathfrak{J}^{\otimes n}$ perfectly makes sense. Thus we shall write $\delta_{t}^{n}(u) \in h^{n} \mathfrak{J}^{\otimes n}$ to mean that $\delta_{t}^{n}(u)$ is well defined (i.e., the component of $\Delta^{n}(u)$ in $\mathfrak{J}^{\otimes n}$, in the above sense, is well defined) up to $h^{n} \mathfrak{J}^{\otimes n}$ and it belongs to $h^{n} \mathfrak{J}^{\otimes n}$. Also, we set

$$
\delta_{t}\left(H_{h}\right):=\left\{u \in H_{h} \mid \delta_{t}^{n}(u) \in h^{n} \mathfrak{J}_{h}^{\otimes n} \forall n \in \mathbb{N}_{+}\right\}
$$

Arguing as for ' $H_{h}$, we can then prove the following, analogous characterization of $H_{h}^{\prime}$ :

Theorem 6.2.12. With assumptions and notation as above, we have

$$
H_{h}^{\prime}=\left\{u \in H_{h} \mid \delta_{t}^{n}(u) \in h^{n} \mathfrak{J}_{h}^{\otimes n} \forall n \in \mathbb{N}_{+}\right\}=: \delta_{t}\left(H_{h}\right)
$$


Remark 6.2.13. The study of ' $H_{h}$ and $H_{h}^{\prime}$ we have done for LQUEAd holds for RQUEAd as well. One can check it directly (via the same arguments) or, by deducing the results for RQUEAd's from those for LQUEAd's in force of the general identities $\left(H_{h}^{\prime}\right)_{\text {coop }}^{o p}={ }^{\prime}\left(\left(H_{h}\right)_{\text {coop }}^{o p}\right)$.

Thanks to the characterizations in Theorem 6.2.9 and Theorem 6.2.12 we can eventually prove the following remarkable result:

Theorem 6.2.14. Let $H_{h}$ be a LQUEAd or a RQUEAd. Then $H_{h}^{\prime}={ }^{\prime} H_{h}$.

Proof. We begin with $H_{h}$ being an LQUEAd. We show, that for any $u \in \delta_{s}\left(H_{h}\right)$ we have $\delta_{t}^{n}(u) \in h^{n} \mathfrak{J}^{\otimes n}$ for all $n \in \mathbb{N}$, by induction on $n$.

For $n=1$, one has

$$
\delta_{t}^{1}(u)=u-t_{l}(\epsilon(u))=u-s_{\ell}(\epsilon(u))+s_{\ell}(\epsilon(u))-t_{\ell}(\epsilon(u))
$$

As $s^{\ell}-t^{\ell}=0 \bmod h$, one has $s^{\ell}(\epsilon(u))-t^{\ell}(\epsilon(u)) \in h A_{h}$. Moreover, we have also $\epsilon\left(s^{\ell}(\epsilon(u))-t^{\ell}(\epsilon(u))\right)=0$, so that $s^{\ell}(\epsilon(u))-t^{\ell}(\epsilon(u))$ belongs to $\mathfrak{J}_{h} \cap h A_{h}=h \mathfrak{J}_{h}$. Thus $\delta_{t}^{1}(u) \in h \mathfrak{J}_{h}$, q.e.d.

For $n>1$, let us write $\Delta(u)=u \otimes 1+u_{(1)}^{\prime} \otimes u_{(2)}^{\prime}$ with $u_{(1)}^{\prime} \in \delta_{s}\left(H_{h}\right)$ and $u_{(2)}^{\prime} \in h \mathfrak{J}_{h}$ as in Proposition 6.2.7. Then one has $\delta_{t}^{n}(u)=\delta_{t}^{n-1}\left(u_{(1)}^{\prime}\right) \otimes \delta_{t}^{1}\left(u_{(2)}^{\prime}\right)$, which is an element of $h^{n} \mathfrak{J}^{\otimes n}$ thanks to the induction hypothesis.

By the above we have proved the inclusion $\delta_{s}\left(H_{h}\right) \subseteq \delta_{t}\left(H_{h}\right)$; the reverse inclusion can be shown in the same way, so to give $\delta_{s}\left(H_{h}\right)=\delta_{t}\left(H_{h}\right)$. By Theorem 6.2.9 - giving ' $H_{h}=\delta_{t}\left(H_{h}\right)$ - and Theorem 6.2.12 - giving $H_{h}^{\prime}=$ $\delta_{s}\left(H_{h}\right)$ - this eventually implies $H_{h}^{\prime}={ }^{\prime} H_{h}$.

For $H_{h}$ a RQUEAd, we can provide a direct proof by the same arguments used for a LQUEAd; otherwise, we can deduce the result for RQUEAd's from that for LQUEAd's, as follows.

If $H_{h}$ is a RQUEAd, then $\left(H_{h}\right)_{\text {coop }}^{o p}$ is a LQUEAd; then we have the chain of identities $\left(H_{h}^{\prime}\right)_{\text {coop }}^{o p}={ }^{\prime}\left(\left(H_{h}\right)_{\text {coop }}^{o p}\right)=\left(\left(H_{h}\right)_{\text {coop }}^{o p}\right)^{\prime}=\left({ }^{\prime} H_{h}\right)_{\text {coop }}^{o p}$, whence ${ }^{\prime} H_{h}=H_{h}^{\prime}$ follows too.

We are now ready for the main result of this subsection. In short, it claims that the construction $H_{h} \mapsto{ }^{\prime} H_{h}=H_{h}^{\prime}$, starting from a quantization of $L-$ of type $V^{\ell / r}(L)$ - provides a quantization of the dual Lie-Rinehart bialgebra $L^{*}-$ of type $J^{\ell / r}\left(L^{*}\right)$; moreover, this construction is functorial.

Theorem 6.2.15. (a) Let $V^{\ell}(L)_{h} \in(\text { LQUEAd })_{A_{h}}$, where $L$ is a Lie-Rinehart algebra which, as an A-module, is projective of finite type. Then:

(a.1 ${ }^{\prime} V^{\ell}(L)_{h}=V^{\ell}(L)_{h}^{\prime} \in(\mathrm{LQFSAd})_{A_{h}}$, with semiclassical limit $V^{\ell}(L)_{h}^{\prime} / h V^{\ell}(L)_{h}^{\prime}$ $\cong J^{\ell}\left(L^{*}\right)$. Moreover, the structure of Lie-Rinehart bialgebra induced on $L^{*}$ by the quantization $V^{\ell}(L)_{h}^{\prime}$ of $J^{\ell}\left(L^{*}\right)$ is dual to that on $L$ by the quantization $V^{\ell}(L)_{h}$ of $V^{\ell}(L)$; 
(a.2) the definition of $V^{\ell}(L)_{h} \mapsto{ }^{\prime} V^{\ell}(L)_{h}=V^{\ell}(L)_{h}^{\prime}$ extends to morphisms in (LQUEAd), so that we have a well defined (covariant) functor ${ }^{\prime}()=()^{\prime}$ : (LQUEAd) $\longrightarrow$ (LQFSAd).

(b) Let $V^{r}(L)_{h} \in(\operatorname{RQUEAd})_{A_{h}}$, where $L$ is a Lie-Rinehart algebra which, as an A-module, is projective of finite type. Then:

(b.1) ${ }^{\prime} V^{r}(L)_{h}=V^{r}(L)_{h}^{\prime} \in(\mathrm{RQFSAd})_{A_{h}}$, with semiclassical limit $V^{r}(L)_{h}^{\prime} / h V^{r}(L)_{h}^{\prime} \cong J^{r}\left(L^{*}\right)$. Moreover, the structure of Lie-Rinehart bialgebra induced on $L^{*}$ by the quantization $V^{r}(L)_{h}^{\prime}$ of $J^{r}\left(L^{*}\right)$ is dual to that on $L$ by the quantization $V^{r}(L)_{h}$ of $V^{r}(L)$;

(b.2) the definition of $V^{r}(L)_{h} \mapsto{ }^{\prime} V^{r}(L)_{h}=V^{r}(L)_{h}^{\prime}$ extends to morphisms in (RQUEAd), so that we have a well defined (covariant) functor ${ }^{\prime}()=()^{\prime}$ : (RQUEAd) $\longrightarrow$ (RQFSAd).

Proof. (a) Given $V^{\ell}(L)_{h} \in(\text { LQUEAd })_{h}$, we know that $J^{r}(L)_{h}:=V^{\ell}(L)_{h}^{*} \in$ $(\text { RQFSAd })_{A_{h}}$, by Theorem 5.1.5(a); then $V^{r}\left(L^{*}\right)_{h}:=J^{r}(L)_{h}^{\vee} \in(\text { RQUEAd })_{A_{h}}$ is a quantization of $V^{r}\left(L^{*}\right)$, by Theorem 6.1.4. By Proposition 6.2.2, $\left(V^{\ell}(L)_{h}\right)^{\prime}=$ $*\left(J^{r}(L)_{h}^{\vee}\right)$ is a quantization of $J^{\ell}\left(L^{*}\right)$, by Theorem 5.1.5. In all this, $L^{*}$ stands for the $A$-module dual to $L$ endowed with the Lie-Rinehart bialgebra structure dual to that defined on $L$ by the quantization $V^{\ell}(L)_{h}-$ according to Theorem 4.1.3. This completes the proof of (a.1).

As to (a.2), let $H_{h}=V^{\ell}\left(L_{A}\right)_{h}$ be a LQUEAd over $A_{h}$ and $\Gamma_{h}=V^{\ell}\left(L_{B}\right)_{h}$ a LQUEAd over $B_{h}$, and let $\phi:=(f, F)$ be a morphism of left bialgebroids among them. Set $\mathfrak{J}_{H_{h}}:=\operatorname{Ker}\left(\epsilon_{H_{h}}\right)$ and $\mathfrak{J}_{\Gamma_{h}}:=\operatorname{Ker}\left(\epsilon_{\Gamma_{h}}\right)$. Then $F\left(\mathfrak{J}_{H_{h}}\right) \subseteq \mathfrak{J}_{\Gamma_{h}}$ by the property $\epsilon_{\Gamma_{h}} \circ F=f \circ \epsilon_{H_{h}}$ of a morphism of bialgebroids. Similarly, one has $F^{\otimes n} \circ \Delta_{H_{h}}^{n}=\Delta_{\Gamma_{h}}^{\otimes n} \circ F$ and $F \circ s_{H_{h}}^{\ell}=s_{\Gamma_{h}}^{\ell}$; from this, one easily sees that $\delta_{s}^{n}(F(u))=F^{\otimes n}\left(\delta_{s}^{n}(u)\right)$. From all this we get $F\left(H_{h}^{\prime}\right) \subseteq \Gamma_{h}^{\prime}$, so the restriction of the morphism $(f, F)$ between $H_{h}$ and $\Gamma_{h}$ provides a morphism in (LQFSAd) between $H_{h}^{\prime}$ and $\Gamma_{h}^{\prime}$.

(b) A direct proof for claim (b) can be given by the same arguments used for (a). Otherwise, we can deduce (b) from (a) as follows.

If $H_{h} \in\left(\right.$ RQUEAd), then $\left(H_{h}\right)_{\text {coop }}^{o p} \in\left(\right.$ LQUEAd) and $\left(\left(H_{h}\right)_{\text {coop }}^{o p}\right)^{\prime}=\left({ }^{\prime} H_{h}\right)_{\text {coop }}^{o p}$, so that $H_{h}^{\prime}={ }^{\prime} H_{h}=\left(\left(\left(H_{h}\right)_{\text {coop }}^{o p}\right)^{\prime}\right)_{\text {coop }}^{\text {op }}$. From this we can easily deduce claim (b) from claim (a).

6.2.16. Description of $V^{\ell}(L)_{h}^{\prime}$ when $L$ is a (finite type) free $A$-module. Let $L$ be a Lie-Rinehart algebra which, as an $A$-module, is free of finite type. Let $V^{\ell}(L)_{h} \in$ (LQUEAd) $A_{h}$ be a quantization of $V^{\ell}(L)$; by the freeness of $L$, we can provide an explicit description of $V^{\ell}(L)_{h}^{\prime}$, much like that given in [12] for the similar case of quantum universal enveloping algebras.

First of all, consider $K_{h}:=V^{\ell}(L)_{h}^{*} \equiv J^{r}(L)_{h} \in(\operatorname{RQFSAd})_{A_{h}}$, which (cf. Theorem 5.1.5) is a quantization of $J^{r}(L)$. From Proposition 6.2.2 we have $V^{\ell}(L)_{h}^{\prime} \cong *\left(K_{h}^{\vee}\right)$. 
Let $\left\{\bar{e}_{i}\right\}_{i \in\{1, \ldots, n\}}$ be a basis of the free $A$-module $L$. Then (by the PoincaréBirkhoff-Witt theorem) the set of ordered monomials $\left\{\bar{e}^{\underline{\alpha}}\right\}_{\underline{\alpha} \in \mathbb{N}^{n}}$ is an $A$-basis of $V^{\ell}(L)$, where $\bar{e}^{\underline{\alpha}}:=\bar{e}_{1}^{\alpha_{1}} \cdots \bar{e}_{n}^{\alpha_{n}}$.

Let $\bar{\xi}_{i} \in \operatorname{Hom}\left(V^{\ell}(L), A\right) \cong \widehat{S_{A}\left(L^{*}\right)}$ be defined by $\left\langle\bar{\xi}_{i}, \bar{e}_{1}^{\alpha}\right\rangle=\delta_{\alpha_{1}, 0} \cdots \delta_{\alpha_{i}, 1} \cdots$ $\delta_{\alpha_{n}, 0}$. Then the ordered monomials $\frac{1}{\alpha !} \xi^{\underline{\alpha}}$ (with $\underline{\alpha} !:=\alpha_{1} ! \cdots \alpha_{n} !$ ) is a pseudobasis i.e., a basis in topological sense - of the $A$-module $J^{r}(L)$ dual to the PBW basis $\left\{\bar{e}^{\underline{\alpha}}\right\}_{\underline{\alpha} \in \mathbb{N}^{n}}$.

Lift $\left\{\bar{\xi}_{i}\right\}_{i \in\{1, \ldots, n\}}$ to a subset $\left\{\xi_{i}\right\}_{i \in\{1, \ldots, n\}}$ in $J^{r}(L)_{h}=K_{h}$ such that $\partial_{h}\left(\xi_{i}\right)=0$; then $\left\{\frac{1}{\alpha !} \xi^{\underline{\alpha}}\right\}_{\underline{\alpha} \in \mathbb{N}^{n}}$ is a topological pseudo-basis of $J^{r}(L)_{h}=K_{h}$. Let $\left\{\theta^{\underline{\alpha}}\right\}_{\underline{\alpha} \in \mathbb{N}^{n}}$ be the topological basis of $V^{\ell}(L)_{h}$ dual to $\left\{\frac{1}{\alpha !} \xi^{\underline{\alpha}}\right\}_{\underline{\alpha} \in \mathbb{N}^{n}}$; then $\left\{\theta^{\underline{\alpha}}\right\}_{\underline{\alpha} \in \mathbb{N}^{n}}$ is a lift of the PBW basis $\left\{\bar{e}^{\underline{\alpha}}\right\}_{\underline{\alpha} \in \mathbb{N}^{n}}$ of $V^{\ell}(L)$. Using these tools, a straightforward analysis shows that

$$
V^{\ell}(L)_{h}^{\prime}=\left\{\sum_{\underline{\alpha}} t^{\ell}\left(a_{\underline{\alpha}}\right) h^{|\underline{\alpha}|} \theta_{\underline{\alpha}} \mid a_{\underline{\alpha}} \in A_{h}\right\}
$$

where the summation symbol denotes $h$-adically convergent series.

6.3. Quantum duality for quantum groupoids. We consider now the composition of two Drinfeld's functors. We shall prove that the functors ()$^{\vee}$ and ()$^{\prime}='()$ are actually inverse to each other, so that they establish equivalences of categories $(\mathrm{RQFSAd}) \cong(\mathrm{RQUEAd})$ and $($ LQFSAd $) \cong($ LQUEAd $)$. Our result reads as follows:

\section{Theorem 6.3.1.}

(a) If $K_{h} \in\left(\right.$ RQFSAd), then $\left(K_{h}^{\vee}\right)^{\prime}=K_{h}={ }^{\prime}\left(K_{h}^{\vee}\right)$.

(b) If $K_{h} \in\left(\right.$ LQFSAd), then $\left(K_{h}^{\vee}\right)^{\prime}=K_{h}={ }^{\prime}\left(K_{h}^{\vee}\right)$.

(c) If $H_{h} \in\left(\right.$ LQUEAd), then $\left(H_{h}^{\prime}\right)^{\vee}=H_{h}=\left({ }^{\prime} H_{h}\right)^{\vee}$.

(d) If $H_{h} \in\left(\right.$ RQUEAd), then $\left(H_{h}^{\prime}\right)^{\vee}=H_{h}=\left({ }^{\prime} H_{h}\right)^{\vee}$.

(e) The functors ()$^{\vee}:($ RQFSAd $) \rightarrow\left(\right.$ RQUEAd) and ()$^{\prime}={ }^{\prime}():($ RQUEAd $) \rightarrow$ (RQFSAd) are inverse to each other, hence they are equivalences of categories. Similarly for the functors ()$^{\vee}:$ (LQFSAd) $\longrightarrow$ (LQUEAd) and ()$^{\prime}={ }^{\prime}():($ LQUEAd $) \longrightarrow($ LQFSAd).

Proof. Clearly, claim (e) is just a consequence of the previous items in the statement. We begin by focusing on claim (a): we assume that $K_{h} \in\left(\right.$ RQFSAd) $A_{h}$ and we shall prove that $\left(K_{h}^{\vee}\right)^{\prime}=K_{h}$.

Let us show that $K_{h} \subseteq\left(K_{h}^{\vee}\right)^{\prime}$.

Given $\lambda$ is in $K_{h}$, consider its $n$-th iterated coproduct $\Delta^{n}(\lambda)=\lambda_{(1)} \otimes \cdots \otimes \lambda_{(n)}$; if we write every $\lambda_{(i)}$ as $\lambda_{(i)}=\lambda_{(i)}^{\prime}+\lambda_{(i)}^{\prime \prime}$ with $\lambda_{(i)}^{\prime}:=\lambda_{(i)}-s_{h}^{r}\left(\partial\left(\lambda_{(i)}\right)\right) \in \mathfrak{J}_{h}:=$ $\operatorname{Ker}\left(\partial_{K_{h}}\right)$ and $\lambda_{(i)}^{\prime \prime}:=s_{h}^{r}\left(\partial\left(\lambda_{(i)}\right)\right) \in s_{h}^{r}\left(A_{h}\right)$, then expanding again $\Delta^{n}(\lambda)$ we can 
write it as a sum $\Delta^{n}(\lambda)=\sum \lambda_{(1)}^{\circ} \otimes \cdots \otimes \lambda_{(n)}^{\circ}$ in which $\lambda_{i}^{\circ} \in \mathfrak{J}_{h}$ or $\lambda_{i}^{\circ} \in s_{h}^{r}\left(A_{h}\right)$ for every $i=1, \ldots, n$.

Now let $\alpha_{1}, \ldots, \alpha_{n} \in I_{*}\left(K_{h}^{\vee}\right):=\epsilon_{*}^{-1}\left(K_{h}^{\vee}\right)\left(h A_{h}\right)$. As every $\alpha_{j}$ belongs to $\left(K_{h}^{\vee}\right)$, it defines a map from $\mathfrak{J}_{h}$ to $h A_{h}$. Hence $\left\langle\alpha_{i}, \lambda_{j}\right\rangle \in h A_{h}$ and one has $\left\langle\alpha_{1} \cdots \alpha_{n}, \lambda\right\rangle \in$ $h^{n} A_{h}$. Thus, for any $n \in \mathbb{N}$, we have that $\lambda$ defines a map $\Lambda_{n}: h^{-n} I_{*}^{n}\left(K_{h}^{\vee}\right) \longrightarrow A_{h}$. Clearly all these $\Lambda_{n}$ 's match together to define an element $\Lambda \in\left(\left({ }_{*}\left(K_{h}^{\vee}\right)\right)^{\vee}\right)^{*}=$ $\left(K_{h}^{\vee}\right)^{\prime}$; thus we end up with a natural map $K_{h} \longrightarrow\left(K_{h}^{\vee}\right)^{\prime}(\lambda \mapsto \Lambda)$, which is clearly injective. This yields the inclusion $K_{h} \subseteq\left(K_{h}^{\vee}\right)^{\prime}$.

To prove the converse inclusion $K_{h} \supseteq\left(K_{h}^{\vee}\right)^{\prime}$, one proceeds exactly like in [12] - we leave the details to the reader. Similarly, we leave to the reader the proof of (b), analogous to that of (a).

To prove claim (c), consider $H_{h} \in$ (LQUEAd). We have $K_{h}:=H_{h}^{*} \in$ (RQFSAd), and $H_{h}^{\prime}=*\left(\left(H_{h}^{*}\right)^{\vee}\right)={ }_{*}\left(K_{h}^{\vee}\right)$ by Proposition 6.2.2 (a). Now $\Gamma_{h}:=K_{h}^{\vee} \in$ (RQUEAd) by Theorem 6.1.4, and then ' $\left.\Gamma_{h}=\left({ }_{*} \Gamma_{h}\right)^{\vee}\right)^{*}$ by Proposition 6.2.2 (b), which implies $\left.\star_{\star}\left({ }^{\prime} \Gamma_{h}\right)={ }_{\star}\left(\left({ }_{*} \Gamma_{h}\right)^{\vee}\right)^{*}\right)=\left({ }_{*} \Gamma_{h}\right)^{\vee}$. Altogether — also exploiting claim (a) - this gives

$$
\begin{aligned}
\left.\left(H_{h}^{\prime}\right)^{\vee}={ }_{*}\left(K_{h}^{\vee}\right)\right)^{\vee}=\left({ }_{*} \Gamma_{h}\right)^{\vee}={ }_{\star}\left({ }^{\prime} \Gamma_{h}\right) & ={ }_{\star}\left(\Gamma_{h}^{\prime}\right)= \\
& ={ }_{\star}\left(\left(K_{h}^{\vee}\right)^{\prime}\right)={ }_{\star} K_{h}={ }_{\star}\left(H_{h}^{*}\right)=H_{h}
\end{aligned}
$$

This proves (c), and the proof of (d) is entirely similar again.

\section{An example}

In this last section we apply the main construction of the paper - duality functors and Drinfeld's functors - to a toy model, namely a simple (yet non trivial!) quantum groupoid.

We consider the two dimensional Lie $k$-algebra $\mathfrak{g}=k e_{1} \oplus k e_{2}$ with Lie bracket $\left[e_{1}, e_{2}\right]=e_{1}$. It is known that $\mathfrak{g}^{*}$ is a Poisson manifold: we consider $e_{1}$ and $e_{2}$ as coordinates on $\mathfrak{g}^{*}$, denoting them by $x_{1}$ and $x_{2}$ respectively. The Poisson structure on $\mathfrak{g}^{*}$ is determined by $\left\{x_{1}, x_{2}\right\}=\left[e_{1}, e_{2}\right]=e_{1}$.

Let us introduce the Lie $k[[h]]-$ algebra $\mathfrak{g}_{h}:=k[[h]] e_{1} \oplus k[[h]] e_{2}$ with non-zero Lie bracket $\left[e_{1}, e_{2}\right]_{h}:=h e_{1}$. The $h$-adic completion of the enveloping algebra of $\mathfrak{g}_{h}$, namely $A_{h}:=\widehat{U\left(\mathfrak{g}_{h}\right)}$, is a quantization of the Poisson algebra of polynomial functions on $\mathfrak{g}^{*}$, namely $A=S(\mathfrak{g})$.

We write $\mathcal{D}$ for the ring of polynomial differential operators on $\mathfrak{g}^{*}$, with $\partial_{i}:=\frac{\partial}{\partial x_{i}}, i=1,2$. It is the enveloping algebra of the Lie-Rinehart algebra 
$(S(\mathfrak{g}), \operatorname{Der}(S(\mathfrak{g})), i d)$. We endow it with the standard left algebroid stucture and denote by $\mathcal{D}[[h]]$ the trivial deformation of this structure.

Proposition 7.0.2. Fix notation $\theta_{1}:=x_{1} \partial_{1}$. Then

$$
\mathcal{F}:=\sum_{n=0}^{\infty} \frac{h^{n}}{n ! 2^{n}}\left(\theta_{1} \otimes \partial_{2}-\partial_{2} \otimes \theta_{1}\right)^{n}
$$

is a twistor - cf. Definition 3.2.7 - for $\mathcal{D}[[h]]$.

Proof. It is a straightforward computation.

We will now denote by $\mathcal{D}_{h}$ the twist of $\mathcal{D}[[h]]$ by $\mathcal{F}$. As an algebra, $\mathcal{D}_{h}$ is isomorphic to $\left(S(\mathfrak{g}) \otimes S\left(\mathfrak{g}^{*}\right)\right)[[h]]$. The deformation of $A=S(\mathfrak{g})$ defined by $\mathcal{F}$ is $A_{h}=\widehat{U\left(\mathfrak{g}_{h}\right)}$, the $h$-adic completion of the universal enveloping algebra $U\left(\mathfrak{g}_{h}\right)$ of $\mathfrak{g}_{h}$. The source map $s_{\mathcal{F}}^{\ell}$ (an algebra morphism) is determined by

$$
s_{\mathcal{F}}^{\ell}\left(x_{1}\right)=\sum_{n=0}^{\infty} \frac{1}{n !} \frac{h^{n}}{2^{n}} x_{1} \partial_{2}^{n}, \quad s_{\mathcal{F}}^{\ell}\left(x_{2}\right)=x_{2}-h x_{1} \partial_{1}
$$

The target $t_{\mathcal{F}}^{\ell}$ (an algebra antimorphism) the coproduct $\Delta_{\mathcal{F}}$ and the counit $\epsilon$ are determined by

$$
\begin{gathered}
t_{\mathcal{F}}^{\ell}\left(x_{1}\right)=\sum_{n=0}^{\infty} \frac{(-1)^{n}}{n !} \frac{h^{n}}{2^{n}} x_{1} \partial_{2}^{n}, \quad t_{\mathcal{F}}^{\ell}\left(x_{2}\right)=x_{2}+h x_{1} \partial_{1} \\
\Delta_{\mathcal{F}}(X)=\mathcal{F}^{\#-1}(\Delta(X) \cdot \mathcal{F}), \quad \epsilon\left(x_{1}^{\alpha_{1}} x_{2}^{\alpha_{2}} \partial_{1}^{\beta_{1}} \partial_{2}^{\beta_{2}}\right)=x_{1}^{\alpha_{1}} x_{2}^{\alpha_{2}} \partial_{1}^{\beta_{1}} \partial_{2}^{\beta_{2}}(1)
\end{gathered}
$$

(cf. Theorem 3.2.8). Explicitly, $\mathcal{F}$ can be lifted to an element $\widetilde{\mathcal{F}} \in\left(\mathcal{D} \otimes_{k} \mathcal{D}\right)[[h]]$ defined by

$$
\begin{aligned}
\widetilde{\mathcal{F}} & =\exp \left(\frac{h}{2}\left(\theta_{1} \otimes \frac{\partial}{\partial x_{2}}-\frac{\partial}{\partial x_{2}} \otimes \theta_{1}\right)\right) \\
& =\sum_{n=0}^{\infty} \frac{1}{n !} \frac{h^{n}}{2^{n}}\left(\theta_{1} \otimes \frac{\partial}{\partial x_{2}}-\frac{\partial}{\partial x_{2}} \otimes \theta_{1}\right)^{n} \in \mathcal{D} \otimes_{k} \mathcal{D}[[h]]
\end{aligned}
$$

this element $\widetilde{\mathcal{F}}$ is invertible in $\left(\mathcal{D} \otimes_{k} \mathcal{D}\right)[[h]]$ and one has

$$
\widetilde{\mathcal{F}}^{-1}=\exp \left(-\frac{h}{2}\left(\theta_{1} \otimes \frac{\partial}{\partial x_{2}}-\frac{\partial}{\partial x_{2}} \otimes \theta_{1}\right)\right) \in\left(\mathcal{D} \otimes_{k} \mathcal{D}\right)[[h]]
$$

In turn, the element $\widetilde{\mathcal{F}}^{-1}$ defines an element $\mathcal{G} \in \mathcal{D}[[h]] \widehat{\otimes}_{A_{\mathcal{F}}} \mathcal{D}[[h]]$, namely

$$
\mathcal{G}=\sum_{n=0}^{\infty}(-1)^{n} \frac{h^{n}}{2^{n} n !}\left(\theta_{1} \otimes \frac{\partial}{\partial x_{2}}-\frac{\partial}{\partial x_{2}} \otimes \theta_{1}\right)^{n} \in \mathcal{D}[[h]] \widehat{\otimes}_{A_{\mathcal{F}}} \mathcal{D}[[h]]
$$


Now the map

$$
\mathcal{F}^{\#}: \mathcal{D}[[h]] \widehat{\otimes}_{A_{\mathcal{F}}} \mathcal{D}[[h]] \longrightarrow \mathcal{D}[[h]] \widehat{\otimes}_{A} \mathcal{D}[[h]], \quad h_{1} \otimes h_{2} \mapsto \mathcal{F} \cdot\left(h_{1} \otimes h_{2}\right)
$$

is indeed invertible, its inverse being

$\mathcal{F}^{\#-1}: \mathcal{D}[[h]] \widehat{\otimes}_{A} \mathcal{D}[[h]] \longrightarrow \mathcal{D}[[h]] \widehat{\otimes}_{A_{\mathcal{F}}} \mathcal{D}[[h]], \quad h_{1} \otimes h_{2} \mapsto \mathcal{G} \cdot\left(h_{1} \otimes h_{2}\right)$

We will compute now the dual bialgebroids $\left(\mathcal{D}_{h}\right)_{*}$ and $\left(\mathcal{D}_{h}\right)^{*}$.

Computation of $\left(\mathcal{D}_{h}\right)_{*}$. We shall use the isomorphism

$$
\left(\mathcal{D}_{h}\right)_{*} \longrightarrow \operatorname{Hom}(\mathcal{D}, A)[[h]], \quad \lambda \mapsto\left(\partial_{1}^{a} \partial_{2}^{b} \mapsto\left\langle\lambda, \partial_{1}^{a} \partial_{2}^{b}\right\rangle\right)
$$

Let $d e_{1}, d e_{2} \in\left(\mathcal{D}_{h}\right)_{*}$ be such that

$$
\left\langle d e_{1}, \partial_{1}^{a} \partial_{2}^{b}\right\rangle=\delta_{1, a} \delta_{0, b}, \quad\left\langle d e_{2}, \partial_{1}^{a} \partial_{2}^{b}\right\rangle=\delta_{0, a} \delta_{1, b} .
$$

Similarly, let $e_{1}, e_{2} \in\left(\mathcal{D}_{h}\right)_{*}$ be such that

$$
\left\langle e_{1}, \partial_{1}^{a} \partial_{2}^{b}\right\rangle=x_{1} \delta_{0, a} \delta_{0, b}, \quad\left\langle e_{2}, \partial_{1}^{a} \partial_{2}^{b}\right\rangle=x_{2} \delta_{0, a} \delta_{0, b} .
$$

A direct computation shows that

$$
\left\langle d e_{1} \cdot h d e_{2}, \partial_{1}^{a} \partial_{2}^{b}\right\rangle=\left\{\begin{array}{rcccc}
0 & \text { if } & a \geq 2 & \text { or } & b \geq 2 \\
1 & \text { if } & a=1 & \text { and } & b=1 \\
-\frac{h}{2} & \text { if } & a=1 & \text { and } & b=0 \\
0 & \text { if } & a=0 & \text { and } & b=1
\end{array}\right.
$$

Similarly

$$
\left\langle d e_{2}{ }_{h} d e_{1}, \partial_{1}^{a} \partial_{2}^{b}\right\rangle=\left\{\begin{array}{rrrrr}
0 & \text { if } & a \geq 2 & \text { or } & b \geq 2 \\
1 & \text { if } & a=1 & \text { and } & b=1 \\
h & \text { if } & a=1 & \text { and } & b=0 \\
\frac{2}{0} & \text { if } & a=0 & \text { and } & b=1
\end{array}\right.
$$

Hence $d e_{1}{ }_{h} d e_{2}-d e_{2}{ }_{h} d e_{1}=-h d e_{1}$. Set $\check{d e_{i}}:=h^{-1} d e_{i}$. This equality can be written as

$$
\check{d} e_{1} \cdot h \breve{d}_{2}-\check{d} e_{2} \cdot h \check{d}_{1}=-\check{d} e_{1}
$$

Similarly, the following equalities can be established:

$$
\begin{aligned}
& \check{d e}_{1} \cdot h e_{2}-e_{2} \cdot \check{d e_{1}}=-e_{1}, \quad e_{1} \cdot e_{2}-e_{2} \cdot h e_{1}=h e_{1}, \\
& \check{d e}_{1}{ }_{h} e_{1}=e_{1} \cdot h \check{d e}_{1}, \quad \check{d e}_{2} \cdot h e_{2}=e_{2} \cdot h \check{d e}_{2}, \quad \check{d e_{2}} \cdot h e_{1}-e_{1} \cdot h \check{e}_{2}=e_{1}, \\
& s_{*}^{r}\left(e_{i}\right)=e_{i}, \quad t_{*}^{r}\left(e_{i}\right)=e_{i}+h \check{d e}_{i}
\end{aligned}
$$


From the properties of the coproduct, one gets $\Delta\left(e_{i}\right)=1 \otimes e_{i}, \Delta\left(e_{i}+h \check{h e}_{i}\right)=$ $\left(e_{i}+h \tilde{d e_{i}}\right) \otimes 1$, from which we deduce $\Delta\left(d e_{i}\right)=\check{d e_{i}} \otimes 1+1 \otimes d e_{i}$. The coproduct on $\left(\left(\mathcal{D}_{h}\right)_{*}\right)^{\vee}$ is now determined.

Let us also point out the counit of $\left(\left(\mathcal{D}_{h}\right)_{*}\right)^{\vee}$ : it is given by $\partial\left(\check{d e}_{i}\right)=0$ and $\partial\left(e_{i}\right)=e_{i}$.

Remark 7.0.3. Let us introduce the Lie algebra $\mathfrak{g}_{1}$ such that $\mathfrak{g}_{1} \cong \mathfrak{g}=k \check{d e_{1}} \oplus k \check{d e}_{2}$ (as a $k$-vector space) and $[,]_{1}:=-[,]_{\mathfrak{g}}$. Then $\mathfrak{g}_{1}$ acts on $\mathfrak{g}_{h}=k[[h]] e_{1} \oplus k[[h]] e_{2}$ by derivations, via

$$
\mathfrak{g} \longrightarrow \operatorname{Der}\left(\mathfrak{g}_{h}\right), \quad \check{d} e_{1} \mapsto\left\{\begin{array}{l}
e_{1} \mapsto 0 \\
e_{2} \mapsto-e_{1}
\end{array}, \quad \check{d} e_{2} \mapsto\left\{\begin{array}{l}
e_{2} \mapsto 0 \\
e_{1} \mapsto e_{1}
\end{array} .\right.\right.
$$

We may perform the semi direct product $\mathfrak{g}_{1} \ltimes \mathfrak{g}_{h}$ and $\left(\left(\mathcal{D}_{h}\right)_{*}\right)^{\vee}$ is isomorphic to $U\left(\mathfrak{g}_{1} \ltimes \mathfrak{g}_{h}\right)$ as an algebra but not as a bialgebroid.

Let us now compute ${ }^{\prime} \mathcal{D}_{h}$. We proceed in several steps.

- Let us show that $h \partial_{2} \in{ }^{\prime} \mathcal{D}_{h}$.

We shall show that $\left\langle h \partial_{2}, d e_{1}^{a_{1}} d e_{2}^{a_{2}}\right\rangle=0$ if $\left(a_{1}, a_{2}\right) \neq(0,1)$. We have three cases:

First case: $a_{2}=0$. In this case it is obvious that $\left\langle\partial_{2}, d e_{1}^{a_{1}}\right\rangle=0$.

Second case: $a_{2}=1$. In this case we have $\left\langle\partial_{2}, d e_{1}^{a_{1}} d e_{2}\right\rangle=\left\{\begin{array}{lll}0 & \text { if } & a_{1} \neq 0 \\ 1 & \text { if } & a_{1}=0\end{array}\right.$.

Third case: $a_{2}>1$. In this case the summands in $\Delta_{\mathcal{F}}\left(\partial_{2}\right)$ that might bring a non zero contribution to $\left\langle\partial_{2}, d e_{1}^{a_{1}} d e_{2}^{a_{2}}\right\rangle$ are those of the form

$$
\partial_{2}^{a_{2}} \otimes \theta_{1}^{a_{2}^{\prime}+a_{2}^{\prime \prime}} \frac{(-1)^{a_{2}^{\prime \prime}}}{a_{2}^{\prime} ! a_{2}^{\prime \prime} !} \frac{h^{a_{2}-1}}{2^{a_{2}-1}} \text { with } a_{2}^{\prime}+a_{2}^{\prime \prime}=a_{2}-1
$$

but $\quad \sum_{a_{2}^{\prime}+a_{2}^{\prime \prime}=a_{2}-1} \partial_{2}^{a_{2}} \otimes \theta_{1}^{a_{2}^{\prime}+a_{2}^{\prime \prime}} \frac{(-1)^{a_{2}^{\prime \prime}}}{a_{2}^{\prime} ! a_{2}^{\prime \prime} !} \frac{h^{a_{2}-1}}{2^{a_{2}-1}}=0$,

so we find again $\left\langle h \partial_{2}, d e_{1}^{a_{1}} d e_{2}^{a_{2}}\right\rangle=0$.

- Let us show that $h \partial_{1} \in{ }^{\prime} \mathcal{D}_{h}$. We will show that $\left\langle h \partial_{1}, d e_{1}^{a_{1}} d e_{2}^{a_{2}}\right\rangle \in h^{a_{1}+a_{2}} A_{h}$. We start by computing $\left\langle\theta_{1}, d e_{1}^{a_{1}} d e_{2}^{a_{2}}\right\rangle$.

First case: $a_{2}=0$. It is easy to check that $\left\langle\theta_{1},\left(d e_{1}\right)^{a_{1}}\right\rangle=\left\{\begin{array}{ll}x_{1} & \text { if } a_{1}=1 \\ 0 & \text { otherwise }\end{array}\right.$.

Second case: $a_{2} \geq 1$. The summands in $\Delta_{\mathcal{F}}\left(\partial_{2}\right)$ that might bring a non zero 
contribution $\left\langle\theta_{1}, d e_{1}^{a_{1}} d e_{2}^{a_{2}}\right\rangle$ are those of the form

$$
\begin{array}{r}
\partial_{2}^{a_{2}} \otimes \theta_{1}^{a_{2}^{\prime}+a_{2}^{\prime \prime}+1} \frac{(-1)^{a_{2}^{\prime \prime}}}{a_{2}^{\prime} ! a_{2}^{\prime \prime !} !} \frac{h^{a_{2}}}{2^{a_{2}}} \text { with } a_{2}^{\prime}+a_{2}^{\prime \prime}=a_{2} ; \\
\text { but } \sum_{a_{2}^{\prime}+a_{2}^{\prime \prime}=a_{2}} \partial_{2}^{a_{2}} \otimes \theta_{1}^{a_{2}^{\prime}+a_{2}^{\prime \prime}+1} \frac{(-1)^{a_{2}^{\prime \prime}}}{a_{2}^{\prime} ! a_{2}^{\prime \prime !} !} \frac{h^{a_{2}}}{2^{a_{2}}}=0,
\end{array}
$$

hence in the end $\left\langle\theta_{1},\left(d e_{1}\right)^{a_{1}}\left(d e_{2}\right)^{a_{2}}\right\rangle=0$.

In conclusion, we find $\left\langle\theta_{1},\left(d e_{1}\right)^{a_{1}}\left(d e_{2}\right)^{a_{2}}\right\rangle=\left\{\begin{array}{ll}x_{1} & \text { if } \quad\left(a_{1}, a_{2}\right)=(1,0) \\ 0 & \text { otherwise }\end{array}\right.$.

Let us now compute $\left\langle\partial_{1}, d e_{1}^{a_{1}} d e_{2}^{a_{2}}\right\rangle$. Again we have several cases to consider.

First case: $a_{2}=0$. It is easy to check that $\left\langle\partial_{1},\left(d e_{1}\right)^{a_{1}}\right\rangle=\left\{\begin{array}{ll}1 & \text { if } a_{1}=1 \\ 0 & \text { otherwise }\end{array}\right.$.

Second case: $a_{2} \geq 1$. In this case one has

$$
\begin{array}{r}
0=\left\langle\theta_{1},\left(d e_{1}\right)^{a_{1}}\left(d e_{2}\right)^{a_{2}}\right\rangle=\left\langle s_{\mathcal{F}}^{\ell}\left(x_{1}\right) \partial_{1}-\sum_{n=1}^{+\infty} \theta_{1} \frac{h^{n}}{2^{n} n !} \partial_{2}^{n},\left(d e_{1}\right)^{a_{1}}\left(d e_{2}\right)^{a_{2}}\right\rangle \\
=x_{1}\left\langle\partial_{1},\left(d e_{1}\right)^{a_{1}}\left(d e_{2}\right)^{a_{2}}\right\rangle-\sum_{n=1}^{a_{2}-1}\left\langle\theta_{1} \frac{h^{n}}{2^{n} n !} \partial_{2}^{n},\left(d e_{1}\right)^{a_{1}}\left(d e_{2}\right)^{a_{2}}\right\rangle \\
+\left\langle\theta_{1} \frac{h^{a_{2}}}{2^{a_{2}} a_{2} !} \partial_{2}^{a_{2}},\left(d e_{1}\right)^{a_{1}}\left(d e_{2}\right)^{a_{2}}\right\rangle .
\end{array}
$$

For $1 \leq n \leq a_{2}-1$, the unique summands in $\Delta_{\mathcal{F}}\left(\theta_{1} \partial_{2}^{n}\right)$ that may bring a non zero contribution to $\left\langle\theta_{1} \partial_{2}^{n},\left(d e_{1}\right)^{a_{1}}\left(d e_{2}\right)^{a_{2}}\right\rangle$ are those of the form

$$
\begin{gathered}
\partial_{2}^{a_{2}} \otimes \theta_{1}^{a_{2}-n+1} \frac{h^{a_{2}-n}}{2^{a_{2}-n}} \frac{(-1)^{c_{2}}}{c_{1} ! c_{2} !} \quad \text { with } c_{1}+c_{2}=a_{2}-n ; \\
\text { but } \sum_{c_{1}+c_{2}=a_{2}-n} \partial_{2}^{a_{2}} \otimes \theta_{1}^{a_{2}-n+1} \frac{h^{a_{2}-n}}{2^{a_{2}-n}} \frac{(-1)^{c_{2}}}{c_{1} ! c_{2} !}=0,
\end{gathered}
$$

hence $\sum_{n=1}^{a_{2}-1}\left\langle\theta_{1} \frac{h^{n}}{2^{n} n !} \partial_{2}^{n},\left(d e_{1}\right)^{a_{1}}\left(d e_{2}\right)^{a_{2}}\right\rangle=0$.

Finally, we remark that $\left\langle\theta_{1} \partial_{2}^{a_{2}},\left(d e_{1}\right)^{a_{1}}\left(d e_{2}\right)^{a_{2}}\right\rangle$ is zero if $a_{1} \neq 1$. Hence, in any case, we have $\left\langle\theta_{1} \partial_{2}^{a_{2}} \frac{h^{a_{2}}}{2^{a_{2}} a_{2} !},\left(d e_{1}\right)^{a_{1}}\left(d e_{2}\right)^{a_{2}}\right\rangle \in h^{a_{1}+a_{2}-1} A_{h}$.

In conclusion, we find that in all cases one has $\left\langle\partial_{1},\left(d e_{1}\right)^{a_{1}}\left(d e_{2}\right)^{a_{2}}\right\rangle \in h^{a_{1}+a_{2}-1} A_{h}$.

Now denote by $\left\{\eta_{a, b}\right\}_{(a, b) \in \mathbb{N}^{2}}$ the topological basis of $\mathcal{D}_{h}$ dual to the basis

$$
\left\{\frac{d e_{1}^{a}}{a !} \frac{d e_{2}^{b}}{b !}\right\}_{(a, b) \in \mathbb{N}^{2}} .
$$


We know that ${ }^{\prime} \mathcal{D}_{h}=\left\{\sum_{(a, b) \in \mathbb{N}^{2}} s_{\mathfrak{F}}^{\ell}\left(\alpha_{a, b}\right) h^{a+b} \eta_{a, b} \mid \alpha_{a, b} \in A_{h}\right\}$. As $h^{a+b} \eta_{a, b}=$ $h^{a+b} \partial_{1}^{a} \partial_{2}^{b}$ modulo $h^{\prime} \mathcal{D}_{h}$, we have

$$
{ }^{\prime} \mathcal{D}_{h}=\left\{\sum_{(a, b) \in \mathbb{N}^{2}} s_{\mathfrak{F}}^{\ell}\left(\alpha_{a, b}\right) h^{a+b} \partial_{1}^{a} \partial_{2}^{b} \mid \alpha_{a, b} \in A_{h}\right\} .
$$

Computation of $\left(\mathcal{D}_{h}\right)^{*}$. We shall compute $\left(\mathcal{D}_{h}\right)^{*}$, using the isomorphism

$$
\left(\mathcal{D}_{h}\right)^{*} \longrightarrow \operatorname{Hom}(\mathcal{D}, A)[[h]], \quad \lambda \mapsto\left(\partial_{1}^{a} \partial_{2}^{b} \mapsto\left\langle\lambda, \partial_{1}^{a} \partial_{2}^{b}\right\rangle\right)
$$

Let $d e_{1}, d e_{2} \in\left(\mathcal{D}_{h}\right)^{*}$ be such that

$$
\left\langle d e_{1}, \partial_{1}^{a} \partial_{2}^{b}\right\rangle=\delta_{1, a} \delta_{0, b}, \quad\left\langle d e_{2}, \partial_{1}^{a} \partial_{2}^{b}\right\rangle=\delta_{0, a} \delta_{1, b} .
$$

Similarly, let $e_{1}, e_{2} \in\left(\mathcal{D}_{h}\right)^{*}$ be such that

$$
\left\langle e_{1}, \partial_{1}^{a} \partial_{2}^{b}\right\rangle=x_{1} \delta_{0, a} \delta_{0, b}, \quad\left\langle e_{2}, \partial_{1}^{a} \partial_{2}^{b}\right\rangle=x_{2} \delta_{0, a} \delta_{0, b} .
$$

Now set $\check{d e_{i}}:=h^{-1} d e_{i}$ for $i=1,2$. Then the following equalities can be established:

$$
\begin{aligned}
& e_{1} \cdot e_{2}-e_{2} \cdot h e_{1}=-h e_{1}, \quad \check{d} e_{1} \cdot h \breve{d} e_{2}-\check{d} e_{2} \cdot h \breve{d} e_{1}=\check{d} e_{1} \\
& \check{d e}_{1} \cdot h e_{2}-e_{2} \cdot \check{d e}_{1}=e_{1}, \quad \check{e}_{1} \cdot h e_{1}=e_{1} \cdot h \check{d e}_{1} \\
& \check{d e}_{2} \cdot h e_{2}=e_{2} \cdot h \check{d e}_{2}, \quad \check{d e}_{2} \cdot h e_{1}-e_{1} \cdot h \check{e}_{2}=-e_{1}
\end{aligned}
$$

Moreover, source and target are

$$
s_{r}^{*}\left(x_{i}\right)=e_{i}+h \check{d e}_{i}, \quad t_{r}^{*}\left(x_{i}\right)=e_{i} .
$$

From the properties of the coproduct, one has also

$$
\Delta\left(e_{i}\right)=e_{i} \otimes 1, \quad \Delta\left(\check{d}_{i}\right)=\check{d e}_{i} \otimes 1+1 \otimes \check{d e_{i}} .
$$

Finally, the counit of $\left(\left(\mathcal{D}_{h}\right)^{*}\right)^{\vee}$ is given by the formulas

$$
\partial\left(\check{d e}_{i}\right)=0, \quad \partial\left(e_{i}\right)=e_{i}
$$

A right bialgebroid isomorphism $\left(\left(\mathcal{D}_{h}\right)_{*}\right)^{\vee} \cong\left(\left(\mathcal{D}_{h}\right)^{*}\right)^{\vee}$. From the above analysis, one sees that there exists a unique isomorphism of right bialgebroids $\phi:\left(\left(\mathcal{D}_{h}\right)_{*}\right)^{\vee} \longrightarrow\left(\left(\mathcal{D}_{h}\right)^{*}\right)^{\vee}$ determined by $\phi\left(e_{i}\right)=e_{i}+h \check{d e}_{i}$ and $\phi\left(\check{d e_{i}}\right)=-\check{d e_{i}}$. 


\section{References}

[1] R. Almeida, A. Kumpera, Structure produit dans la catégorie des algébroides de Lie, Ann. Acad. Brasil Cienc. 53 (1981), 247-250. Zbl 0484.58033 MR 637370

[2] F. W. Anderson, K. R. Fuller, Rings and categories of modules, Second edition, Graduate Texts in Mathematics 13, Springer-Verlag, New York, 1992. Zbl 0765.16001 MR 1245487

[3] G. Böhm, Integral theory for Hopf algebroids, Algebra Represent. Theory 8 (2005), 563599. Zbl 1137.16037 MR 2199210

[4] G. Böhm, Hopf algebroids, Handbook of Algebra 6, 173-235, Elsevier/North-Holland, Amsterdam, 2009. Zbl 1220.16022 MR 2553659

[5] G. Böhm, K. Szlachányi, Hopf algebroids with bijective antipodes: axioms, integrals, and duals, Journal of Algebra 274 (2004), 708-750. Zbl 1080.16035 MR 2043373

[6] D. Calaque, M. Van den Bergh, Hochschild cohomology and Atiyah classes, Advances in Mathematics 224 (2010), 1839-1889. Zbl 1197.14017 MR 2646112

[7] P. Dazord, D. Sondaz, Variétés de Poisson, algébroides de Lie, Publications Dépt. Math. 88-1/B, Univ. Lyon I, 1988, 1-68. MR 1040867

[8] V. G. Drinfeld, Quantum groups, Proc. Intern. Congress of Math. (Berkeley, 1986), 1987, pp. 798-820. Zbl 0667.16003 MR 934283

[9] P. Etingof, E. Schiffmann, Lectures on quantum groups, Second edition, Lectures in Mathematical Physics, International Press, Somerville, MA, 2002. Zbl 1106.17015 MR 2284964

[10] S. Evens, J-H. Lu, A. Weinstein, Transverse measures, the modular class, and a cohomology pairing for Lie algebroids, Quart. J. Math. Oxford Ser. (2) 50 (1999), 417436. Zbl 0968.58014 MR 1726784

[11] G. L. Fel'dman, Global dimension of rings of differential operators, Trans. of Moscow Mathematical Society 1 (1982), 123-147. Zbl 0484.13020

[12] F. Gavarini, The quantum duality principle, Annales de l'Institut Fourier 53 (2002), 809834. Zbl 1054.17011 MR 1907388

[13] P. J. Higgins, K. C. Mackenzie, Duality for base-changing morphisms of vector bundles, modules, Lie algebroids and Poisson structures, Math. Proc. Camb. Phil. Soc. 114 (1993), 471-488. Zbl 0812.55007 MR 1235995

[14] P. J. Hilton, U. Stammbach, A course in homological algebra, Second edition, Graduate Texts in Mathematics 4, Springer-Verlag, New York, 1997. Zbl 0863.18001 MR 1438546

[15] J. Huebschmann, Poisson cohomology and quantization, J. Reine Angew. Math. 408 (1990), 57-113. Zbl 0699.53037 MR 1058984

[16] J. Huebschmann, Differential Batalin-Vilkovisky algebras arising from twilled LieRinehart algebras, Poisson geometry (Warsaw, 1998), 87-Ú102, Banach Center Publ. 51, Polish Acad. Sci., Warsaw, 2000. Zbl 1015.17023 MR 1764437

[17] L. Kadison, K. Szlachányi, Bialgebroid actions on depth two extensions and duality, Advances in Mathematics 179 (2003), 75-121. Zbl 1049.16022 MR 2004729 
[18] C. Kassel, V. Turaev, Biquantization of Lie algebras, Pacific Journal of Mathematics 195 (2000), 297-369. Zbl 1040.17008 MR 1782170

[19] M. Khalkhali, B. Rangipour, A new cyclic module Hopf algebras, K-theory 27 (2002), 111-131. Zbl 1020.16029 MR 1942182

[20] Y. Kosmann-Schwarzbach, Exact Gerstenhaber algebras and Lie bialgebroids, Acta Applicandae Mathematicae 41 (1995), 153-165. Zbl 0837.17014 MR 1362125

[21] Y. Kosmann-Schwarzbach, F. Magri, Poisson-Nijenhuis structures, Ann. Inst. H. Poincaré Phys. Théor. 53 (1990), 35-81. Zbl 0707.58048 MR 1077465

[22] N. Kowalzig, Hopf algebroids and their cyclic theory, Ph. D. Thesis. in Mathematics, Universiteit Utrecht, 2009.

[23] N. Kowalzig, U. Krähmer, Duality and products in algebraic (co)homology theories, J. Algebra 323 (2010), no. 1, 2063-2081. Zbl 1268.16008 MR 2594665

[24] N. Kowalzig, H. Posthuma, The cyclic theory of Hopf algebrois, Journal of Noncommutative Geometry 5 (2011), no. 3, 423-476. Zbl 1262.16030 MR 2817646

[25] J.-H. Lu, Hopf algebroids and quantum groupoids, International J. Math. 7 (1996), 4770. Zbl 0884.17010 MR 1369905

[26] K. Mackenzie, P. Xu, Lie bialgebroids and Poisson groupoids, Duke Mathematical Journal 73 (1994), 415-452. Zbl 0844.22005 MR 1262213

[27] S. Montgomery, Hopf algebra and their actions on rings, CBMS Regional Conf. Ser. in Math. 82, American mathematical Society, Providence, RI, 1993. Zbl 0793.16029 MR 1243637

[28] I. Moerdijk, J. Mrčun, On the universal enveloping algebra of a Lie-Rinehart algebra, Proc. Amer. Math. Soc. 138 (2010), 3135-3145. Zbl 1241.17014 MR 2653938

[29] R. Nest, B. Tsygan, Deformations of symplectic Lie algebroids, deformations of holomorphic symplectic structures, and index theorem, Asian J. Mathematics 5 (2001), 599-635. Zbl 1023.53060 MR 1913813

[30] G. Rinehart, Differential forms for general commutative algebras, Trans. American Mathematical Society 108 (1963), 195-222. Zbl 0113.26204 MR 154906

[31] P. Schauenburg, Duals and doubles of quantum groupoids ( $X_{R}-$ Hopf algebras $)$, New trends in Hopf algebra theory (La Falda, 1999), Contemp. Math. 267, Amer. Math. Soc., Providence, RI, 2000, pp. 273-299. Zbl 0974.16036 MR 1800718

[32] M. Takeuchi, Groups of algebras over $A \otimes \bar{A}$, Math. Soc. Japan 29 (1977), no. 3, 459-492. MR 506407

[33] P. Xu, Gerstenhaber algebras and BV-algebras in Poisson geometry, Comm. Math. Phys. 200 (1999), 545-560. Zbl 0941.17016 MR 1675117

[34] P. Xu, Quantum groupoids, Comm. Math. Phys. 216 (2001), 539-581. Zbl 0986.17003 MR 1815717

Received 27 November, 2012; revised 09 July, 2013 
S. Chemla, UPMC Université Paris 06, UMR 7586, Institut de mathématiques de Jussieu, F-75005 Paris, France

E-mail: schemla@math.jussieu.fr

F. Gavarini, Dipartimento di Matematica, Università di Roma "Tor Vergata", via della ricerca scientifica, I-00133 Roma, Italy

E-mail: gavarini@mat.uniroma2.it 